\title{
Random Focusing of Tsunami Waves
}

\author{
Dissertation
}

for the award of

"Doctor of Philosophy"

Division of Mathematics and Natural Sciences

of the Georg-August-Universität Göttingen

within the doctoral programm GGNB (IMPRS)

of the Georg-August-University School of Science (GAUSS)

submitted by

\section{Henri-Philippe Degueldre}

from Courbevoie (France)

Göttingen 2015 


\section{Thesis Committee}

\section{Theo Geisel}

Georg-August University Göttingen

Institute for Nonlinear Dynamics

and

Max-Planck Institute for Dynamics and Self-Organization

Departement of Nonlinear Dynamics

\section{Ragnar Fleischmann}

Max-Planck Institute for Dynamics and Self-Organization

Departement of Nonlinear Dynamics

\section{Eberhard Bodenschatz}

Georg-August University Göttingen

Institute for Nonlinear Dynamics

and

Max-Planck Institute for Dynamics and Self-Organization

Laboratory for Fluid Dynamics, Pattern Formation and Biocomplexity

\section{Members of the examination board}

\section{Joerg Enderlein}

Georg-August University Göttingen

Third Institute of Physics

\section{Reiner Kree}

Georg-August University Göttingen

Institute of Theoretical Physics

\section{Ulrich Parlitz}

Georg-August University Göttingen

Third Insitute of Physics

and

Max-Planck Institute for Dynamics and Self-Organization

Biomedical Physics Group

Date of oral examination : 14.10.2015 


\section{Abstract}

Branched flow is a universal phenomenon of random focusing that occurs in wave or particle flows that propagate in weakly scattering, correlated random media. The consecutive effect of small random forces leads to regions of strong focusing which have the appearance of branches and originate from the formation of random caustics. This phenomenon has been experimentally and theoretically studied in various systems, ranging from experimental observations in electronic microdevices on the micrometer scale to theoretical predictions for the propagation of sound waves in the ocean, on the scale of thousands of kilometers.

Reconstructions of the tsunami of March 2011 exhibited strong fluctuations in the tsunami height, associated with a filamentation of the flow, reminiscent of the structures observed for example in electron flows in semiconductor microstructures. This raises the question, to what extent are the same mechanisms at play in these very different physical systems and what impact do they have for tsunami predictions. Developing a theory of random caustics and branching in tsunami waves is the main purpose of this thesis.

We will start by showing that tsunamis indeed exhibit strong focusing even when propagating over a weakly scattering region of the ocean floor. We will therefore develop the stochastic theory for the characteristic length scale on which random caustics appear in the propagations of tsunamis described by ray equations. We then confirm that the focusing regions of tsunami waves follow the scaling predicted by stochastic ray dynamics with respect to the parameters of the bathymetry. We thus show that tsunamis are indeed subject to the phenomenon of branched flow. We will furthermore demonstrate that, due to the fact that already tiny bathymetry fluctuations can be a source of branched flow, bathymetry has a severe impact on the predictability of tsunami heights. Small uncertainties in the knowledge of the ocean's bathymetry can lead to drastically wrong predictions.

Because the ocean floor bathymetry is known to exhibit anisotropies and to be correlated on several length scales due to the various geological processes contributing to its formation, we later extend the general theory of branched flows to systems where the random medium is correlated on more than one single length-scale, both for tsunami waves and Hamiltonian rays, as it is also relevant to many other systems. We calculate how such correlations affect the typical length scale of branching. Our theory is then applicable to a large variety of correlation functions, either anisotropic or isotropic with multiple correlation lengths.

We conclude with a proposal for an experiment which scales a tsunami event down to the size of a tank in a laboratory to study the focusing effect of bathymetry structures. Such a tool could be useful in tsunami studies and forecasting and it would allow us to experimentally verify our theoretical and numerical results on random focusing of tsunami waves. 



\section{Contents}

$\begin{array}{ll}\text { Abstract } & 3\end{array}$

\begin{tabular}{lll}
\hline 1 & Introduction & 7
\end{tabular}

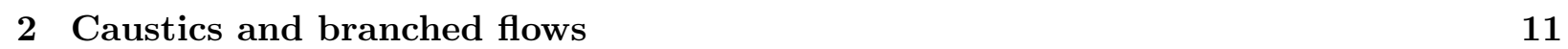

2.1 The phenomenology of branched flow . . . . . . . . . . . . . . . . . . . 11

2.2 Caustics in Lagrangian manifold $\ldots \ldots \ldots \ldots \ldots \ldots$

$2.2 .1 \quad$ Intensity along trajectories $\ldots \ldots \ldots \ldots \ldots \ldots$

2.3 Stability analysis, monodromy and caustics f . . . . . . . . . . . . . 15

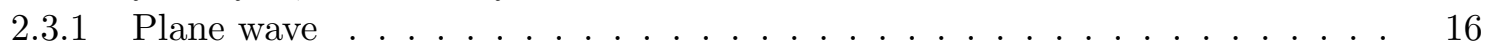

2.3 .2 Point source. . . . . . . . . . . . . . . . . . . . . . . 17

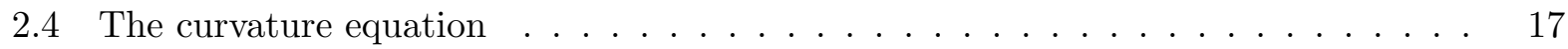

2.5 The quasi-2D approximation $\ldots \ldots \ldots \ldots \ldots \ldots \ldots \ldots$

2.6 The mean distance to the first caustics . . . . . . . . . . . . . . . . . . . . . . . . . . . . . . . .

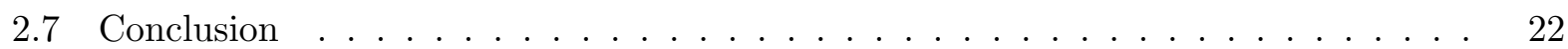

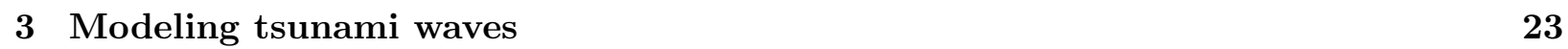

3.1 Phenomenology . . . . . . . . . . . . . . . . . . . . . . . . 23

3.2 The shallow water equations . . . . . . . . . . . . . . . . . . . . . 23

3.3 Dimensionless form and linearization . . . . . . . . . . . . . . . . . . . . 25

3.4 Shallow water rays $\ldots \ldots \ldots \ldots \ldots \ldots \ldots \ldots \ldots$

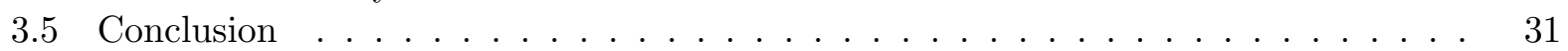

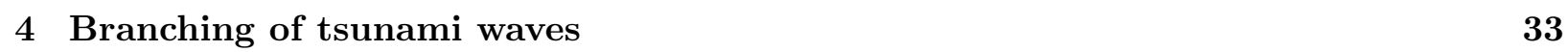

4.1 Branching on a real floor . . . . . . . . . . . . . . . . . . . 33

4.2 Stability analysis and caustics $\ldots \ldots \ldots \ldots \ldots \ldots \ldots \ldots$

4.3 Scaling law . . . . . . . . . . . . . . . . . . . . . . . . . . . . . . 42

4.4 Ray simulations . . . . . . . . . . . . . . . . . . . . . . . . . . . . 45

4.4 .1 Plane waves . . . . . . . . . . . . . . . . . . . . . . . . 46

4.4 .2 Point source . . . . . . . . . . . . . . . . . . . . . . . . 46

4.5 Waves simulations $\ldots \ldots \ldots \ldots \ldots \ldots$

$4.5 .1 \quad$ Scintillation index $\ldots \ldots \ldots \ldots \ldots \ldots$

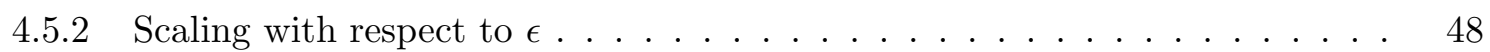

4.6 Impact on tsunami forecasts $\ldots \ldots \ldots \ldots \ldots \ldots$

$4.6 .1 \quad$ Echo-sounding $\ldots \ldots \ldots \ldots \ldots$

4.6 .2 Satellite data . . . . . . . . . . . . . . . . . . . . . . 51

$4.6 .3 \quad$ Inacurrate forecasts due to uncertainties in the bathymetry database . . . . 52

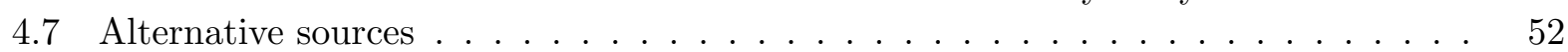

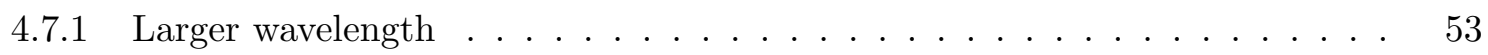

4.7 .2 Fault-like source $\ldots \ldots \ldots \ldots \ldots \ldots \ldots \ldots$

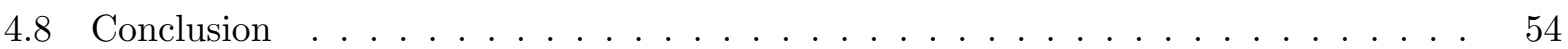




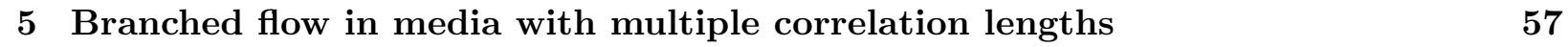

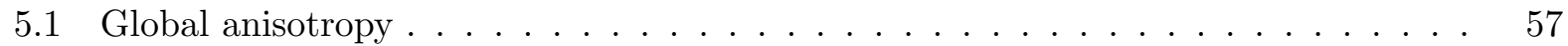

$5.1 .1 \quad$ Classical rays $\ldots \ldots \ldots \ldots \ldots \ldots \ldots$

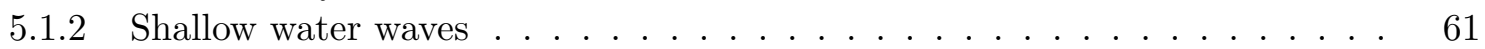

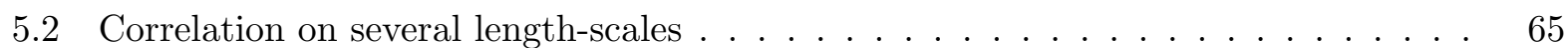

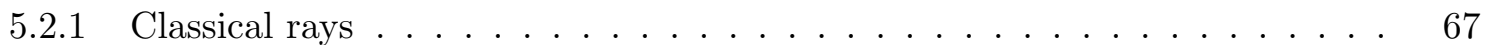

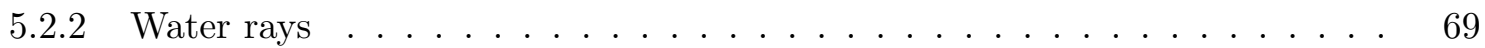

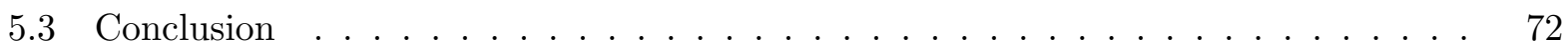

\begin{tabular}{|lll}
\hline & Can we study tsunami focusing in the laboratory? & $\mathbf{7 3}$
\end{tabular}

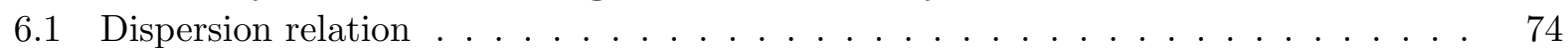

6.2 Surface tension . . . . . . . . . . . . . . . . . . . . . . . . . 74

6.3 Viscosity . . . . . . . . . . . . . . . . . . . . . . . . . . . . 77

6.4 Experimental setup $\ldots \ldots \ldots \ldots \ldots \ldots \ldots \ldots$

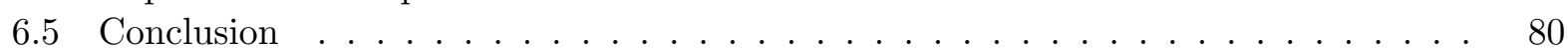

\begin{tabular}{lll}
\hline 7 & Conclusion and outlook & 83
\end{tabular}

\begin{tabular}{|l|l|}
\hline A Modeling the random potentials & 87
\end{tabular}

\begin{tabular}{|lr}
\hline B The Fokker-Planck equation & 89
\end{tabular}

\begin{tabular}{|ll|}
\hline C Modeling a Fault source & 91
\end{tabular}

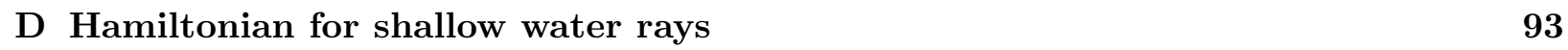

\begin{tabular}{|ll|}
\hline E Remarks on the numerical simulations and open questions & 95
\end{tabular}

$\begin{array}{ll}\text { Acknowledgment } & 101\end{array}$

$\begin{array}{ll}\text { Curriculum Vitae } & 103\end{array}$ 


\section{Chapter 1}

\section{Introduction}

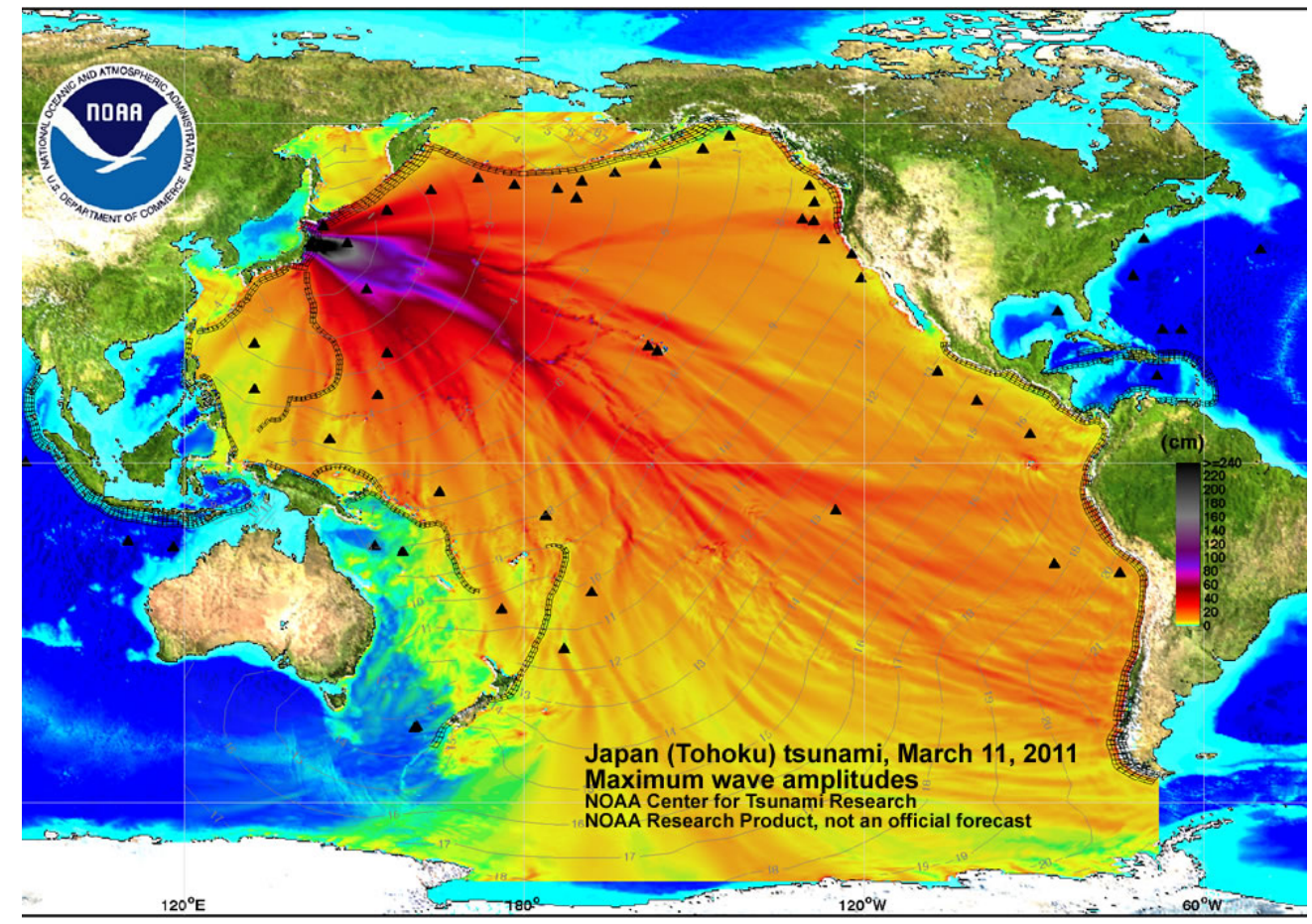

Figure 1.1: This reconstruction of the tsunami induced by the Tohoku earthquake of 2011 shows the maximal wave amplitude during the entire propagation time. We can clearly see that the wave is not propagating homogeneously, but instead shows a filamentation of the flow with high intensity fluctuations, with local amplifications up to four times the local average of the maximal amplitude. source: NOAA

A little more than a decade ago, one of the most destructive natural disasters of our times occurred. On December 26, 2004, the Sumatra-Andaman earthquake produced the deadliest tsunami in recorded history, leading to more than 283,000 deaths [1]. This catastrophic event led the scientific community to an increased effort to improve our understanding of the physics of tsunamis, with many international collaborations, as discussed in reference [2]. Great efforts have been put into the development of tsunami forecasting techniques by improving our models and increasing the accuracy and speed of numerical simulations. One of the main focuses of such studies has been the understanding of the importance of nonlinearity in tsunami physics, especially in order to understand the run-up of waves approaching coasts. However, along most of its propagation path - as long as it propagates over the deep ocean - the tsunami is well-described by linear wave equations. Large libraries of their solutions in the measured topography of the ocean are the basis of planned real time tsunami warning systems. 
A little more than six years later, on March 11, 2011, the Tohoku earthquake, which occurred not far from the coasts of Japan, generated another powerful tsunami which was tremendously destructive, leading to more than 15,000 deaths [3] and the catastrophic events at the Fukushima nuclear power plant. A numerical reconstruction of this tsunami was produced by the National Oceanic and Atmospheric Administration (NOAA) using their forecast tools (MOST model [4] and DART source inference [5]) and is shown in Fig 1.1 .

This plot strongly suggests that some mechanisms leads to correlated multiple focusing events. A single focusing mechanism has been studied by Berry [6] who considered the effect of an isolated large underwater structure such as a volcano on the evolution of tsunami waves in the linear regime. He showed that such underwater structures can focus the wave, in the same fashion as lenses focus light in optics. Furthermore, a recent work by Kanoglu et al. [7] studied the impact of the shape of the source on the propagation of the tsunami wave and it was found that some geometries could indeed be the source of focusing on their own. The complexity of the strong focusing patterns visible in Fig 1.1 , however, suggests that a more elaborate theory is needed to explain it.
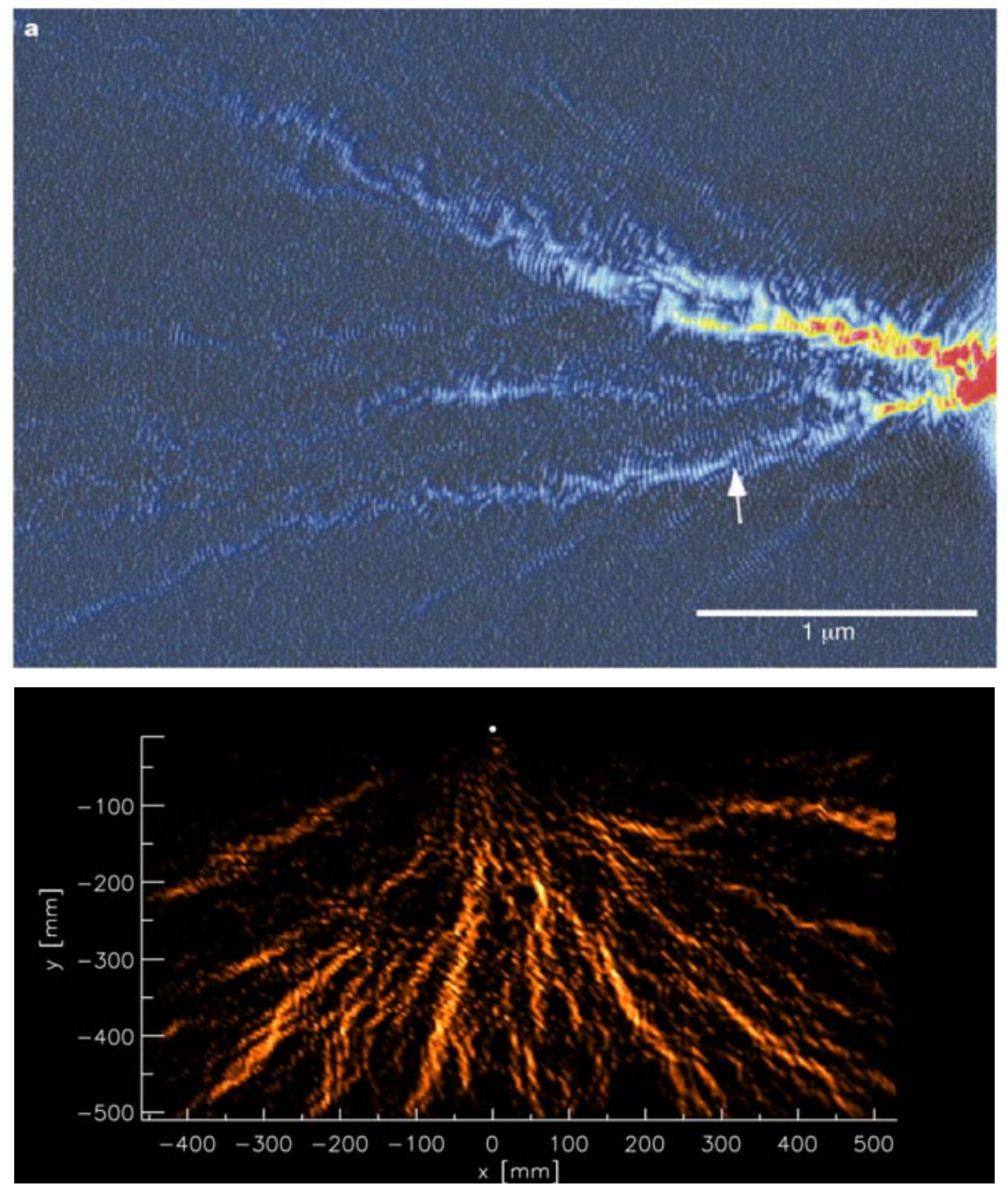

Figure 1.2: TOP - The density of $2 \mathrm{D}$ electron waves emitted by quantum point contact propagating in a semiconductor. The random disorder potential created by donor atoms in the semiconductor is responsible for the branching of the flow. source: [8]. BOTTOM - The intensity of microwaves propagating in a $2 \mathrm{D}$ cavity with a field of random weak scatterers [9]. The flow exhibits high intensity fluctuations in this typical branch-like fashion. source: 10.

Random focusing has been studied for many years and is considered to explain many phenomena, 
including light in optical fibers that is focused by small perturbations 11 and freak waves in the ocean appearing due to small fluctuations in the wave velocity $[12[20]$. The filamentation of the flow that can be observed in Fig 1.1 led us to consider the possibility that tsunami waves could be affected by the phenomenon called branched flow, which was first observed no more than 15 years ago by Topinka et al. 8 in the electron flow of semiconductor heterostructures, as illustrated in the top panel of Fig, 1.2 . The term branched flow has come to describe a general phenomenon that affects particle or wave flows propagating through weakly scattering, correlated random media. In such systems, the consecutive effect of weak scattering events that are correlated in space leads to strong focusing in branch-like patterns, connected to the formation of random caustics, which are regions where the flow is focused. Examples of this phenomenon include the random focusing of electrons in semiconductors [8,21 24] and microwaves propagating in cavities with weak scatterers [9], as shown in Fig.1.2, and it is considered to explain the twinkling of starlight due to its propagation through the slightly inhomogeneous atmosphere [25 29], as well as the focusing of sound waves in the ocean due to water density variations $30-36]$. All those phenomena can be modeled using Schrödinger-type equations, which can then be studied using Hamiltonian rays. This is, however, not the case for shallow water waves. A theory of branched flows of tsunami waves was thus still lacking.

As the phenomenon of branched flow and the emergence of random caustics lead to strong intensity fluctuations (i.e. extreme events), its relevance for tsunami propagation is a topic of great interest and will be the main subject of this thesis, which will be organized as follows:

In the two introductory chapters 2 and 3 , we will recapitulate some of the theoretical foundations of this thesis.

In chapter 2, we will present the theoretical basis of random caustics and branched flow in the framework of two-dimensional Hamiltonian systems. We will focus on the tools that will be used later in this thesis and show the derivations of important results, such as the mean distance to the first caustics, which is the characteristic length scale of the branched flow phenomenon. In chapter 3 , we will derive the linear shallow water equations, which are the relevant model for tsunami propagation in deep ocean conditions, i.e. far from the coasts. Furthermore, as most of the tools developed in chapter 2 use ray equations, since caustics are a classical phenomenon well-defined in the ray picture, we will also derive the associated shallow water ray equations which will be at the center of our theoretical considerations.

In the main part of the thesis, we will extend the theory of branched flow to tsunami waves and derive new general results on random focusing.

Chapter 4 is the heart of this work. Using the tools from chapter 2 and 3, we will answer the questions posed above, whether tsunami waves are affected by branched flow, and discuss its impact on tsunami forecasting. To this aim, we will develop the theory of random caustics for shallow water rays.

So far, all theories of branched flow, including chapter 4 , have characterized the random medium by a single scaled isotropic correlation function. However, the fluctuations of the ocean floor's topography, in most areas, are anisotropic and usually correlated on several length scales, because of the interplay between various geological mechanisms. In chapter 5 , we will therefore answer the following question: what impact does the inclusion of a second correlation length and anisotropy have on the theory of branched flow? We will confirm our results with numerical simulations. We do this not only for the shallow water equations, but for Schrödinger-type wave equations as well, having in mind applications to e.g. the electron transport in semiconductors with anisotropic mass or the scattering by many isolated scatterers as in [9], where the size of the scatterer and the distance between the scatterers are naturally two distinct length scales.

In chapter 6 , we will finally pose the question of whether it is possible to study the focusing of tsunami waves in a laboratory experiment. We give theoretical estimates that lead us to propose 
an experimental setup.

Chapter 7 summarizes all results and gives an outlook, summarizing the new research directions in which the results are pointing. 


\section{Chapter 2}

\section{Caustics and branched flows}

The main purpose of this work is to study the appearance of random focusing in tsunami waves, through a phenomenon called branched flow. This phenomenon has already been studied for classical Newtonian rays in quantum systems (in the semi-classical limit), such as electron propagation in semi-conductors [8, 21, 23] or microwaves scattering [9], and we believe that the best approach is to first lay down the theoretical basis and previous relevant results that influenced the way this research was conducted. The purpose of this section is to introduce the reader to the concept of focusing by random caustics and its impact on wave propagation in disordered media, as well as the methods and tools used in order to quantify it. Previous results that concern quantum systems will be derived here, as they will serve as a reference when studying tsunami waves. Furthermore, some of the analytical tools developed for such systems will be used later on in this work. We will first give a phenomenological description of branched flows in order to establish the framework of this chapter. Then, we will explain what is meant by focusing and how this phenomenon is understood in a ray picture, in term of the curvature of the action and the Hamilton-Jacobi equation, associated with the concept of Lagrangian manifold. Furthermore, we will introduce some elements of stability analysis in order to understand the appearance of focus points and develop an approximation that allows us to treat the system stochastically. Finally, we will use tools from stochastic theory to compute a fundamental quantity in the study of branched flows, which is the mean distance that separates the source from the first caustics. Most of the content of this section was covered in details in [37], therefore this introductory chapter will mainly be a summary of what was done in this previous work.

\subsection{The phenomenology of branched flow}

The phenomenon of branched flow emerges from the appearance of random caustics in wave fields propagating through correlated random media. When waves or particles propagate through a correlated random medium, the random focus points tend to be aligned in space in branch-like patterns, which actually take the geometry of catastrophes known as folds and cusps [38, 39]. As it seems straightforward to understand how caustics might appear in a strongly scattering medium, it is less so when the medium is only weakly scattering, with a potential typically varying on a scale that is on the order of a percent of the energy of the particles. Nevertheless, relatively recent experiments [8,9] showed that even small disorders in the potential can lead to very strong fluctuations in the particle flow intensity. This suggest the idea that the consecutive effects of small focusing events that are correlated in space tend to build up to produce strong intensity fluctuations, which happen to take the shape of branches, as illustrated in Fig 2.1. In this figure, we can also see that the first branches, corresponding to the first caustics, are the most intense, thus the ones we want to know the most about. Furthermore, as was studied in [23, the time to the first caustics is very important in the determination of the universal statistics of the density of branches, which gives us even more motivations to be interested in this quantity. The rest of this chapter will be dedicated to the characterization of these random 


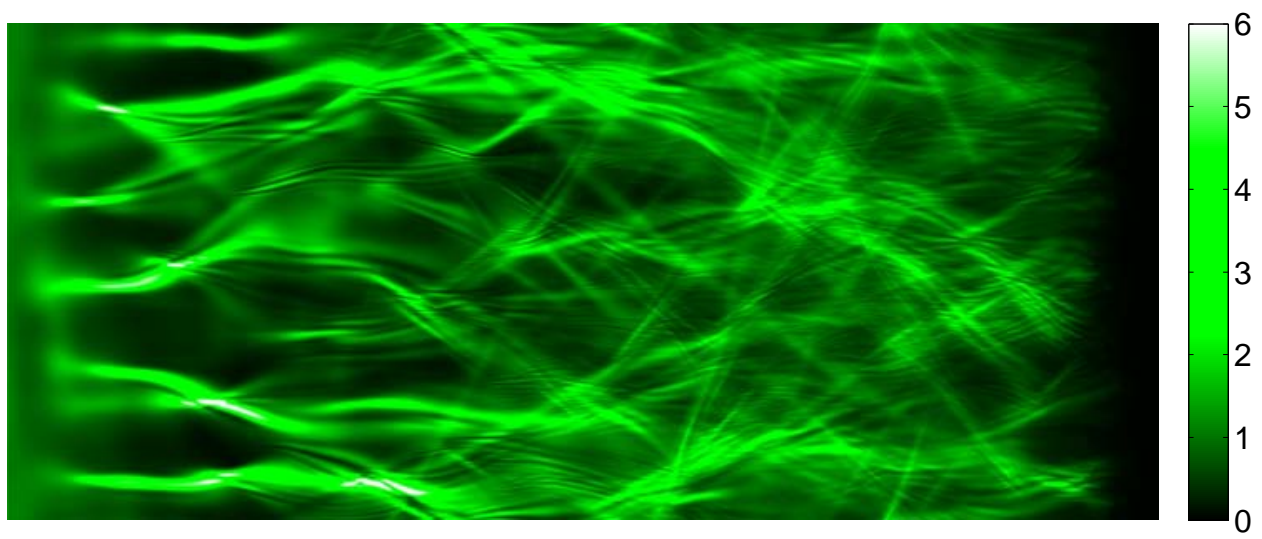

Figure 2.1: An illustration of the intensity of a wave flow propagating from left to right on a random potential (not shown). The intensity has been normalized to the average intensity, meaning that we have wave intensities more than six times higher than the mean (actually, the highest intensity is eleven times higher than the mean, but the color map was cut off at six to highlight the details of the structure of the flow). We see that the strongest intensities appear in the first generation of caustics and branches.

caustics in systems that can be described using Hamiltonian dynamics. Throughout this thesis, we will often refer to such systems in terms of classical rays.

\subsection{Caustics in Lagrangian manifold}

We will be studying classical Hamiltonian systems without interactions, that can be generally described by Hamiltonian functions $\mathcal{H}$ of the form

$$
\mathcal{H}(t, \vec{x}, \vec{p})=\frac{1}{2} \vec{p} \cdot \vec{p}+V(\vec{x}),
$$

where $\vec{x}$ and $\vec{p}$ are the usual space and momentum variables, $V(\vec{x})$ is the static, space-dependent potential, assumed to be smooth, and we set the mass to unity without loss of generality (w.l.o.g). It is well known from classical mechanics that, in such systems, we can write the momentum in terms of the classical action $S$ with

$$
\vec{p}=\frac{\partial S}{\partial \vec{x}},
$$

which allows us to write the Hamilton-Jacobi equation in the following form

$$
\frac{\partial}{\partial t} S(t, \vec{x})+\mathcal{H}\left(t, \vec{x}, \frac{\partial S}{\partial \vec{x}}\right)=0 .
$$

The H-J equation is a first-order nonlinear partial differential equation (PDE) which can be solved by the method of characteristics [40-42]. The characteristics found by this method will correspond to the physical trajectories, which, whenever the system is non-trivial, will eventually cross in position space after a finite time, corresponding to multivalued solutions of the H-J equation. This crossing of the trajectories is at the very origin of the appearance of caustics. Let us consider a ray bundle which spans a $2 n$-dimensional phase space made of all the couples $(\vec{x}, \vec{p})$. Thanks to Eqs. $(2.2)$, which bring $n$ constraints on the phase space, the system is forced onto an $n$-dimensional manifold defined by the couples $(\vec{x}, \vec{p}(\vec{x}))$. This hypersurface is called the Lagrangian manifold $\mathcal{L}$ and satisfies some differential constraints due to its derivation from a scalar in Eqs. 2.2.). 
As the Lagrangian manifold $\mathcal{L}$ is a $n$-dimensional object, it can be parametrized using a new set of $n$ variables $\left(s_{1}, \ldots, s_{n}\right)$, such that $\vec{x}=\vec{x}(\vec{s})$ and $\vec{p}=\vec{p}(\vec{s})$. Furthermore, this description is unique and, as long as the determinant of its Jacobian, det $\left[\frac{\partial x_{i}}{\partial s_{j}}\right]$, is non-zero, the transformation, $\vec{x}=\vec{x}(\vec{s})$, is invertible, such that we can map all the points of the manifold with $(\vec{x}, \vec{p}(\vec{s}(\vec{x}))$. Of course, once we approach a point in $\mathcal{L}$ at which the determinant of the Jacobian is zero, this is no longer the case and the matrix (Einstein's summation convention is implied.)

$$
\frac{\partial p_{i}}{\partial x_{j}}=\frac{\partial p_{i}}{\partial s_{k}} \frac{\partial s_{k}}{\partial x_{j}}
$$

will behave singularly (for $m=1$, it will simply diverge, as $\frac{\partial p}{\partial s}$ cannot be zero at the same time as $\frac{\partial s}{\partial x}$. For $m>2$, however, the exact behavior will depend on the various $\left.\frac{\partial p_{i}}{\partial s_{k}} 43,44\right)$. All the points where $\frac{\partial x_{i}}{\partial s_{k}}=0$ form a set called the singular set, the projection of which onto configuration space corresponds to the location of the caustics. In Fig 2.2, we illustrate this effect in the paraxial approximation, which is presented in details in the section 2.5 about the quasi-2D approximation. For this illustration, it is only needed to know that the paraxial approximation consist in the idea that the propagation direction (here, $x$ ) is assumed to grow linearly in time (so, we neglect the forces in $x$ ) and all the dynamics occurs in the transverse direction $y$. We can see that, at the turning points of the Lagrangian manifold (bottom panel), two rays coalesce and therefore, from here on, the momentum and the action are multivalued.

The condition for the appearance of caustics can also be formulated in terms of the curvature of the action $u_{i j}$, defined by

$$
u_{i j}:=\frac{\partial S}{\partial x_{i} \partial x_{j}}=\frac{\partial p_{i}}{\partial x_{j}},
$$

which corresponds to the same as in Eq. 2.4. This matrix has of course a set of eigenvalues $\left\{\lambda_{i}\right\}$, which are then called the principal curvatures of the action [45]. Whenever one of these eigenvalues becomes infinite, a caustic occurs. We will use the properties of the curvature in order to investigate the appearance of caustics in random potentials at the end of this chapter.

\subsubsection{Intensity along trajectories}

We want to find an expression for the classical intensity of the rays, to show that it diverges at the location of the caustics, which will help us to formulate an analytical condition for a caustic. Let us start with a simple case, with a beam of rays in a plane wave initial condition (propagating in $x$ with an initial momentum $p_{0}$, as we will most often chose in this work), which is parametrized by $x_{k}(0)=0, y_{k}(0)=k, p_{x, k}=p_{0}$ and $p_{y, k}=0$. We will compute the density of the ray beam $\rho(\vec{x}, t)$ at the location $\vec{r}_{k}(t)=\left(x_{k}(t), y_{k}(t)\right)$ along the trajectory with parameter $k$. In order to establish a density, we will first need to define an initial uniform density $\rho_{0}$ in an initial surface element in phase space, which can be defined as $\Delta t \vec{p}_{0} \times \vec{\delta}_{0}$, with $\vec{\delta}_{0}$ a vector along the Lagrangian manifold $\mathcal{L}$ in position space, with length $\delta_{0}$. Of course, this surface element has a weight of $\rho_{0} \Delta t p_{0} \delta_{0}$. The density simply evolves in time and space according to

$$
\rho(t, \vec{x})=\sum_{\text {traj }} \frac{\rho_{0} \Delta t p_{0} \delta_{0}}{|\Delta t \vec{p}(t) \times \vec{\delta}(t, \vec{x})|}=\sum_{\text {traj }} \frac{\rho_{0} p_{0}}{\left|\vec{p}(t) \times \frac{\partial \vec{x}}{\partial k^{\prime}}\right| \frac{\partial k^{\prime}}{\partial k}},
$$

where we sum over all the trajectories which cross the point $(t, \vec{x})$ and $k^{\prime}$ parametrizes the manifold at time t. In the paraxial approximation, where we have $x=t$ and $p_{0}=1$, such that the dynamical direction is $y$, this transforms into

$$
\rho_{\text {paraxial }}=\sum_{\text {traj }} \frac{\rho_{0}}{\partial y k^{\prime}} \frac{\partial k^{\prime}}{\partial k} \text {. }
$$




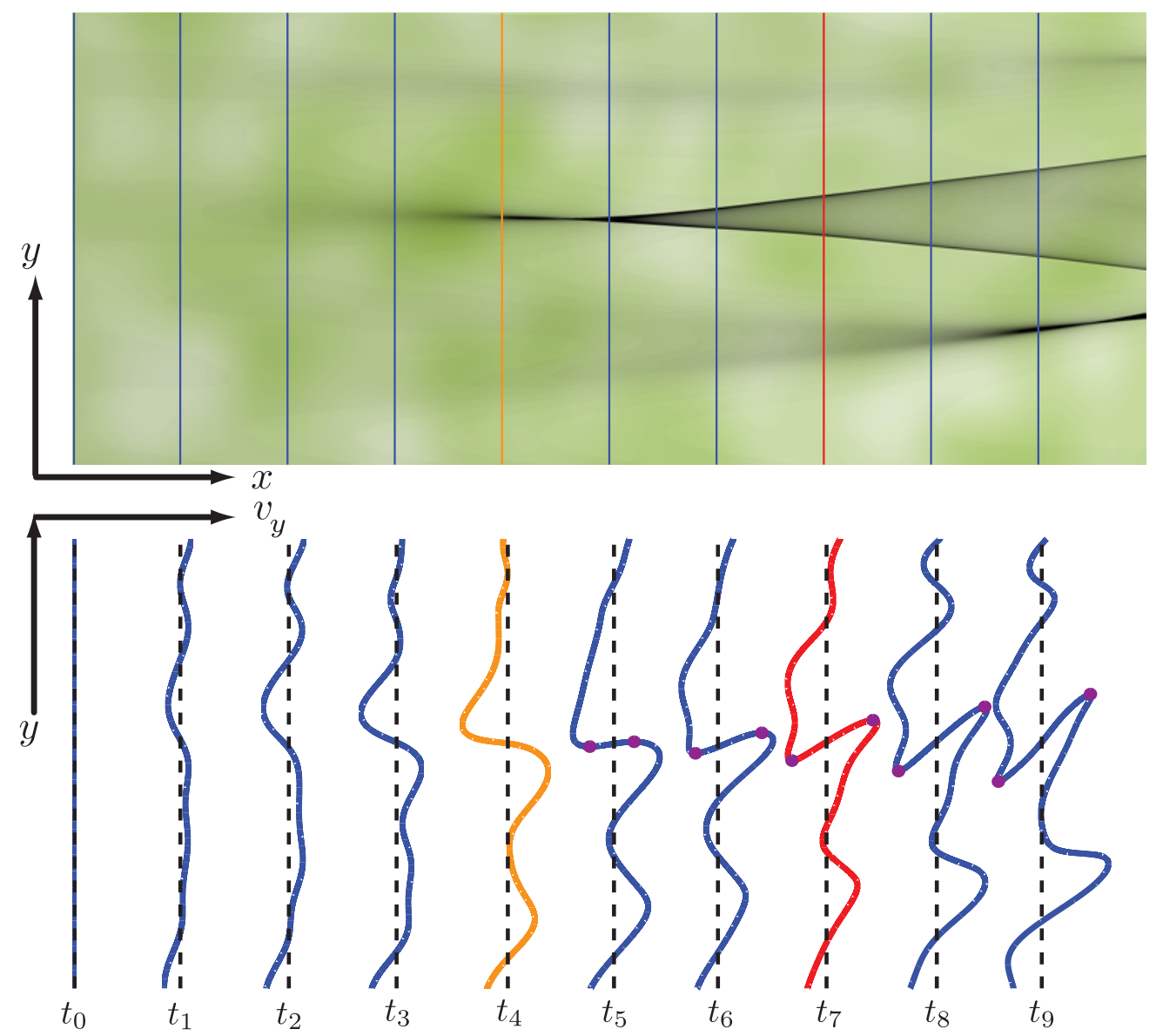

Figure 2.2: The upper panel shows the density (in gray scale) of a ray bundle with initial conditions corresponding to a plane wave flow propagating over a random potential (in yellow-green scale). The bottom panel shows the corresponding Lagrangian manifold. This picture was made in a paraxial approximation, such that the manifold is one-dimensional. We can clearly see how the caustics in the branches correspond to the turning points of the LM (purple). This figure was taken from [37]

This means that a divergence of the density could have two different origins. First, when the projection of the manifold into real space $\frac{\partial y}{\partial k^{\prime}}$ goes to zero. When the stretching of the manifold along the direction of its parametrization $\frac{\partial k^{\prime}}{\partial k}$ goes to zero, it diverges as well. Considering that the length element $\delta k^{\prime}$ can be written as

$$
\delta k^{\prime}=\sqrt{\delta y^{2}+\delta p_{y}^{2}},
$$

we can compute

$$
\frac{\delta k^{\prime}}{\delta y}=\delta y^{-1} \sqrt{\delta y^{2}+\delta p_{y}^{2}}=\sqrt{1+\frac{\delta p_{y}^{2}}{\delta y^{2}}}=\sqrt{1+u^{2}},
$$

where the last equality comes from Eq.2.5 in one dimension (paraxial approximation). We can now insert this into the paraxial density to find

$$
\rho_{\text {paraxial }}=\sum_{\text {traj }} \rho_{0} \frac{\partial k}{\partial k^{\prime}} \sqrt{1+u^{2}} .
$$

This means that, for large curvatures $u$, the density scales linearly in $u$. When the curvature diverges, so does the density. We illustrate the densities of Fig.2.2 at time $t_{4}$ and $t_{7}$ in Fig.2.3. 


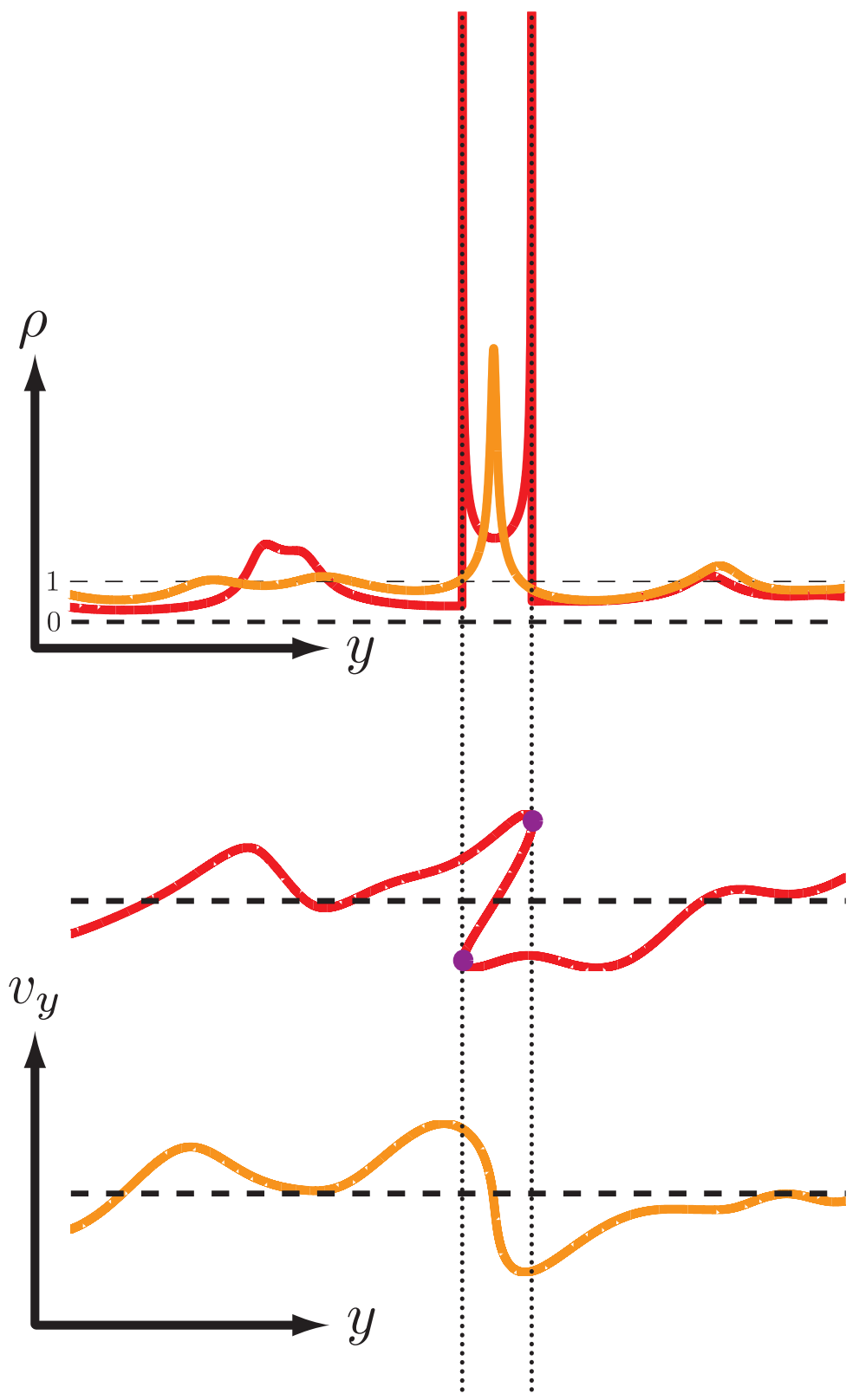

Figure 2.3: The upper panel shows the densities $\rho$, facing their corresponding Lagrangian manifolds in the bottom panel. This picture was also made in the paraxial approximation, such that the manifold is one-dimensional. At the turning points, the density is classically infinite. Between the turning points, we can see that the density is approximately three times the outer value, since the region is covered by three trajectories (three layers of the Lagrangian manifold). This figure was taken from [37]

\subsection{Stability analysis, monodromy and caustics}

The monodromy or stability matrix $M$ is an essential tool in the study of dynamical systems that describes the time evolution of a small phase space volume. In $m$ dimensions, a phase space element $\vec{\phi}=(\vec{x}, \vec{p})$ is a $2 m$-dimensional object, that is simply evolved in time by the $2 m \times 2 m$ stability matrix $M$ with

$$
\vec{\phi}(t)=M(t) \vec{\phi}(0), M(0)=\mathbb{I}_{2 m \times 2 m},
$$


where $\mathbb{I}_{2 m \times 2 m}$ is the identity in $2 m \times 2 m$ dimensions. In Hamiltonian systems, the time evolution of the monodromy matrix is then given by

$$
\dot{M}(t)=\left(\begin{array}{cc}
0 & \mathbb{I}_{2 m} \\
-\mathbb{I}_{2 m} & 0
\end{array}\right)\left(\frac{\partial^{2} \mathcal{H}}{\partial \phi_{i} \partial \phi_{j}}\right) M(t)=: K(t) M(t)
$$

The time evolution of stability matrices without a Hamiltonian will be discussed in section 4.2, where the ray equations cannot be formulated in such terms.

The stability matrix is strongly related to the description of the dynamics of the system and can be associated with the well-known concept of Lyapunov exponents. We can use it to study the appearance of caustics and to compute flow densities by considering a small section of the manifold, as in Eq.(2.6). Indeed, the small section $\vec{\delta}$ corresponds to the projection of the time-evolved $\vec{\delta}_{0}$ onto position space, meaning that we can write it in terms of a projection $\vec{\delta}(t)=P_{\text {pos.sp. }}\left(M(t) \vec{\delta}_{0}\right)$, with the operator $P_{\text {pos.sp. }}$ that projects onto position space. We can then compute the flow density

$$
\rho(t, \vec{x})=\sum_{\text {traj }} \frac{\rho_{0} \Delta t p_{0} \delta_{0}}{|\Delta t \vec{p}(t) \times \vec{\delta}(t, \vec{x})|}=\sum_{\text {traj }} \frac{\rho_{0} p_{0} \delta_{0}}{\vec{p}(t) \times P_{\text {pos.sp. }}\left(M(t) \vec{\delta}_{0}\right)},
$$

where we sum over all the trajectories, as in Eq. 2.6. As we discussed in the previous section, the conditions for a caustic to occur is that the density diverges, which for $m=2$ yields

$$
\vec{p}(t) \times P_{\text {pos.sp. }}\left(M(t) \vec{\delta}_{0}\right)=\left(-p_{x}, p_{y}, 0,0\right)^{T} M \overrightarrow{\delta_{0}}=0 .
$$

This general condition for the appearance of caustics in two dimensions will be very useful, as it allows us to precisely locate the caustics in the fully two dimensional ray simulations. We will now derive specific conditions in the case of plane waves and point sources, which are the two types of initial conditions that we will use throughout this thesis.

We will use $M_{i j}$, which are the elements of the monodromy matrix $M$, to write simple conditions in order to locate the caustics in two dimensions, which we will use in the simulations. The elements have the forms

$$
M_{i j}(t)=\frac{\partial \phi_{i}(t)}{\partial \phi_{j}(0)}
$$

where once again $\phi_{i, j}$ are phase space variables.

\subsubsection{Plane wave}

In Eq.2.14, the only thing we still need to specify is the initial vector $\overrightarrow{\delta_{0}}$. For plane wave initial conditions with $x$ defined as the propagation direction, we have $\delta p_{y}^{0}=0$ and $\delta x^{0}=0$.

Furthermore, we use the constant energy shell condition $H=E$, with $E$ fixed. When we expand the energy in small deviations $\delta \vec{\phi}^{0}=\left(\delta \vec{x}^{0}, \delta \vec{p}^{0}\right)$, it yields

$$
\begin{aligned}
\frac{1}{2}\left(p_{x}^{0}+\delta p_{x}^{0}\right)^{2}+\frac{1}{2}\left(p_{y}^{0}\right)^{2}+V\left(x^{0}, y^{0}+\delta y^{0}\right) \simeq & \frac{1}{2}\left(p_{x}^{0}\right)^{2}+p_{x}^{0} \delta p_{x}^{0}+\frac{1}{2}\left(p_{y}^{0}\right)^{2}+V\left(x^{0}, y^{0}\right) \\
& +V_{y}\left(x^{0}, y^{0}\right) \delta y^{0}+\mathcal{O}\left(\delta \phi_{i}^{0} \delta \phi_{j}^{0}\right) \\
= & \frac{1}{2}\left(p_{x}^{0}\right)^{2}+\frac{1}{2}\left(p_{y}^{0}\right)^{2}+V\left(x^{0}, y^{0}\right)
\end{aligned}
$$

where $V_{y}=\frac{\partial}{\partial y} V$. We can reformulate this equation to get rid of one variable

$$
\delta p_{x}^{0}=-\frac{V_{y}\left(x^{0}, y^{0}\right)}{p_{x}^{0}} \delta y^{0} \equiv \nu_{p w} \delta y^{0} .
$$


This will now be used when we compute the caustic condition Eq. 2.14), with

$$
M \vec{\delta}_{0}=M\left(\begin{array}{c}
0 \\
\delta y^{0} \\
\nu_{p w} \delta y^{0} \\
0
\end{array}\right)=\delta y^{0}\left(\begin{array}{l}
M_{12}+M_{13} \nu_{p w} \\
M_{22}+M_{23} \nu_{p w} \\
M_{32}+M_{33} \nu_{p w} \\
M_{42}+M_{43} \nu_{p w}
\end{array}\right)
$$

we find

$$
\left(-p_{x}, p_{y}, 0,0\right)^{T} M \overrightarrow{\delta_{0}}=-p_{y} \delta y^{0}\left(M_{12}+M_{13} \nu_{p w}\right)+p_{x} \delta y^{0}\left(M_{22}+M_{23} \nu_{p w}\right)=0 .
$$

where we can obviously divide out the $\delta y^{0}$.

\subsubsection{Point source}

We use a very similar derivation for the point source, the only difference lies in the initial conditions, which are now $\delta x^{0}=0$ and $\delta y^{0}=0$ with the fixed energy $H=E$. When we expand the energy in small deviations, it yields

$$
\begin{aligned}
\left(p_{x}^{0}+\delta p_{x}^{0}\right)^{2}+\left(p_{y}^{0}+\delta p_{y}^{0}\right)^{2} & \simeq\left(p_{x}^{0}\right)^{2}+\left(p_{y}^{0}\right)^{2}+2\left(p_{x}^{0} \delta p_{x}^{0}+p_{y}^{0} \delta p_{y}^{0}\right)+\mathcal{O}\left(\delta \phi_{i} \delta \phi_{j}\right) \\
& \Rightarrow \delta p_{x}^{0}=-\frac{p_{y}^{0}}{p_{x}^{0}} \delta p_{y}^{0} \equiv \nu_{p s} \delta y^{0} .
\end{aligned}
$$

This will now be used when we compute the caustic condition Eq.(2.14), with

$$
M \vec{\delta}_{0}=M\left(\begin{array}{c}
0 \\
0 \\
\nu_{p s} \delta p_{y}^{0} \\
\delta p_{y}^{0}
\end{array}\right)=\delta p_{y}^{0}\left(\begin{array}{l}
M_{14}+M_{13} \nu_{p s} \\
M_{24}+M_{23} \nu_{p s} \\
M_{34}+M_{33} \nu_{p s} \\
M_{44}+M_{43} \nu_{p s}
\end{array}\right),
$$

thus it gives

$$
\left(-p_{x}, p_{y}, 0,0\right)^{T} M \overrightarrow{\delta_{0}}=-p_{y} \delta p_{y}^{0}\left(M_{14}+M_{13} \nu_{p s}\right)+p_{x} \delta p_{y}^{0}\left(M_{24}+M_{23} \nu_{p w}\right)=0 .
$$

where, once again, we can divide by $\delta p_{y}^{0}$.

The conditions given by equations Eq. (2.19) and Eq. (2.22) are used in all our numerical simulations for rays in order to find the caustics. Some illustrations are given in chapter 4 .

\subsection{The curvature equation}

While discussing the flow density and its divergence earlier in the chapter (section 2.2.1), we showed that we can link the appearance of caustics with the divergence of the curvature of the action, which was defined as $u_{i j}=\frac{\partial^{2} S}{\partial x_{i} \partial x_{j}}$. As was already mentioned before, the classical action's time evolution satisfies the H-J equation

$$
\frac{\partial}{\partial t} S(\vec{x}, t)+\frac{1}{2}\left(\frac{\partial S}{\partial \vec{x}}\right)^{2}+V(\vec{x})=0
$$

when we consider systems with a simple Hamiltonian as introduced in Eq.(2.1). By taking two spatial derivatives in $x_{i}$ and $x_{j}$ in the previous equation, we can find a PDE for the curvature of the action

$$
\left(\frac{\partial}{\partial t}+p_{k}(t, \vec{x}) \frac{\partial}{\partial x_{k}}\right) u_{i j}(t, \vec{x})+u_{i k}(t, \vec{x}) u_{k j}(t, \vec{x})+\frac{\partial}{\partial x_{i} \partial x_{j}} V(t, \vec{x})=0
$$


where once again the summation convention is implied.

We note that, when considering the characteristic $\frac{d \vec{x}}{d t}=\vec{p}$, which is then simply the velocity field, the term in the brackets can be understood as the convective derivative. This can be changed into a total derivative by changing from an Eulerian to a Lagrangian framework [46, 47]. This implies that, in the Lagrangian view, i.e. along the characteristics, we have

$$
\frac{d}{d t} u_{i j}(t, \vec{x})+u_{i k}(t, \vec{x}) u_{k j}(t, \vec{x})+\frac{\partial}{\partial x_{i} \partial x_{j}} V(t, \vec{x})=0 .
$$

This equation will be the starting point of our analytical study in our effort to compute the mean distance to the first caustics in the case of branched flow, i.e. when studying the propagation of classical rays over a weakly scattering, correlated random potential. The next step is now to reduce the dimensionality of the dynamics by making a set of approximation, which we will call the quasi-2D approximation.

\subsection{The quasi-2D approximation}

As we stated above, our goal is to study Eq. 2.25) in the presence of weak random potentials. By this, we mean that the standard deviation of the potential $\epsilon=\sqrt{\left\langle V^{2}\right\rangle}$, i.e. the typical size of its fluctuations, has to be well below the energy of the particles $E \gg \epsilon$, as illustrated in Fig 2.4.
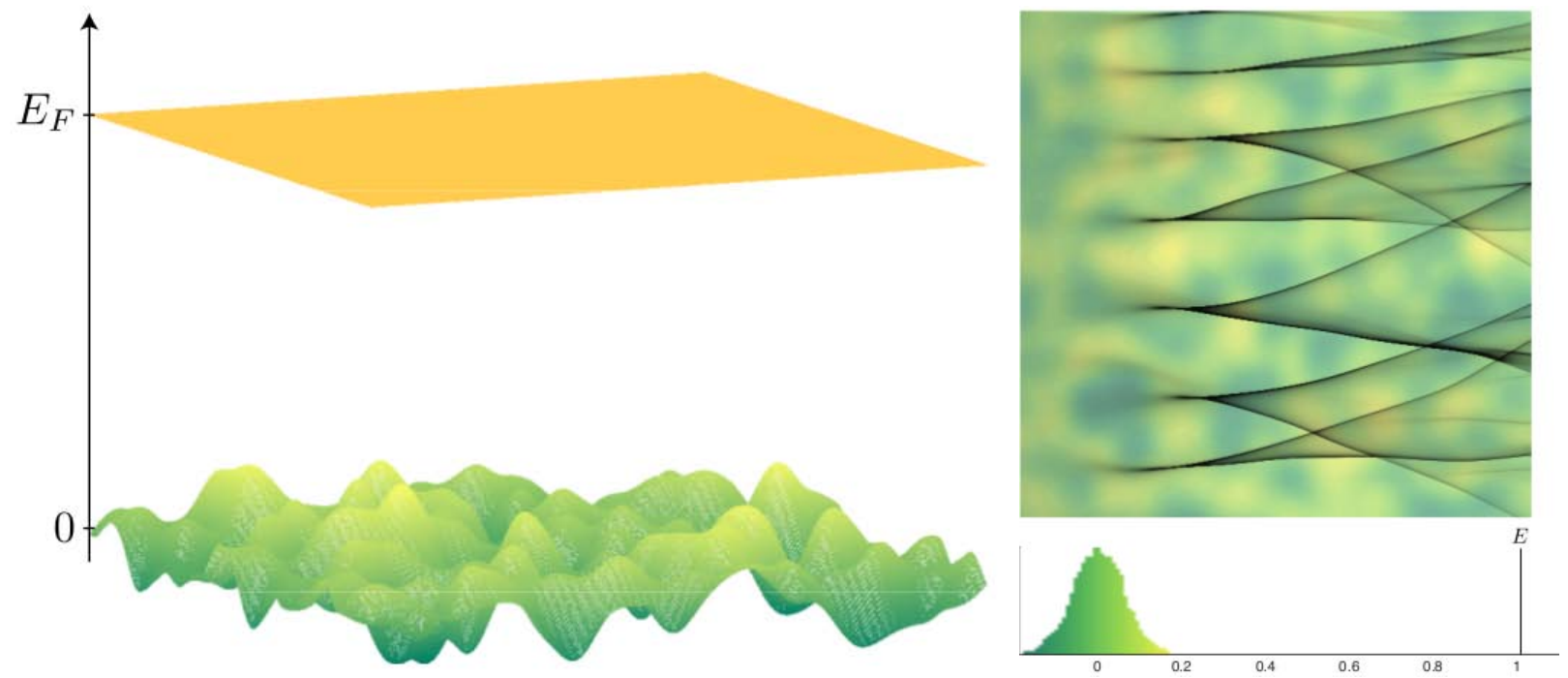

Figure 2.4: The left panel shows a random potential below the energy level of the particles, shown in orange. The right panel shows a ray simulation over a random potential, with its random caustics. Below is the distribution of the potential height compared to the energy of the particle. The potential is clearly weak compared to the energy of the particle, here normalized to one. This figure was taken from [37]

There are two main technical challenges that come with this objective. First, the curvature equation Eq.2.25 is an intricate set of nonlinear PDEs whenever the dimension of the system is larger than one. This is always the case in this thesis, as we will be interested in two dimensional systems, as tsunami waves are typically described as surface waves, see chapter 3 . Second, solving this equation for a generic random potential is generally impossible. Ideally, we would instead like to deal with an equation that is one-dimensional and can be treated stochastically, such that it would only require the knowledge of the main properties of the potential without the specifics of one realization. What we define as the quasi-2D approximation gives us exactly what we want and is a consequence of the weakness of the potential. 
The first part consists of a paraxial approximation, meaning that we consider that the rays have so much momentum in the propagation direction, compared to the weak potential, that we can neglect the small fluctuations in the propagation direction. Without the forces due to the potential, the motion of the rays in the propagation direction is simply proportional to time. For plane waves, we usually chose $x=t$ (when the velocity is $v=1$ ).

The second part concerns the potential itself. The idea is to replace it by simple white noise with a prefactor that captures the properties of the potential. The reasoning behind this is that a particle propagates so fast over the potential that it covers more than one correlation length in the propagation direction during a typical time unit. s such it essentially sees the potential as uncorrelated. Practically, we achieve this by the replacement

$$
\frac{\partial^{2}}{\partial y^{2}} V \longrightarrow \sigma_{2} \Gamma(t)
$$

where $\Gamma(t)$ is a Gaussian white noise with $\left\langle\Gamma(t) \Gamma\left(t^{\prime}\right)\right\rangle=2 \delta\left(t-t^{\prime}\right)$, which corresponds to approximating the dynamics by a diffusion process. We can understand the constant $\sigma_{2}$ in the following way: Even though the goal of this procedure is to assume a delta-correlated noise in the propagation direction $x=t$, we want to capture the properties of the random potential in the transverse direction $y$. We can achieve this by keeping the integral over the correlation functions constant, as it is related to the normalization of the potential, see 48 53 for more details. We will explain this in a simple one-dimensional picture. Let us consider a correlation function $c\left(x-x^{\prime}\right)$ that we want to approximate using white noise

$$
c\left(x-x^{\prime}\right) \longrightarrow \delta\left(x-x^{\prime}\right),
$$

but we also want to conserve the integral of the correlation function. We can achieve this by considering

$$
c\left(x-x^{\prime}\right) \longrightarrow \delta\left(x-x^{\prime}\right) \int_{-\infty}^{+\infty} \mathrm{d} x^{\prime \prime} c\left(x^{\prime \prime}\right),
$$

since this choice gives the same integral on both side of this equation. For our random potential, which is assumed to be a Gaussian random field (which allows us to take the derivatives outside of the correlation, see [54]), as discussed in appendix A, it means that the transition to white noise gives

$$
\left\langle\partial_{y y} V(x, y) \partial_{y^{\prime} y^{\prime}} V\left(x^{\prime}, y^{\prime}\right)\right\rangle=\partial_{y y} \partial_{y^{\prime} y^{\prime}} c\left(x-x^{\prime}, y-y^{\prime}\right) \longrightarrow 2 \sigma_{2}^{2} \delta\left(x-x^{\prime}\right) .
$$

This means that we find

$$
\sigma_{2}^{2}=\left.\frac{1}{2} \int_{-\infty}^{+\infty} \mathrm{d} x\left[\frac{\partial^{4}}{\partial y^{4}} c(x, y)\right]\right|_{y=0}
$$

Of course, this process does work for any derivative of the potential, as will be needed in chapters 4 and 5, as the method shown above can be applied to any randomly fluctuating term. As it will be needed later, we give here the straightforward generalization to the $n$-th derivative

$$
\sigma_{n}^{2}=\left.\frac{(-1)^{n}}{2} \int_{-\infty}^{+\infty} \mathrm{d} x\left[\frac{\partial^{2 n}}{\partial y^{2 n}} c(x, y)\right]\right|_{y=0}
$$

Using our transformed potential and studying only the transverse direction $y$, we can now rewrite the curvature equation Eq.2.25 as

$$
\frac{d}{d t} u(t)+u^{2}(t)+\sigma_{2} \Gamma(t)=0
$$


which is now an ordinary stochastic differential equation (OSDE). An OSDE with such a structure is better known as a Langevin equation. This allows us to use standard tools from stochastic calculus, including the well-known Fokker-Planck equation (F-P). This is the purpose of the next section, where these will lead us to find the mean distance that separates the first caustics from the source.

\subsection{The mean distance to the first caustics}

As earlier work [23] has shown that branch flows have a characteristic length scale that is proportional to the mean time to the first caustic, we are interested in computing the average distance that separates the source to the first caustics along a given ray or trajectory.

Mathematically, the problem is to find when the solution to Eq. 2.32 will become infinite for the first time, as a function of its initial value. This kind of problem can be handled by solving a mean first passage time problem (MFPT) with appropriate boundary conditions, which requires the introduction of an F-P equation [55]. This equation is presented in detail in appendix B and basically describes the time evolution of a probability density $P$, either backward or forward. In our case, the forward F-P equation has the form

$$
\frac{\partial}{\partial t} P(u, t)=\left[\frac{\partial}{\partial u} u^{2}+\frac{\partial^{2}}{\partial u^{2}} \sigma_{2}^{2}\right] P(u, t) .
$$

First, we have to understand what happens at the boundaries $u= \pm \infty$. The easiest way to obtain a simple understanding of these values is to first look at the curvature equation without the potential. With $\sigma_{2}=0$, Eq. 2.32 has a simple solution

$$
u(t)=\frac{1}{t+u_{0}^{-1}},
$$

such that it is straightforward to observe that with a negative $u_{0}<0, u(t)$ will reach $-\infty$ in the finite time $t=-u_{0}^{-1}$, after which it will reemerge from $+\infty$. On the other hand, a positive initial curvature $u_{0}>0$, the solution will simply flatten over time, leading to $u \rightarrow 0$. Now adding the random potential, which can diffuse the system from a positive to a negative curvature, from which it will quickly reach a caustic [49], we can better understand what are the relevant boundary conditions to solve the MFPT problem. As it is often the case with such problems, it is actually easier to solve the complementary question: What is the probability of not having a caustic until the time $t$, at which we would have $u \rightarrow-\infty$ ? In order to answer this question, we classify the boundary $u=-\infty$ as a so-called exit boundary, opposite to an entrance boundary for $u=+\infty$, according to the terminology of Feller [55, 56]. This implies the boundary conditions

$$
\begin{aligned}
& P\left(u, t \mid u_{0}\right) \rightarrow 0 \text { for } u \rightarrow-\infty \text {, } \\
& J(u, t) \rightarrow 0 \text { for } u \rightarrow+\infty,
\end{aligned}
$$

with $J(u, t)$ the probability flow as defined in appendix B, which for our F-P Eq.(2.33) is given by

$$
J(u, t)=\left[u^{2}-\frac{\partial}{\partial u} \sigma_{2}^{2}\right] P(u, t) .
$$

Now, what is the probability of no singularity appearing until the time $t$ and the process stopping there? Considering that $P\left(u, t \mid u_{0}\right)$ satisfies the above-mentionned boundary conditions Eq. 2.35, the probability would be

$$
G\left(t \mid u_{0}, t_{0}\right)=\int_{-\infty}^{+\infty} \mathrm{d} u P\left(u, t \mid u_{0}\right),
$$


and the probability of a caustic appearing at $t$ would be $1-G$. We define the probability density of this event $p_{f}$ by

$$
p_{f}\left(t \mid u_{0}\right)=-\partial_{t} \int_{-\infty}^{+\infty} \mathrm{d} u P\left(u, t \mid u_{0}\right) .
$$

This quantity satisfies the backward F-P equation (see appendix B for details)

$$
\frac{\partial}{\partial t} p_{f}=-u_{0}^{2} \frac{\partial}{\partial u_{0}} p_{f}+\sigma_{2}^{2} \frac{\partial^{2}}{\partial u_{0}^{2}} p_{f}, \lim _{t \rightarrow 0, t \rightarrow \infty} p_{f}=0 .
$$

The average time needed for $u_{0}$ to reach $u \rightarrow-\infty$ is the time to the first caustics $t_{f}$ and is given by

$$
\left\langle t_{f}\left(u_{0}\right)\right\rangle=\int_{0}^{+\infty} \mathrm{d} t t p_{f}\left(t \mid u_{0}\right) .
$$

We want to obtain a solvable equation for $\left\langle t_{f}\left(u_{0}\right)\right\rangle$ and we achieve this by multiplying Eq.2.39p by $t$ and integrating. The right hand side (r.h.s) does not contain any derivatives with respect to time and is therefore trivial. The left hand side (l.h.s), however, can be integrated immediately and yields

$$
\int_{0}^{+\infty} \mathrm{d} t t \partial_{t} p_{f}\left(t \mid u_{0}\right)=\left.t p_{f}\left(t \mid u_{0}\right)\right|_{0} ^{\infty}-\int_{0}^{+\infty} \mathrm{d} t p_{f}\left(t \mid u_{0}\right)=0-1=-1 .
$$

We can therefore write

$$
-1=-u_{0}^{2} \frac{d}{d u_{0}}\left\langle t_{f}\left(u_{0}\right)\right\rangle+\sigma_{2}^{2} \frac{d^{2}}{d u_{0}^{2}}\left\langle t_{f}\left(u_{0}\right)\right\rangle, \lim _{u_{0} \rightarrow-\infty}\left\langle t_{f}\left(u_{0}\right)\right\rangle=0, \lim _{u_{0} \rightarrow \infty}\left\langle t_{f}\left(u_{0}\right)\right\rangle=\text { finite . }
$$

This is a straightforward second order ODE that can be integrated as follows. We first define new variables $x:=u_{0}$ and $y=\frac{d}{d u_{0}}\left\langle t_{f}\left(u_{0}\right)\right\rangle$. With these new variables, we have the first order ODE

$$
\sigma_{2}^{2} y^{\prime}=x^{2} y-1
$$

which can be solved easily by the method of the variation of the constant, yielding

$$
y=C e^{x^{3} / \sigma_{2}^{2}}-\frac{e^{x^{3} / 3 \sigma_{2}^{2}}}{\sigma_{2}^{2}} \int_{x}^{\infty} e^{-z^{3} / 3 \sigma_{2}^{2}} \mathrm{~d} z .
$$

We set $C=0$ to satisfy the boundary conditions and we can integrate once more to obtain the solution for the time to the first caustics (once we have transformed back to our previous variables)

$$
\left\langle t_{f}\left(u_{0}\right)\right\rangle=\frac{1}{\sigma_{2}^{2}} \int_{-\infty}^{u_{0}} e^{w^{3} / 3 \sigma_{2}^{2}} \int_{w}^{+\infty} e^{-z^{3} / 3 \sigma_{2}^{2}} \mathrm{~d} z \mathrm{~d} w .
$$

These integrals can be calculated numerically depending on the needed initial condition, typically 0 or $\infty$, respectively for plane waves and point sources. It is given by 49,57 as

$$
\left\langle t_{f}(0)\right\rangle=3.31 \sigma_{2}^{-2 / 3} \text { and }\left\langle t_{f}(\infty)\right\rangle=4.97 \sigma_{2}^{-2 / 3} .
$$

As the velocity was chosen to be $v=1$, this time to the first caustic also corresponds to the distance to the first caustics.

This analytical result was confirmed numerically in fully two-dimensional simulations in [37. 


\subsection{Conclusion}

In this chapter, we presented the basics of the theory of caustics and branched flow by studying the emergence of multivalued solutions to the Hamilton-Jacobi equation, leading to a divergence of the curvature of the action. We also showed that the curvature is closely related to the flow density, which classically diverges at the focus points. The study of the curvature equation in the so-called quasi-2D approximation allowed us to derive an analytical prediction for the distance to the first caustics in the case of weak random focusing. We explained why this quantity is crucial in the study of branched flow, as it corresponds to the location where the intensities are the strongest and it is the characteristic length-scale of this phenomenon, with which many important quantities scale.

With the help of these methods, we will now study the emergence of branched flows in tsunami propagation, as can be anticipated from Fig 1.1 . Before we can apply all these theoretical tools, we will first need to lay down the basics about tsunami modeling, in order to find a good common framework, namely ray equations. This is the purpose of the next chapter. 


\section{Chapter 3}

\section{Modeling tsunami waves}

In the previous chapters, we presented the phenomenology and theory of branched flow and explained why we believe it to be relevant in tsunami studies, through the simulation produced by the NOAA in 2011 (see Fig 1.1). In order to obtain a theoretical understanding of how random focusing affects tsunami propagation, we need a mathematical description of tsunami waves and, in order to study the appearance of caustics, the associated rays. This is the purpose of this chapter.

\subsection{Phenomenology}

Tsunami waves are gigantic water waves induced by very powerful geological disasters like earthquakes or landslides 58. Even though there are records of small tsunami events happening in the Mediterrean sea, most tsunamis of interest occur in the ocean. Their wavelength is considered to range from a few tens to a few hundreds of kilometers [58], with a period of several tens of minutes, and the amplitude is typically of the order of 1 meter [58] far from the shore, before the run-up occurs (see section 3.3). As those waves are relatively long compared to the average depth of the ocean, which is around 4 kilometers, the shallow water approximation applies.

We note here that due to the fact that they propagate on a spinning Earth, tsunami waves are subject to the Coriolis force. Also, the curvature of the Earth has to be considered for long range propagation. However, we will neglect these two effects throughout this work, as is done in many tsunami studies. Indeed, the Coriolis force is very weak, even at the latitudes were it is the strongest. An estimate of its importance is given in [59], where they evaluate the error made to $10^{-4}$. As for the curvature, neglecting it leads to an error that increases with the propagation distance. An estimate can be made considering that the usual flat metric should be replaced by a spherical metric 59]

$$
\mathrm{d} x^{2}+\mathrm{d} y^{2} \longrightarrow r \mathrm{~d} \theta^{2}+r \cos ^{2}(\theta) \mathrm{d} \phi^{2},
$$

meaning that, when considering propagation over a couple of thousands of kilometers, the errors made on distances is only of a few percent 59 .

\subsection{The shallow water equations}

The shallow water approximation, also know as the long wave approximation, is based on the idea that the typical horizontal scale of the wave $\lambda$ (the wavelength) is much larger than the typical vertical scale of the system $H_{0}$ (the average depth of the fluid). This condition is largely met when dealing with tsunami waves, as we typically have $\lambda / H_{0} \approx 100 \mathrm{~km} / 4 \mathrm{~km}=25 \gg 1$. It can be shown that when the ratio $\lambda / H_{0} \gg 1$, the hydrostatic equilibrium approximation applies in the vertical direction $z$ and we have 60,61 


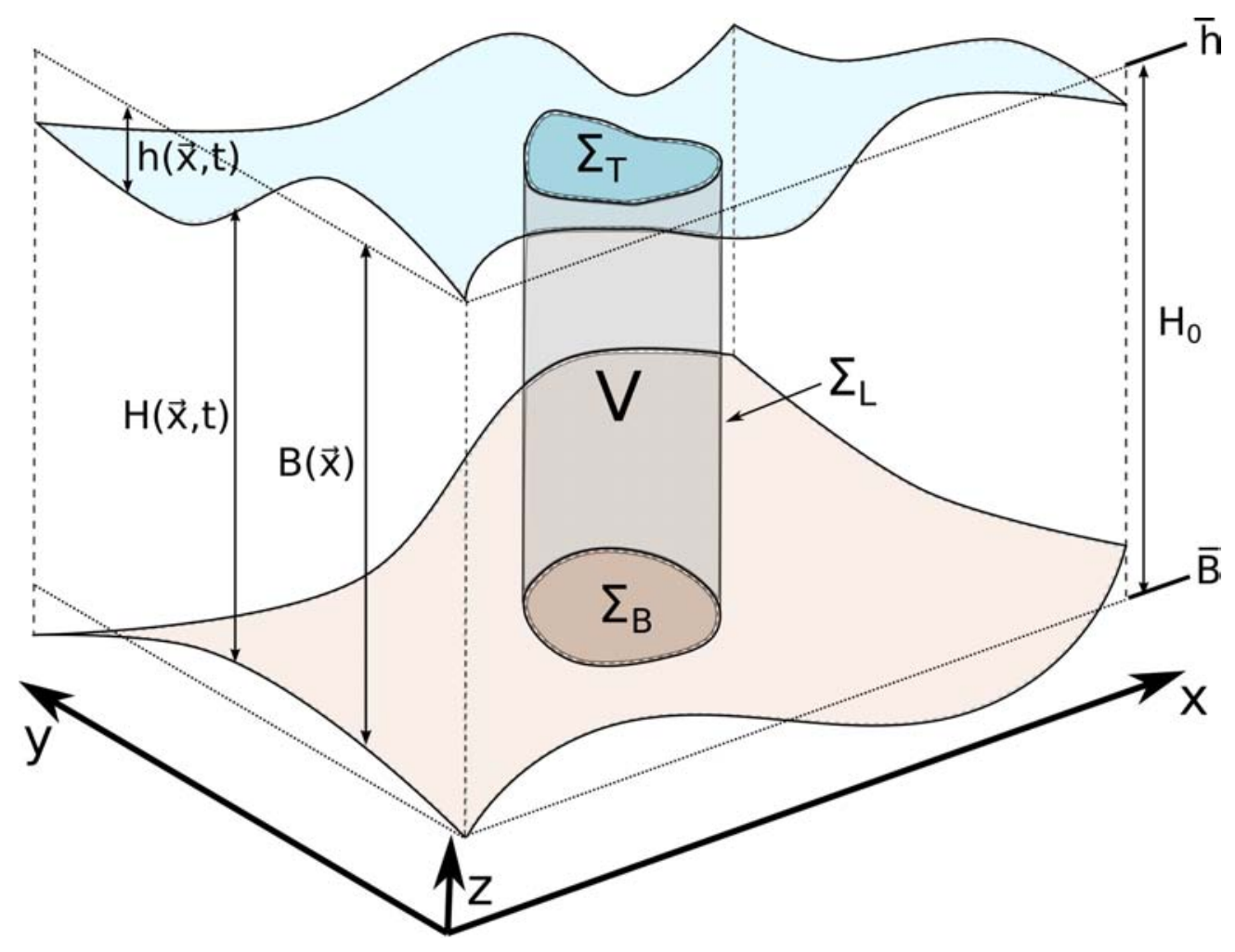

Figure 3.1: This is a scheme of the fluid under consideration. The bottom sheet (brown) represents the floor (bathymetry) and the top sheet (blue) represents the surface of the fluid. The volume $\mathrm{V}$ is framed by the bottom surface $\Sigma_{B}$, the top surface $\Sigma_{T}$ and the lateral surface $\Sigma_{L}$. The values $\bar{h}$ and $\bar{B}$ are respectively the average level of the surface of the fluid (its position at rest) and the average elevation of the floor. The difference between these two values therefore defines the average depth $H_{0}$. The function $h(x, y, t)$ describes the surface elevation. $B(x, y)$ is the bathymetry, measured from $\bar{h} . H(x, y, t)=h(x, y, t)-B(x, y)$ is the actual height of the fluid column. Here $\vec{x}$ stands for the horizontal space $(x, y)$.

$$
p(x, y, z, t)=\rho g[h(x, y, t)-z],
$$

where $p(x, y, z, t)$ is the pressure, $\rho$ is the density of the fluid (assumed to be constant), $g$ is the gravitational acceleration and $h(x, y, t)$ is the function describing the surface of the fluid.

Another consequence of the hydrostatic equilibrium approximation, along with the incompressibility of the fluid, is that the fluid velocity $\vec{u}=(u, v, w)$ is independent of $z$ and its vertical component $w=0$, see 61] for details.

Even though the shallow water equations are well-known and have been studied in many papers (not necessarily related to tsunami waves), we will present here their derivation, based on conservation laws, following [60], because we will have to study variations of it later in this thesis, when dealing with the prospect of the experiment (see chapter 6).

We start by considering a single layer of incompressible fluid that is bounded by the ocean floor or bathymetry $B(x, y)$ from the bottom and by the ocean surface $h(x, y)$ from the top. We consider a column of fluid of volume $V$, that is bounded by a surface $\Sigma=\Sigma_{B} \cup \Sigma_{T} \cup \Sigma_{L}$, as shown in Fig. 3.1. This volume is subject to conservation of mass

$$
\partial_{t} \int_{V} \rho \mathrm{d} V=\int_{\Sigma} \rho \vec{u} \cdot \vec{n} \mathrm{~d} S,
$$

with $\vec{n}$ the unit outward normal vector to $\Sigma$. We use the symbolic differentials $\mathrm{d} V$ for volume integration, $\mathrm{d} S$ for surface integration and finally $\mathrm{d} \gamma$ for curvilinear integration. As the velocity 
has to be tangential to the floor and the surface of the fluid by definition, the surface integrals for $\Sigma_{T}$ and $\Sigma_{B}$ will vanish. After getting rid of the constant density $\rho$, we can integrate over $z$ to obtain

$$
\partial_{t} \int_{D} H(x, y, t) \mathrm{d} S=\oint_{\partial D} H(x, y, t) \vec{u}_{\perp} \cdot \vec{n}_{\perp} \mathrm{d} \gamma,
$$

where $D$ is the projection of $\Sigma_{B}$ (and $\Sigma_{T}$ by extension) on the $x y$-plane, $\partial D$ is the boundary of the domain $D, \vec{u}_{\perp}$ is the horizontal velocity, $\vec{n}_{\perp}$ is the horizontal unit vector in the outward direction of $\partial D$ and $H(x, y, t)=h(x, y, t)-B(x, y)$ is the height of the water column, as can be seen in Fig 3.1 .

This volume is also subject to conservation of momentum, which reads

$$
\partial_{t} \int_{V} \rho \vec{u}_{\perp} \mathrm{d} V=-\int_{\Sigma_{L}}\left(\rho \vec{u}_{\perp}\right) \vec{u} \cdot \vec{n} \mathrm{~d} S-\int_{\Sigma_{L}} p(x, y, z, t) \vec{n}_{\perp} \mathrm{d} S-\int_{\Sigma_{B}} p(x, y, z, t) \vec{n}_{\perp} \mathrm{d} S,
$$

where the constant pressure term from the top surface was set to zero, since we define the pressure to be zero at this level. We note that the pressure won't be constant and can't be simply set to zero at the surface when dealing with surface tension, see chapter 6 . The vertical forces are neglected, as they are assumed to balance due to the hydrostatic equilibrium hypothesis [61]. We can now use the expression for the pressure defined in Eq. (3.2) and integrate over $z$ to obtain

$$
\partial_{t} \int_{D} \rho H \vec{u}_{\perp} \mathrm{d} S=-\oint_{\partial D}\left(\rho H \vec{u}_{\perp}\right) \vec{u}_{\perp} \cdot \vec{n}_{\perp} \mathrm{d} \gamma-\oint_{\partial D} \frac{1}{2} \rho g H^{2} \vec{n}_{\perp} \mathrm{d} \gamma-\int_{D} \rho g H \nabla_{\perp} B \mathrm{~d} S,
$$

with $\nabla_{\perp}:=\left(\partial_{x}, \partial_{y}\right)^{T}$. In the last integral, the gradient of $B$ comes from the reparametrization that occurs when while changing the integral domain from $\Sigma_{B}$ to $D, H$ comes from the fact that $H=h-z$ on $\Sigma_{B}$. We dropped the dependencies on the variables in order to keep it concise. We can once again divide out the constant density $\rho$.

Using Stoke's theorem in Eq.(3.4) and Eq.(3.6) and going from integral to differential form, we obtain the well-known shallow water equations

$$
\begin{aligned}
\partial_{t} H+\partial_{x}(H u)+\partial_{y}(H v) & =0, \\
\partial_{t}(H u)+\partial_{x}\left(H u^{2}+\frac{1}{2} g H^{2}\right)+\partial_{y}(H u v) & =-g H \partial_{x} B \\
\partial_{t}(H v)+\partial_{x}(H u v)+\partial_{y}\left(H v^{2}+\frac{1}{2} g H^{2}\right) & =-g H \partial_{y} B .
\end{aligned}
$$

\subsection{Dimensionless form and linearization}

Now that we have the shallow water equations Eqs.(3.7), we want to write them with dimensionless quantities, as it will render them less unwieldy and will simplify the comparison between the different terms. We achieve this by canceling out one of the main parameters of the system, namely the average ocean depth $H_{0}$. We therefore define the fractional surface elevation

$$
\eta(x, y, t)=H_{0}^{-1} h(x, y, t),
$$

and the reduced bathymetry $\beta$, which is the centered, normalized version of $B$ (which, we remind the reader, is measured from the sea level and is therefore negative in the ocean)

$$
\beta(x, y)=H_{0}^{-1}\left[B(x, y)+H_{0}\right]
$$


as can be seen in Fig 3.2 . We note that $\eta$ is a very small quantity, as the typical wave height $h$ is of the order of one meter and the depth of the ocean, $H_{0}$, is typically of the order of few kilometers, meaning that $\eta \sim 10^{-4}$. Also, we will restrict ourselves to the cases where $\beta<1$, i.e. there are no islands, as we want to study the linear regime far from the coasts. With those new

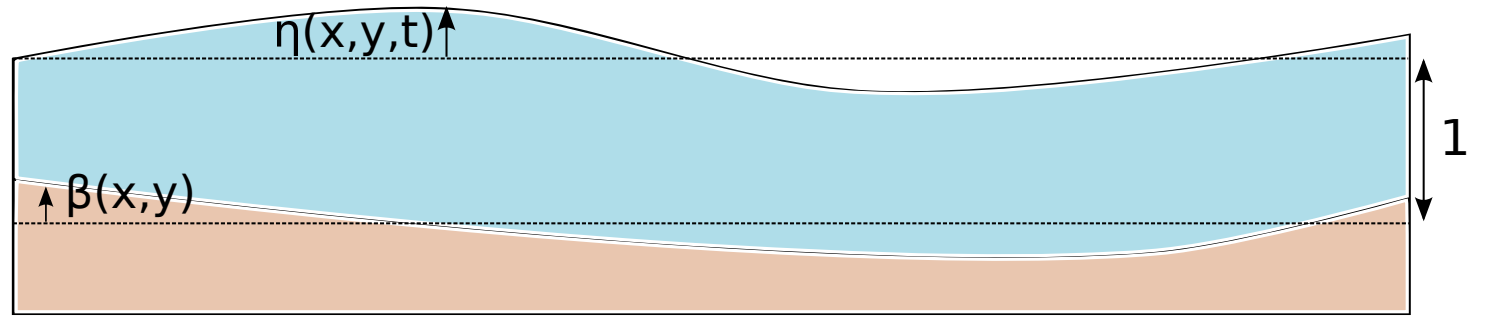

Figure 3.2: This scheme defines the new dimensionless variables. The average ocean depth is now 1 and $\beta$ is positive when above the average depth.

variables, the total height of the water column $H(x, y, t)$ is given by

$$
H(x, y, t)=H_{0}(1+\eta(x, y, t)-\beta(x, y)) .
$$

Of course, as the wavelength $\lambda$ is much larger than the ocean depth $H_{0}$, the spatial derivatives of $\eta$ will also be very small, as will be its time derivative. Looking at Eq. (3.7) with this new set of variables, we have:

$$
H_{0} \partial_{t} \eta=H_{0}(1+\eta-\beta) \nabla \cdot \vec{u}+H_{0} \vec{u} \cdot \nabla(\eta-\beta),
$$

where we define $\vec{u}=(u, v)^{T}$, the two-dimensional velocity, and we recall that we are now working in two spatial dimensions. We can divide by $H_{0}$ and neglect the nonlinear terms that contain a factor of $\eta$ or its derivatives to obtain

$$
\partial_{t} \eta=(1-\beta) \nabla \cdot \vec{u}-\vec{u} \cdot \nabla \beta .
$$

In order to get an estimate of the strength of the terms in $\vec{u}$, let's consider $\beta=0$, i.e. a flat ocean floor,

$$
\partial_{t} \eta=\nabla \cdot \vec{u}
$$

We see that the velocity terms are on the same order as the wave height terms, we therefore assume that they are small as well. With this, we can linearize Eqs.3.7) to obtain

$$
\begin{aligned}
\partial_{t} \eta & =-\nabla \cdot[(1-\beta) \vec{u}] \\
\partial_{t} \vec{u} & =-c_{0}^{2} \nabla \eta,
\end{aligned}
$$

where we introduced the celerity of the linear waves $c_{0}=\sqrt{g H_{0}}$, which is typically of the order of $200 \mathrm{~m} / \mathrm{s}$ in a $4 \mathrm{~km}$ deep ocean. Those equations can of course be combined to form a single wave equation

$$
\partial_{t}^{2} \eta=c_{0}^{2} \nabla \cdot[(1-\beta) \nabla \eta] .
$$

As we will see in the next section, it is common to work in the geometrical optics limit, which is an approximation in which we assume that the medium term (here, the reduced bathymetry $\beta$ ) is slowly varying in space compared to the wave. This can be justified by the fact that the scale at which the variations in the bathymetry produce wave focusing will be larger than the wavelength $\lambda$, as a first approximation. We compared the intensity of waves propagating over bathymetries that have a typical length scale which is either shorter or longer than the wavelength and we see much higher wave intensities when the bathymetry varies on a scale larger than the wavelength, see Fig 3.4. Formally, we consider $\lambda \ll l_{c}$, where $l_{c}$ is the correlation length of the bathymetry, i.e. 
its typical horizontal length scale. As we will also be considering weakly scattering ocean floors, $\beta \ll 1$, the spatial derivatives of the bathymetry will thus be very small. Under these considerations, Eq. (3.15) becomes

$$
\partial_{t}^{2} \eta=c_{0}^{2}[1-\beta] \Delta \eta
$$

We can now see an obvious similarity with optics, where the term $C(\vec{x})=c_{0} \sqrt{(1-\beta(\vec{x}))}$ can be seen as a space-dependent celerity where $n(\vec{x})=(1-\beta(\vec{x}))^{-1 / 2}$ would act as a refractive index. This wave equation structure leads to the so-called run-up, that is illustrated in Fig 3.3. This effect explains how tsunamis change from meter-high waves in the middle of the ocean to 10-meter-high (or even higher) waves on the coast. As this mechanism amplifies the size of the wave, it is still very important to known how high the waves are going to be when this amplification starts. It is of course also very relevant to know where the highest waves are to be expected, because, once amplified, these are the waves that are going to be the most destructive. It is one of the questions we will answer in this work.

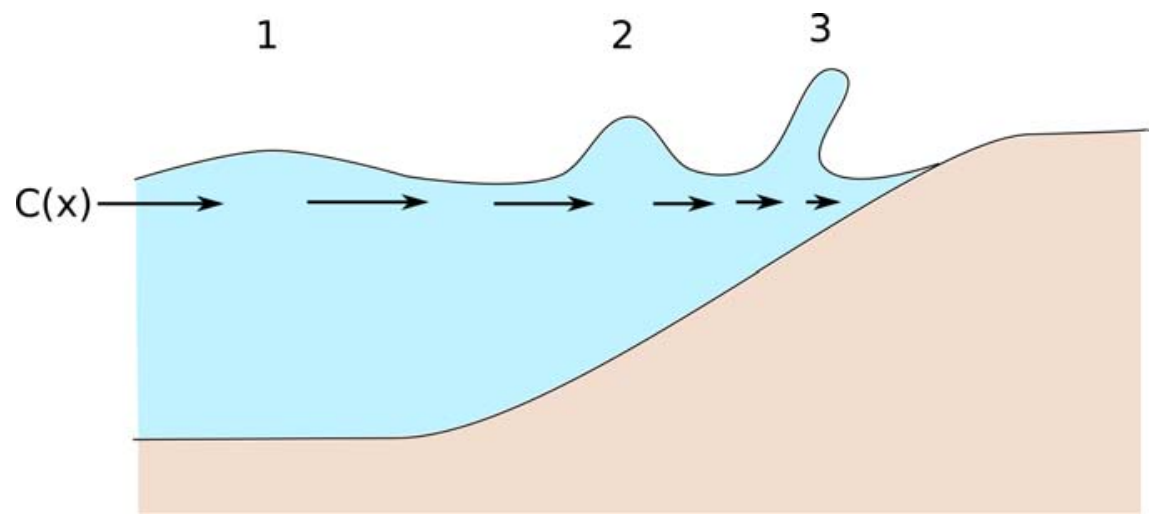

Figure 3.3: The run-up - As a tsunami wave is approaching the shore (1), the bathymetry becomes higher and higher, therefore making the effective celerity $C(\vec{x})$ smaller and smaller. The evolution of $C(\vec{x})$ is represented in the length of the arrows. The fact that the back part of the wave "catches up" with the front part of the wave results in the compression of the wave (2). Eventually, the wave will become high enough and the fluid shallow enough for the nonlinearity to become important, resulting in the well-known breaking of the wave(3).

Furthermore, large underwater structures, such as volcanoes and ridges, will act as lenses, scattering and eventually focusing the waves, as was studied in [6]. Our goal in this thesis is to study the effect that a random field of weak scatterers in the bathymetry would have on the intensity of the tsunami waves, as random focusing in weakly scattering, correlated media is known to lead to branched flows. In order to investigate it, we need to understand the emergence of caustics in the wave flow. As explained in chapter 2, this problem is well-defined in the associated ray picture. In the next section, we shall, therefore, derive the ray equations associated with Eq. 3.15).

\subsection{Shallow water rays}

When waves propagate through a medium which varies on several length scales, there will be different types of scattering, depending on the relative size of the wave and the scatterer. Typically, when a wave encounters a scatterer the size of which is smaller than the wavelength, the wave will be diffused and the scatterer will become the source of a new wave. Considering a random field of such scatterers would lead to a random superposition of waves, therefore leading to a Rayleigh distributed wave intensity, as predicted by Longuet-Higgins' theory [62]. On the other hand, when the size of the scatterer is larger than the wave length, we are in the geometrical optics limit and we all know by experience that waves will tend to be bent and focused. 

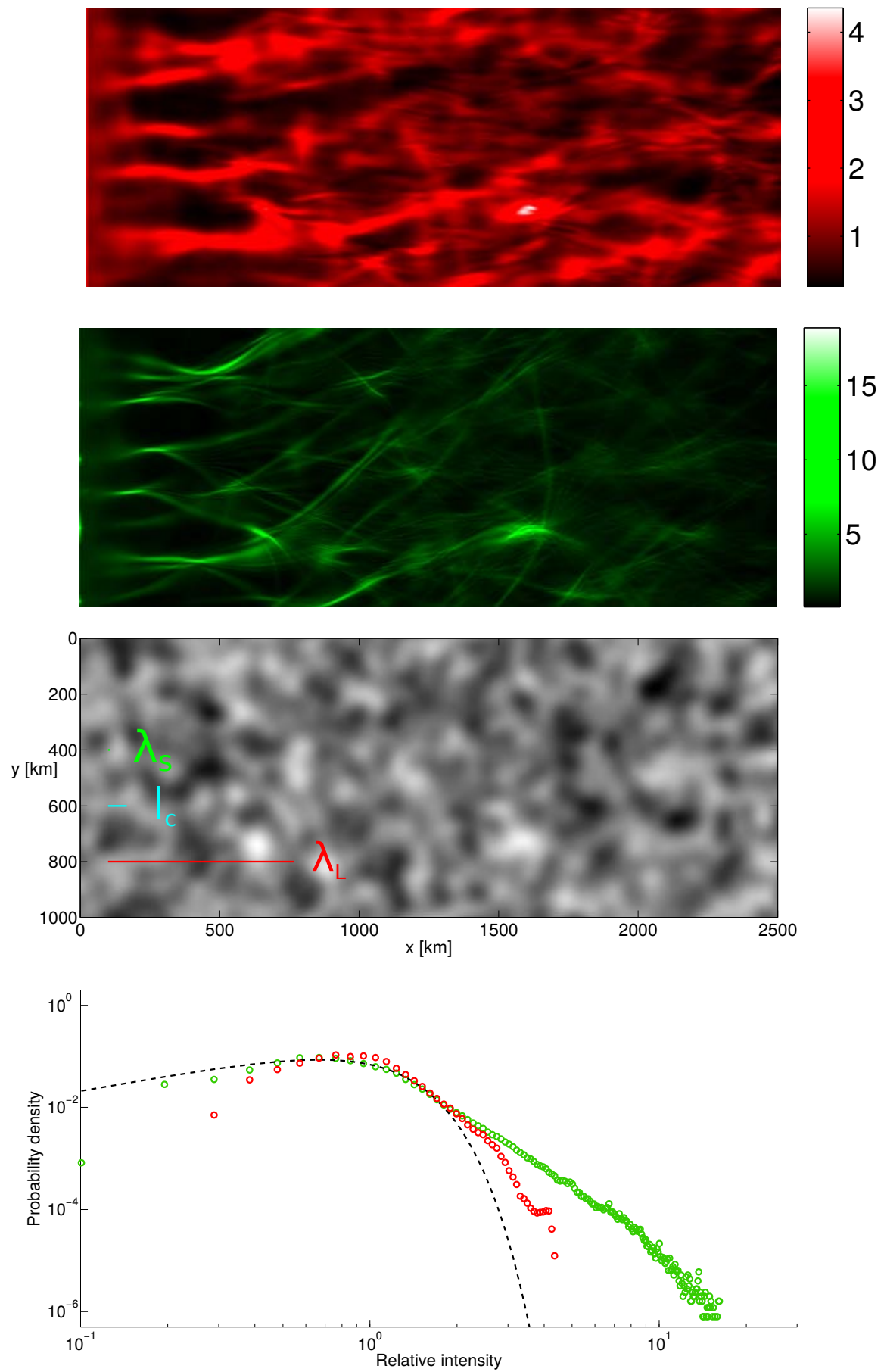

Figure 3.4: On the second bottom panel, we can see a random bathymetry (gray colormap), on which we evolved two plane waves with different wave lengths, the intensities of which can be seen on the two top panels. On this bathymetry, the correlation length $l_{c}$ (cyan), the short wave length $\lambda_{s}=l_{c} / 10$ (green) and the long wave length $\lambda_{L}=10 l_{c}$ (red) are drawn. The top panel shows the normalized integrated intensities for the experiment with a wave length $\lambda_{L} \gg l_{c}$. We see that the patterns are very blurry and the highest intensities are of the order of 4 times the average, as can be seen in the color bar. The second top panel shows the same experiment, but this time with a short wavelength $\lambda_{s} \ll l_{c}$. In this case, the branched flow patterns appear very clearly and we observe intensities as high as 18 times the average! The bottom shows the distribution of intensities for both experiments, the green dots corresponding to the $\lambda_{s}$ case and the red dots corresponding to the $\lambda_{L}$ case. The black dashed curve represents the Rayleigh distribution predicted by the Longuet-Higgins model, as a reference. We can clearly see that the tail is much heavier for the short wavelength case. 
As the ocean floor is known to have multiple length scales [63], both effects will be present. Nevertheless, as the diffused waves will contribute Rayleigh noise to the system (as a first approximation), we will only consider the fluctuations of the ocean floor that are on scales larger than the wavelength, because they are the ones responsible for the focusing of the wave and therefore the appearance of the biggest intensities, see Fig 3.4 .

Starting from our wave equations in the geometrical optics limit (Eq.(3.16)), we will derive ray equations by looking at vectors orthogonal to the wave front, following [59]. The wave equation can be written as

$$
\left(\partial_{t}^{2}-c_{0}^{2}(1-\beta(\vec{x})) \Delta\right) \eta(\vec{x}, t)=0 .
$$

Because the space-dependent celerity $C(\vec{x})=c_{0} \sqrt{1-\beta(\vec{x})}$ is time-independent, the solution is a linear combination of waves with specific frequencies and amplitudes, which can be formulated in the following way 59

$$
\eta(\vec{x}, t)=A(\vec{x}) \cos (L(\vec{x})-\omega t),
$$

where $\omega$ is a frequency and $A$ and $L$ are functions of space. The function $\Phi(\vec{x}, t)=L(\vec{x})-\omega t$ defines the phase of the wave, and locations in space with equal phases will represent a wave front. We want to identify a specific wave front, so let us choose the one at $\Phi=0$, such that

$$
L(\vec{x})=\omega t .
$$

Since we want to find the vectors orthogonal to the wave front, we will need the same wave front at a short time $\delta t$ later, as sketched in Fig 3.5 . We pick $\vec{x}_{r}(t)$ a point of this wavefront at time $t$

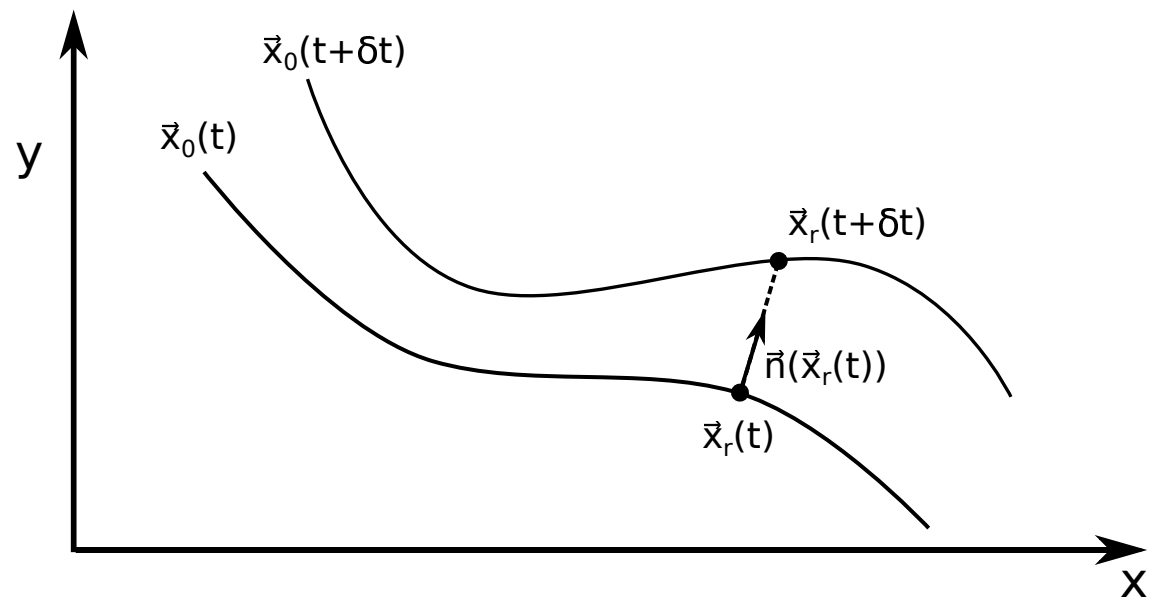

Figure 3.5: We can here see the sets of points of phase zero at time $t, \vec{x}_{0}(t)=\left\{\vec{x} \in \mathbb{R}^{2} \mid \Phi(\vec{x}, t)=0\right\}$, and its updated version at time $t+\delta t$. We choose a point $\vec{x}_{r}(t) \in \vec{x}_{0}(t)$ and then define $\vec{x}_{r}(t+\delta t)$ as the point at which the straight line defined by the normal $\vec{n}\left(\vec{x}_{r}\right)$ intersects $\vec{x}_{0}(t+\delta t)$.

$$
L\left(\vec{x}_{r}(t)\right)=\omega t
$$

so the normal $\vec{n}\left(\vec{x}_{r}(t)\right)$ to the wave front at this point is given by

$$
\vec{n}\left(\vec{x}_{r}(t)\right)=\left.\nabla L\right|_{\vec{x}=\vec{x}_{r}(t)} .
$$

As shown in Fig 3.5 , we now consider the point $\vec{x}_{r}(t+\delta t)$ that is at the intersection of $\vec{x}_{0}(t+\delta t)$ and the line defined by $\vec{n}$. Then we can write

$$
\omega(t+\delta t)=L\left(\vec{x}_{r}(t+\delta t)\right)=L\left(\vec{x}_{r}(t)+\delta l \hat{n}\left(\vec{x}_{r}(t)\right)\right)=L\left(\vec{x}_{r}(t)\right)+\delta l \hat{n}\left(\vec{x}_{r}(t)\right) \cdot \vec{n}\left(\vec{x}_{r}(t)\right)
$$


where $\delta l$ is the infinitesimal displacement made and $\hat{n}\left(\vec{x}_{r}(t)\right)$ is the unit vector in the direction of $\vec{n}\left(\vec{x}_{r}(t)\right)$. With this, we can define the ray celerity $C_{r}$, which is given by

$$
C_{r}\left(\vec{x}_{r}(t)\right):=\frac{\delta l}{\delta t}=\frac{\omega}{\left|\vec{n}\left(\vec{x}_{r}(t)\right)\right|},
$$

thus giving

$$
\frac{d}{d t} \vec{x}_{r}=C_{r}\left(\vec{x}_{r}(t)\right) \hat{n}\left(\vec{x}_{r}(t)\right) .
$$

The knowledge of $\Phi$ is the only thing needed to compute $\vec{n}\left(\vec{x}_{r}(t)\right)$. One can understand Eq.3.24 as describing a ray traveling at the speed $C_{r}\left(\vec{x}_{r}(t)\right)$.

Now, we can insert our ansatz Eq. (3.18) in Eq. (3.17) in order to find the function $L$, which is the only thing needed to find $\Phi$. As discussed earlier, the amplitude $A$ of the wave is very small, as are its derivatives. We will therefore neglect those terms. This yields

$$
|\nabla L|^{2}=\frac{\omega^{2}}{C^{2}}
$$

where we recall that $C(\vec{x})=c_{0} \sqrt{1-\beta(\vec{x})}$. This shows that

$$
C_{r}\left(\vec{x}_{r}\right)=C\left(\vec{x}_{r}\right),
$$

using Eq. 3.23). Of course, with a given floor $\beta$, we could try to solve Eq.(3.25), but this is often not possible. Instead, we can obtain ray equations by using Eq.(3.25) in Eq.(3.24) to write

$$
\frac{\omega}{C^{2}} \frac{d}{d t} \vec{x}_{r}=\nabla L\left(\vec{x}_{r}\right),
$$

since

$$
\hat{n}\left(\vec{x}_{r}(t)\right)=\left.\left(\frac{\nabla L}{|\nabla L|}\right)\right|_{\vec{x}=\vec{x}_{r}(t)} \text { and }|\nabla L|=\frac{\omega}{C} .
$$

Eq. 3.27 can be differentiated with respect to time

$$
\frac{d}{d t} \frac{\omega}{C^{2}} \frac{d}{d t} \vec{x}_{r}=\frac{d}{d t} \nabla L\left(\vec{x}_{r}\right) .
$$

In order to find an expression for the r.h.s. that does not need to be solved for $L$, we can differentiate Eq. 3.25 to find

$$
2 \nabla L \cdot\left(\frac{d}{d t} \nabla L\right)=\frac{d}{d t}|\nabla L|^{2}=\omega^{2} \frac{d}{d t}\left[C^{-2}\right]=-\frac{2 \omega^{2}}{C^{2}}\left(\frac{d}{d t} \vec{x}_{r}\right) \cdot(\nabla \ln C) .
$$

Together with Eq.(3.27), it yields

$$
\frac{d}{d t} \nabla L=-\omega \nabla \ln C
$$

With this, we can write

$$
\frac{d}{d t} C^{-2} \frac{d}{d t} \vec{x}_{r}=-\nabla \ln C,
$$

which can be rewritten as a set of first order ordinary differential equations, where, from now on, we will use $\vec{x}$ instead of $\vec{x}_{r}$ for simplicity,

$$
\begin{aligned}
\frac{d}{d t} \vec{x} & =C^{2} \vec{q}, \\
\frac{d}{d t} \vec{q} & =-\nabla \ln C .
\end{aligned}
$$


Finally, using our definition of $C=c_{0} \sqrt{1-\beta}$, we find

$$
\begin{aligned}
\frac{d}{d t} \vec{x} & =c_{0}^{2}(1-\beta(\vec{x})) \vec{q}, \\
\frac{d}{d t} \vec{q} & =\frac{\nabla \beta(\vec{x})}{2(1-\beta(\vec{x}))} .
\end{aligned}
$$

We note here that the variable $\vec{q}$, which acts as a momentum in the ray equations, has the unit of a slowness $[q]=s m^{-1}$. In order to fix this unnatural choice, we define the momentum $\vec{p}=c_{0}^{2} \vec{q}$, which yields

$$
\begin{aligned}
\frac{d}{d t} \vec{x} & =(1-\beta(\vec{x})) \vec{p}, \\
\frac{d}{d t} \vec{p} & =\frac{c_{0}^{2} \nabla \beta(\vec{x})}{2(1-\beta(\vec{x}))} .
\end{aligned}
$$

\subsection{Conclusion}

In this chapter, we derived the linearized shallow water equations in order to model tsunami propagation, using a few approximations. This gave us a fairly simple wave equation in terms of our dimensionless variables. As one would do in optics, we studied the evolution of vectors perpendicular to the wavefront in order to derive ray equations, which are the basis of our analysis of the appearance of caustics. With these equations, we can investigate the emergence of branched flow in this system, the length scale on which this phenomenon takes place and its impact on tsunami forecasts. It is the topic of our next chapter. 



\section{Chapter 4}

\section{Branching of tsunami waves}

Now that we have introduced an appropriate model to describe tsunami waves in deep ocean conditions and recapitulated the theory of random caustics and branched flow, we can finally enter the heart of this thesis: How are tsunami waves affected by the phenomenon of branched flow?

We first show that small bathymetry fluctuations indeed lead to focusing of the tsunami wave in a branched structure. We will then develop the theory of random caustics for tsunami rays and calculate the typical length scale at which the first caustics appear. We will show that strong height fluctuations of tsunami waves indeed appear on this length scale and that the random focusing of tsunami waves is in fact caused by the phenomenon of branching described in chapter 2 . We conclude by questioning our current capacity to forecast tsunami disasters: by studying the impact of uncertainties in bathymetry data on the predictability of branching patterns, we show that the current knowledge of the ocean floor topography is not accurate enough for reliable tsunami forecasts.

\subsection{Branching on a real floor}

In chapter 1 , we showed the results of a numerical reconstruction of the tsunami that occurred in March 2011 in the Pacific ocean (see Fig 1.1). We pointed out that the tsunami intensities exhibited strong fluctuations and a filamentation of the flow and discussed the possibility that this phenomenon could be related to the emergence of random caustics leading to the branching of the flow, described in more details in chapter 2. As we know from the previous chapter that shallow water waves, which is the model used to describe tsunamis, are scattered by underwater structures, we could conclude that the filamentation of the flow that is observed in Fig 1.1 is due to the fluctuations in the bathymetry. However, as this simulation covers a vast region of the Pacific ocean which is filled with bathymetry structure of all sizes, ranging from flat valleys to sharp islands, it is difficult to know what type of scattering process is responsible for the variations in the flow intensity. The origins of this filamentation are probably various and it is therefore difficult to impute the observed branch-like patterns to actual branched flow, i.e. to weak and correlated random focusing.

In order to show whether or not shallow water waves do exhibit actual branched flow and therefore are strongly focused by weak fluctuations in the bathymetry, we wanted to simulate a tsunami event in a region of the ocean where there would be no doubt that, if there is a filamentation of the flow, it is due to random focusing by small structures. We were therefore looking for a region that is both relevant for tsunami propagation (typically, in the Indian or Pacific ocean) and weakly scattering (i.e. has no large vertical structure like islands), as we want to see if the patterns of intensity fluctuations can be imputed to weak random focusing. We decided to use the GEBCO database [64], which contains the known topography of the Earth, both for the surface (continental) and the ocean floor (bathymetry), and is publicly available. We found a region in the Indian ocean with a standard deviation in the elevation, i.e. the typical 
vertical size of the underwater structures, of only $\sim 7 \%$. Its location is shown in Fig 4.1 . When we look at this region in a three-dimensional picture, as in Fig 4.2 , we see that only a few hills reach heights bigger than $15 \%$ of the ocean depth. The largest structure is a well within $-60 \%$. We had to plot this region with a powerful magnification of the vertical direction, because plotting it up-to-scale would produce a depth-profile that would seem totally flat, as the horizontal scales at which the structure varies are much larger than the vertical scales.

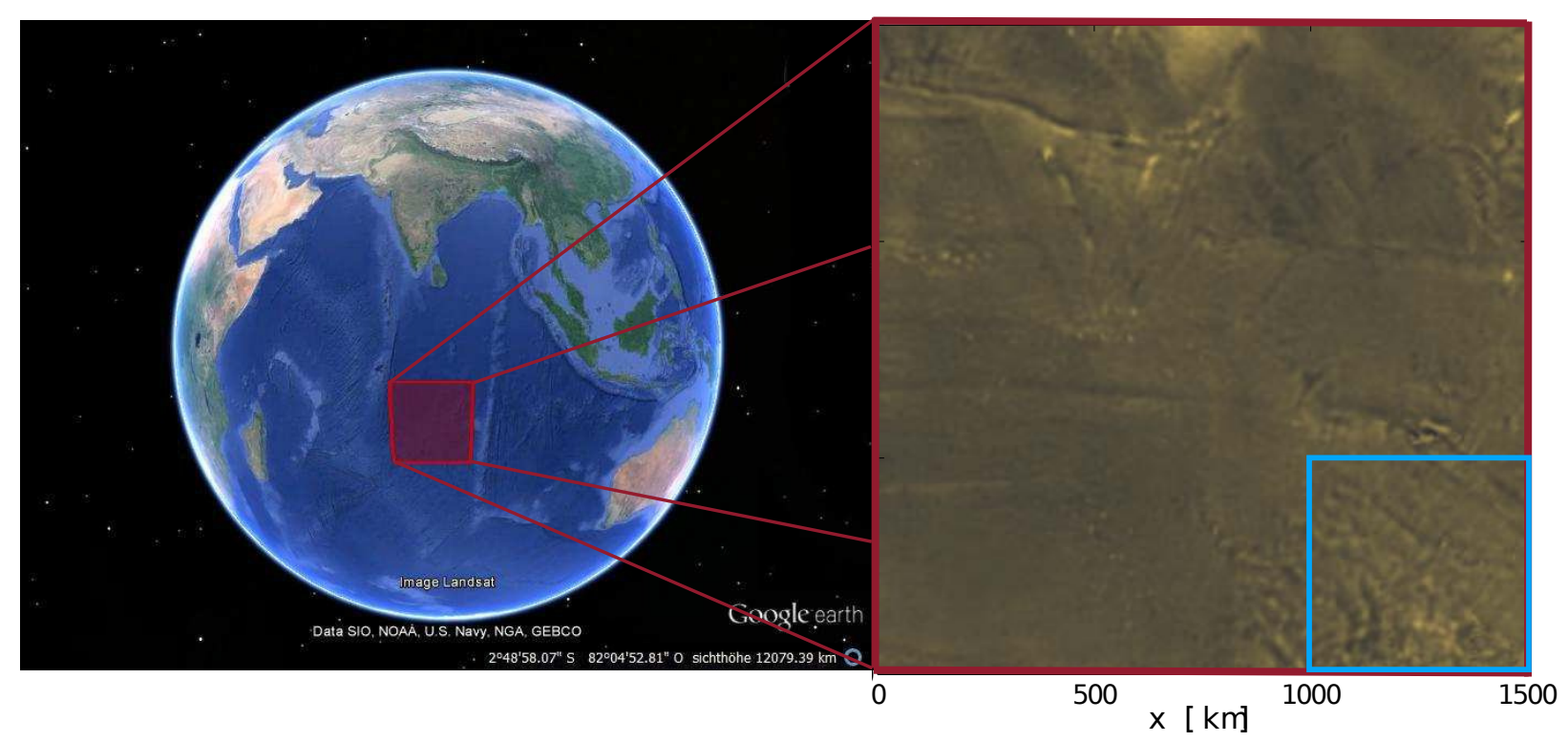

Figure 4.1: The right panel shows the ocean floor topography of the highlighted region (in the red square) on the Earth, on the left panel. The coordinates of this region ranges from $8^{\circ} 20^{\prime} \mathrm{S}$ to $20^{\circ} 50^{\prime} \mathrm{S}$ in latitude and from $73^{\circ} 20^{\prime} \mathrm{E}$ to $85^{\circ} 50^{\prime} \mathrm{E}$ in longitude. The blue square in the bottom right corner relates to Fig,4.2, Sources: Google Earth and GEBCO

Physically, a tsunami is typically produced by the relatively fast motion of a large portion of the ocean floor, the energy of which is transmitted to the surface of the ocean and excites surface waves. As the modeling of the impact of earthquakes on the water surface is a complicated and vast topic, we believe that it is out of the scope of this study and would actually not substantially contribute to our understanding of the focusing by small bathymetry structures. In all simulations, we will therefore model the source of the tsunami as a non-flat initial condition of the surface which then put the water in motion through the action of gravity. We do, however, show that our results do not rely on a particular choice of wave form for the source by studying different sources at the end of this chapter, see section 4.7. For our first numerical experiment, we model the source as an idealized point source. The geometry of real sources can be more complicated, which can lead to focusing on its own, as investigated in [7], but could be described by the superposition of many point sources. We use a model-source function that has a definite wavelength and is inspired by quantum mechanics, as presented in [65]. Our model use the following expression

$$
\psi(r)=\frac{e^{-r^{2} /\left(2 a^{2}\right)} e^{a^{2} k_{0}^{2} / 4}}{a \sqrt{\pi I_{0}\left(a^{2} k_{0}^{2} / 2\right)}} J_{0}\left(k_{0} r\right),
$$

where $r=\sqrt{x^{2}+y^{2}}, k_{0}=2 \pi / \lambda$ is the norm of the wave vector with the wavelength $\lambda, a$ is a constant scaling factor and $I_{0}$ and $J_{0}$ are Bessel functions. We chose a wavelength $\lambda=20 \mathrm{~km}$ for the sake of spatial resolution, because it turned out to be very complicated to find a region big enough that still fitted the requirements in terms of absence of islands and weak fluctuations that were discussed above. Nevertheless, we also verified that the same kind of fluctuations still appear with larger wavelengths (typically, 100km), as stronger earthquakes tend to produce tsunamis 


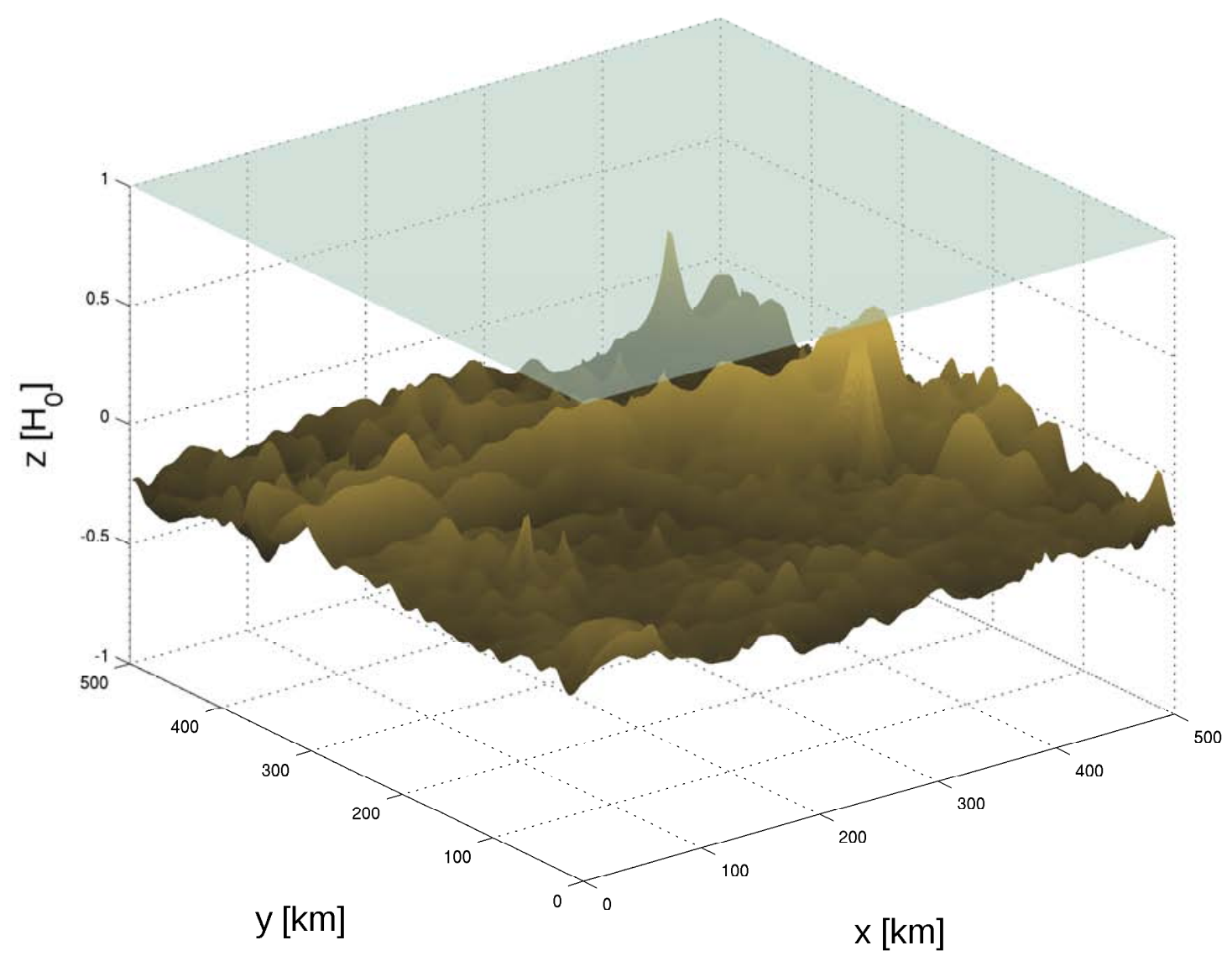

Figure 4.2: A 3D view of the ocean floor in the blue square of Fig 4.1. The vertical scale is magnified in order to see the variations. The unit on the z-axis means that the scale is given in multiples of the average depth $H_{0}=4.19 \mathrm{~km}$, i.e. the plane at $z=1$ represents the surface of the ocean and $z=0$ is the average depth. Source: GEBCO

with larger wavelength, which are therefore of higher interest due to their potentially greater destructive power. The figure corresponding to the $100 \mathrm{~km}$ wavelength is presented in section 4.7. In order to quantify the outcome of such a simulation, we need to define a good measure of the tsunami strength. Inspired by quantum mechanics, we use a time integrated intensity that we define as

$$
I(\vec{x})=\int_{0}^{T}[\eta(\vec{x}, t)]^{2} \mathrm{~d} t,
$$

where $\eta(\vec{x}, t)$ is the wave height and the tsunami is excited at time $t=0$ and the upper integration limit $t=T$ is chosen such that the wave has left the region of interest (i.e. we neglect reflections, e.g. from the coasts of continents, that are returning at longer times, which is reasonable in all shown examples). $I(\vec{x})$ is a measure of the potential energy of the wave that has been propagating through $\vec{x}$. Because we can, to good accuracy, assume equipartition of kinetic and potential energy of the propagating tsunami wave [66], $I(\vec{x})$ is an adequate measure of the total energy flux in $x$.

The result of the simulation can be seen in Fig. 4.3 and we clearly see the high intensity fluctuations typical of branched flow, with waves focused up to five times the average intensities. As we have now shown that tsunami waves do exhibit branched flow, we want to develop a general theory that describes the appearance of random caustics and therefore strongly focused waves. Since this phenomenon is random in its nature, we cannot produce any general argument 

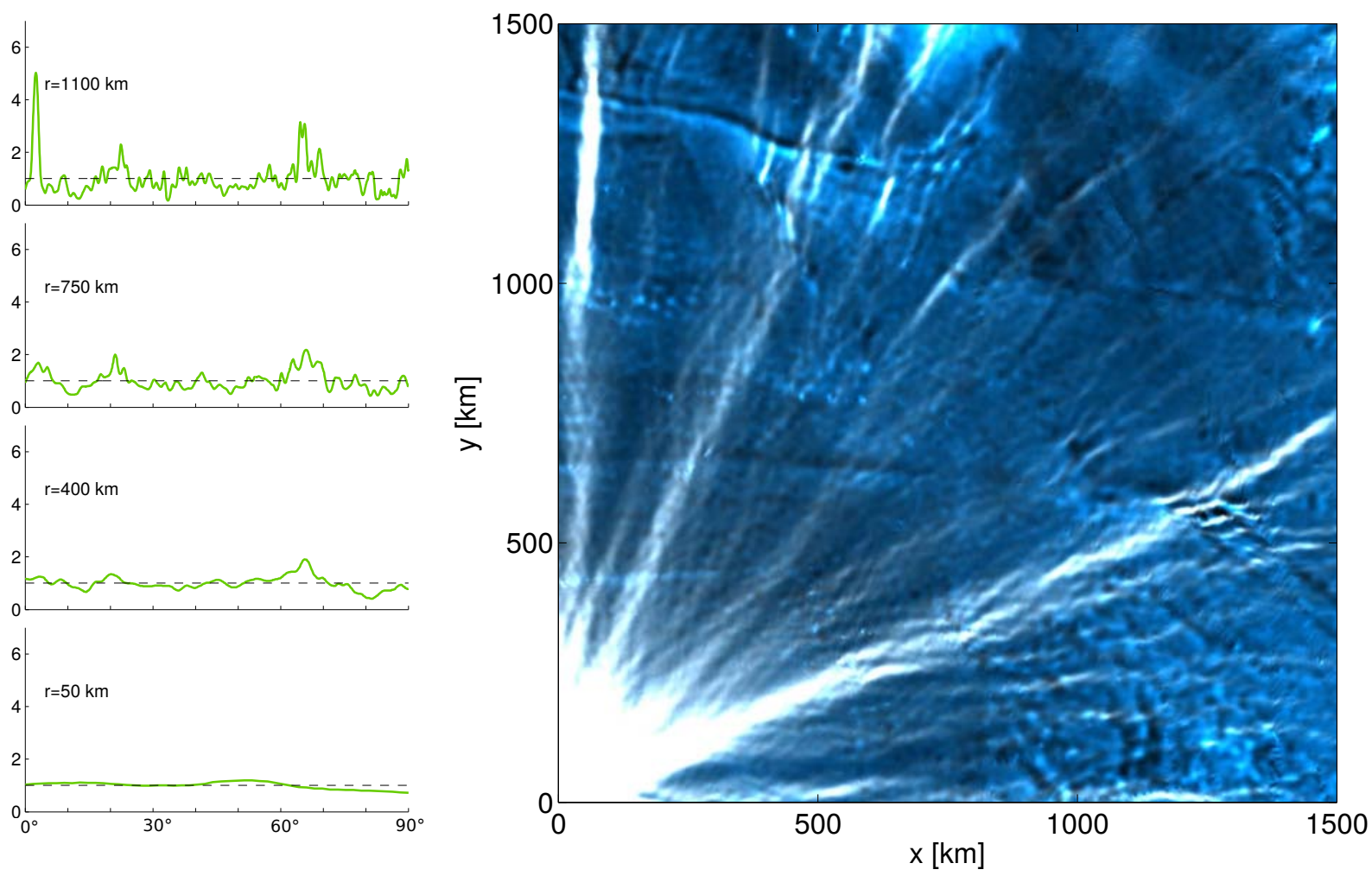

Figure 4.3: On the right panel, we can see the integrated intensity of a tsunami event propagated over the region of the ocean floor shown in Fig 4.1. We can clearly see the typical intensity fluctuations in branching patterns (the whiter the higher). On the left panel, we show several cuts in the intensity, which are made at various distances $r$ from the source at the south-western corner of the region. Each cut is then normalized by its own mean, such that we do not have to worry about the approximate $1 / r$ decay inherent to point source propagation. We can clearly see the emergence of strong branches, that can grow as high as five times the local mean after $1100 \mathrm{~km}$ of propagation.

by studying the system in a few specific bathymetries taken from the data base. We will thus study the ray equations related to tsunami propagation with $\beta(\vec{x})$ as a correlated random field, in order to obtain statistical quantities that will depend only on a small number of parameters that allow us to model a large variety of bathymetries.

\subsection{Stability analysis and caustics}

In chapter 2, we presented a method to study the occurrence of caustics using the properties of the curvature of the action in the so-called quasi-2D approximation, described in section 2.5 . Thanks to the fairly simple structure of the stochastic equations, we were able to calculate the mean time to the first caustics analytically, showing a simple scaling law, given in Eq.2.46. In the case of the ray equations associated with shallow water waves, however, the system cannot be described by a Hamiltonian, as is shown in appendix D. Because of this, we cannot directly apply the results shown in chapter 2 . There is, however, the possibility to use a quantity that would be the equivalent of this curvature of the action, even though there won't be an associate Hamilton-Jacobi equation. Indeed, we can use the simple definition from Eq. 2.5

$$
u=\frac{\partial p}{\partial x},
$$

to write $u$ in terms of the elements of the stability matrix $M(t)$, which is always well-defined. We develop this argument in one dimension for the sake of simplicity. Considering that, for a phase 
space element $\delta \vec{\phi}(t)=(\delta x(t), \delta p(t))$, the time evolution is given by

$$
\delta \vec{\phi}(t)=M(t) \delta \vec{\phi}(0)
$$

we can write

$$
\begin{aligned}
& \delta x(t)=M_{11}(t) \delta x(0)+M_{12}(t) \delta p(0), \\
& \delta p(t)=M_{21}(t) \delta x(0)+M_{22}(t) \delta p(0) .
\end{aligned}
$$

With these, the curvature $u(t)$ can be written as

$$
u(t)=\frac{\delta p(t)}{\delta x(t)}=\frac{M_{21}(t) \delta x(0)+M_{22}(t) \delta p(0)}{M_{11}(t) \delta x(0)+M_{12}(t) \delta p(0)}=\frac{M_{21}(t)+M_{22}(t) u(0)}{M_{11}(t)+M_{12}(t) u(0)} .
$$

Of course, it does not make so much sense to call it the curvature of the action anymore. We can, however, understand this quantity as the curvature of the equitemporal lines in phase space.

With this in mind, we see that we will need to compute the stability matrix elements in order to pursue this computation. We start from the ray equation derived in the previous chapter

$$
\begin{aligned}
\frac{d}{d t} \vec{x} & =(1-\beta(\vec{x})) \vec{p}, \\
\frac{d}{d t} \vec{p} & =\frac{c_{0}^{2} \nabla \beta(\vec{x})}{2(1-\beta(\vec{x}))}
\end{aligned}
$$

where $\beta(\vec{x})$ is the reduced bathymetry and will be considered as a weakly scattering, correlated, random field. For now, we will only look into bathymetries that are only correlated on a single length scale, called the correlation length $l_{c}$. We will extend the results to multiple-length-scale media in chapter 5 . It is interesting to notice that, compared to classical Newtonian rays that are used in chapter 2 , shallow water rays exhibit a multiplicative noise term $-\vec{p} \beta(\vec{x})$ in Eq.(4.8). This complication will actually prevent us from solving the mean first passage time problem. However, we believe it is worth showing where the method fails and it is still useful to go through it, as it will lead us to compute useful quantities, such as the stability matrix elements that will be used to locate the caustics numerically.

Even though we will derive the analytical result in the quasi-2D approximation, therefore using only the one-dimensional ray equations, we will compute the two-dimensional stability matrix, because we need its elements to find the caustics numerically, according to the caustic conditions described in section 2.3. As we cannot find a Hamiltonian for these ray equations, we cannot derive a Hamilton-Jacobi equation and we need to find another way to obtain an equivalent to the curvature equation. As we have shown above, we will need the stability matrix in order to find the curvature of the equitemporal lines. We expand the ray equations Eq.4.8 with infinitesimal displacement in phase space in order to find the equation for the the time evolution of the stability matrix. We call the phase space variable $\vec{\phi}=\left(x_{1}, x_{2}, p_{1}, p_{2}\right)^{T}$ and we expand them with $\vec{\phi} \rightarrow \vec{\phi}+\delta \vec{\phi}$, which gives, once inserted in the ray equations Eq.(4.8),

$$
\begin{aligned}
\dot{\vec{x}}+\delta \dot{\vec{x}} & =(1-\beta) \vec{p}-\vec{p}(\delta \vec{x} \cdot \nabla) \beta+(1-\beta) \delta \vec{p}+\mathcal{O}(\delta \vec{x} \cdot \delta \vec{p}), \\
\dot{\vec{p}}+\delta \dot{\vec{p}} & =\frac{c_{0}^{2} \nabla \beta(\vec{x})}{2(1-\beta(\vec{x}))}-c_{0}^{2} \delta \vec{x} \cdot\left[\frac{\nabla(\nabla \beta)}{2(1-\beta)}-\nabla \beta \frac{\nabla \beta}{2(1-\beta)^{2}}\right]+\mathcal{O}(\delta \vec{x} \cdot \delta \vec{x}),
\end{aligned}
$$

where we omitted the dependencies, i.e. $\beta=\beta(\vec{x})$. The first terms on each side of the two above equations are the original ray equations Eq. 4.8) and therefore cancel each other. We neglect the nonlinear terms $\mathcal{O}\left(\delta \phi_{i} \delta \phi_{j}\right)$. With this, we find the matrix $K(t)$ that will evolve the stability matrix

$$
\left(\begin{array}{l}
\dot{\delta} \vec{x} \\
\delta \vec{p}
\end{array}\right)=\left(\begin{array}{cc}
-\mathbb{G} & (1-\beta) \mathbb{I} \\
\frac{c_{0}^{2}}{2(1-\beta)} \mathbb{B} & 0
\end{array}\right)\left(\begin{array}{l}
\delta \vec{x} \\
\delta \vec{p}
\end{array}\right)=: K(t)\left(\begin{array}{l}
\delta \vec{x} \\
\delta \vec{p}
\end{array}\right)
$$


where we defined

$$
\mathbb{G}_{i j}=p_{i} \partial_{j} \beta ; \mathbb{B}_{i j}=\partial_{i j} \beta-\frac{\partial_{i} \beta \partial_{j} \beta}{(1-\beta)} ; i, j=1,2,
$$

and $\mathbb{I}$ is the two dimensional identity matrix.

The stability matrix $M$ is defined as the matrix that evolves the infinitesimal phase-space elements in time

$$
\delta \vec{\phi}(t)=M \delta \vec{\phi}(0)
$$

and is itself evolved in time by the matrix $K(t)$ found above

$$
\dot{M}(t)=K(t) M(t) .
$$

Indeed, when taking the time derivative of Eq.(4.12), we find

$$
\dot{\delta \vec{\phi}}(t)=\dot{M}(t) \delta \vec{\phi}(0) \text {. }
$$

The 1.h.s is then given by Eq.4.10, which leads to

$$
\dot{M}(t) \delta \vec{\phi}(0)=K(t) \delta \vec{\phi}(t)=K(t) M(t) \delta \vec{\phi}(0),
$$

from which we can deduce the matrix equation Eq.44.13), which will be used in the condition for a caustic in the shallow water ray simulations, where we solve numerically the stability matrix ODEs and use the conditions from section 2.3. The result is illustrated in Fig.4.5.

In order to investigate analytically the average time to the first caustics, we will again work in the quasi-2D approximation presented in section 2.5 , therefore using the one-dimensional version of this matrix

$$
\left(\begin{array}{l}
\dot{\delta x} \\
\delta p
\end{array}\right)=\left(\begin{array}{cc}
-p \beta^{\prime} & (1-\beta) \\
\frac{c_{0}^{2}}{2(1-\beta)}\left[\beta^{\prime \prime}-\frac{\left(\beta^{\prime}\right)^{2}}{1-\beta}\right] & 0
\end{array}\right)\left(\begin{array}{l}
\delta x \\
\delta p
\end{array}\right),
$$

where now the prime denotes the derivative in space (i.e. transverse direction). We know from Eq.4.13 that this matrix evolves the stability matrix in time, leading to the following equations of motion for the stability matrix elements

$$
\left(\begin{array}{ll}
M_{11} & M_{12} \\
M_{21} & M_{22}
\end{array}\right)=\left(\begin{array}{cc}
-p \beta^{\prime} & (1-\beta) \\
\frac{c_{0}^{2}}{2(1-\beta)}\left[\beta^{\prime \prime}-\frac{\left(\beta^{\prime}\right)^{2}}{1-\beta}\right] & 0
\end{array}\right)\left(\begin{array}{ll}
M_{11} & M_{12} \\
M_{21} & M_{22}
\end{array}\right) .
$$

As in chapter 2 , we will approximate the medium terms by white noise, and this for $\beta, \beta^{\prime}$ and $\beta^{\prime \prime}$ reads

$$
\beta^{(i)} \longrightarrow \sigma_{i} \Gamma_{i}(t)
$$

with

$$
\sigma_{i}^{2}=\left.\frac{(-1)^{i}}{2 c_{0}} \int_{-\infty}^{+\infty} d x\left[\partial_{y}^{2 i} c(x, y)\right]\right|_{y=0}
$$

and the $\Gamma$ 's are white noise with

$$
\left\langle\Gamma_{i}(t) \Gamma_{j}\left(t^{\prime}\right)\right\rangle=2 \delta_{i j} \delta\left(t-t^{\prime}\right) .
$$

For now, we will consider isotropic correlation functions that have a single length scale $l_{c}$ and can be written as

$$
c(x, y)=\epsilon^{2} f\left(\frac{x^{2}+y^{2}}{l_{c}^{2}}\right),
$$


where $\epsilon=\sqrt{\left\langle\beta^{2}\right\rangle}$ will be the standard deviation of the bathymetry generated by $c(x, y)$ and is assumed to be very small, i.e. $\epsilon \ll 1$. The function $f: \mathbb{R} \longrightarrow \mathbb{R}$, with $f(0)=1$, can be very general, as long as it is four times differentiable and that the integrals

$$
\kappa_{i}:=\int_{-\infty}^{+\infty} d z f^{(i)}\left(z^{2}\right)
$$

are convergent. Commonly used correlation functions, such as power laws and Gaussians, respect these conditions and the integrals are given in Tab 4.1. Typically, a Gaussian correlation function

\begin{tabular}{|c||c|c|c|}
\hline & $\kappa_{0}$ & $\kappa_{1}$ & $\kappa_{2}$ \\
\hline \hline$f(x)=e^{-x}$ & $\sqrt{\pi}$ & $-\sqrt{\pi}$ & $\sqrt{\pi}$ \\
\hline$f(x)=(1+x)^{-\nu}$ & $\mathcal{I}(\nu)$ & $-\nu \mathcal{I}(\nu+1)$ & $\nu(\nu+1) \mathcal{I}(\nu+2)$ \\
\hline$f(x)=\cosh ^{-1}(\sqrt{x})$ & $\pi$ & -1.16624 & 0.76548 \\
\hline
\end{tabular}

Table 4.1: This table contains the expressions for the values of the $\kappa_{i}$ with Gaussian and power law correlation functions. Here $\nu>\frac{1}{2}$ and $0<\mathcal{I}(\nu)=\int_{\mathbb{R}} d z\left(1+z^{2}\right)^{-\nu}=\sqrt{\pi} \frac{\Gamma\left(-\frac{1}{2}+\nu\right)}{\Gamma(\nu)}<\infty, \forall \nu>\frac{1}{2}$, where we use the $\Gamma$-function.

is described with $f(r)=e^{-r}$. With this defintions, we find

$$
\begin{aligned}
\sigma_{0}^{2} & =\frac{\epsilon^{2} l_{c}}{2 c_{0}} \kappa_{0}, \\
\sigma_{1}^{2} & =-\frac{\epsilon^{2}}{l_{c} c_{0}} \kappa_{1}, \\
\sigma_{2}^{2} & =\frac{6 \epsilon^{2}}{l_{c}^{3} c_{0}} \kappa_{2} .
\end{aligned}
$$

Now that our approximation for the $\beta^{(i)}$ 's is well-defined, we just need to insert their new definitions into Eq. 4.17). Unfortunately, we have the problematic terms

$$
\frac{\beta^{\prime \prime}}{2(1-\beta)}-\frac{\left(\beta^{\prime}\right)^{2}}{2(1-\beta)^{2}} \longrightarrow \frac{\sigma_{2} \Gamma_{2}}{2\left(1-\sigma_{0} \Gamma_{0}\right)}-\frac{\left(\sigma_{1} \Gamma_{1}\right)^{2}}{2\left(1-\sigma_{0} \Gamma_{0}\right)^{2}} .
$$

We have to deal with fractions of white noise. Our way around this is to consider that, as $\epsilon \longrightarrow 0$, the combination $\sigma_{0} \Gamma_{0} \sim \epsilon$ is also very small. Indeed, when we look at Eqs.(4.23), we see that $\sigma_{0}^{2}$ depends on a time scale given by $\tau_{c}:=l_{c} / c_{0}$, which is the time that the wave needs to propagate over one correlation length $l_{c}$ and is assumed to be smaller than our typical time scale $t$ in order for us to be able to approximate the random field by a white noise (see the quasi-2D approximation, section 2.5). We can therefore expand the fractions as

$$
\frac{\sigma_{2} \Gamma_{2}}{2\left(1-\sigma_{0} \Gamma_{0}\right)}-\frac{\left(\sigma_{1} \Gamma_{1}\right)^{2}}{2\left(1-\sigma_{0} \Gamma_{0}\right)^{2}} \simeq \frac{1}{2} \sigma_{2} \Gamma_{2}\left(1+\sigma_{0} \Gamma_{0}+\mathcal{O}\left(\sigma_{0}^{2} \Gamma_{0}^{2}\right)\right)-\frac{1}{2}\left(\sigma_{1} \Gamma_{1}\right)^{2}\left(1+2 \sigma_{0} \Gamma_{0}+\mathcal{O}\left(\sigma_{0}^{2} \Gamma_{0}^{2}\right)\right)
$$

As $\sigma_{2} \sim \sigma_{1} / l_{c} \ll \sigma_{1} \sim \sigma_{0} / l_{c} \ll \sigma_{0}$, we can neglect all the terms $\mathcal{O}\left(\sigma_{i} \sigma_{j} \Gamma_{i} \Gamma_{j}\right)$ and insert the result in the equations for the stability matrix elements $M_{i j}$

$$
\begin{aligned}
& \dot{M}_{11}=-p \sigma_{1} \Gamma_{1} M_{11}+\left(1-\sigma_{0} \Gamma_{0}\right) M_{21}, \\
& \dot{M}_{12}=-p \sigma_{1} \Gamma_{1} M_{12}+\left(1-\sigma_{0} \Gamma_{0}\right) M_{22}, \\
& \dot{M}_{21}=\frac{c_{0}^{2}}{2} \sigma_{2} \Gamma_{2} M_{11}, \\
& \dot{M}_{22}=\frac{c_{0}^{2}}{2} \sigma_{2} \Gamma_{2} M_{12} .
\end{aligned}
$$


The first important thing to notice is that the equations for $M_{11}$ and $M_{21}$ are coupled with each other and decoupled from the other two variables $M_{12}$ and $M_{22}$, and vice versa. We note here that, according to Eq. 4.7), the curvature for the plane waves initial condition $(u(0)=0)$ is given by

$$
u_{p w}(t)=\frac{M_{21}(t)}{M_{11}(t)},
$$

and we have

$$
u_{p s}(t)=\frac{M_{22}(t)}{M_{12}(t)},
$$

for point source initial conditions, as we have $u(0)=\infty$. Looking at the extremely symmetric structure of Eqs. (5.16), those two cases have exactly the same expression (up to a swap of the variable names)! From now on, we are going to develop the argument for the plane wave case, keeping in mind that the result is also valid for point sources. We will therefore want to merge Eq. $5.16 \mathrm{a}$ ) and Eq. 5.16 c) together to find a stochastic differential equation (SDE) for $u(t)$.

Also, $M_{11}$ is also coupled to $p$, which is not a constant. Its stochastic dynamics can be derived from the ray equations by using the same transformation for $\beta$ and its derivatives as above and performing the same expansion in $\mathcal{O}\left(\sigma_{i} \sigma_{j} \Gamma_{i} \Gamma_{j}\right)$. This yields

$$
\dot{p}=\frac{c_{0}^{2}}{2} \sigma_{1} \Gamma_{1} .
$$

The SDE for the curvature $u$ can be obtained by differentiating its definition

$$
\dot{u}=\frac{d}{d t}\left(\frac{M_{21}}{M_{11}}\right)=\frac{M_{11} \dot{M}_{21}-\dot{M}_{11} M_{21}}{M_{11}^{2}},
$$

and using the Eqs.(5.16), yielding

$$
\dot{u}=\frac{c_{0}^{2}}{2} \sigma_{2} \Gamma_{2}+p u \sigma_{1} \Gamma_{1}-\left(1-\sigma_{0} \Gamma_{0}\right) u^{2} .
$$

The two equations Eq.4.29 and Eq.4.31 form a system of Langevin equations, which can be written in a more compact form (the summation convention applies)

$$
\dot{\xi}_{i}=h_{i}(\vec{\xi})+g_{i j}(\vec{\xi}) \Gamma_{j-1}(t),
$$

with $i=\{1,2\}, j=\{1,2,3\}$ (the index $j-1$ of the $\Gamma$ is due to the fact that we labeled them with $\{0,1,2\}) \cdot \vec{\xi}=(u, p)$ and the functions $h_{i}$ and $g_{i j}$ are given by

$$
h_{1}=-\xi_{1}^{2} ; h_{2}=0
$$

and

$$
\begin{aligned}
g_{11} & =\sigma_{0} \xi_{1}^{2} \\
g_{21} & =g_{23}=0 \\
g_{12} & =\sigma_{1} \xi_{1} \xi_{2} \\
g_{22} & =\frac{c_{0}^{2} \sigma_{1}}{2} \\
g_{13} & =\frac{1}{2} c_{0}^{2} \sigma_{2} .
\end{aligned}
$$

Such a system of Langevin equations can be associated with a Fokker-Planck equation, as described in appendix B. We will need to compute the drift and diffusion coefficients of the backward F-P equation in order to calculate the mean first passage time to the first caustics, as is 
already described in section 2.6 for classical rays. The drift coefficients $D_{i}^{(1)}$ and the diffusion coefficients $D_{i j}^{(2)}$ are generally given by 55

$$
\begin{aligned}
D_{i}^{(1)} & =h_{i}+g_{k j} \partial_{k} g_{i j}, \\
D_{i j}^{(2)} & =g_{i k} g_{j k},
\end{aligned}
$$

where once again the summation convention applies. In our case, the coefficients are

$$
\begin{aligned}
D_{1}^{(1)}(\vec{\xi}) & =-\xi_{1}^{2}+2 \sigma_{0}^{2} \xi_{1}^{3}+\sigma_{1}^{2} \xi_{2}^{2} \xi_{1}+\frac{1}{2} c_{0}^{2} \sigma_{1}^{2} \xi_{1} \\
D_{2}^{(1)} & =0 \\
D_{11}^{(2)}(\vec{\xi}) & =\sigma_{0}^{2} \xi_{1}^{4}+\sigma_{1}^{2} \xi_{2}^{2} \xi_{1}^{2}+\frac{1}{4} c_{0}^{4} \sigma_{2}^{2} \\
D_{12}^{(2)}(\vec{\xi}) & =D_{12}^{(2)}=\frac{1}{2} c_{0}^{2} \sigma_{1}^{2} \xi_{1} \xi_{2} \\
D_{22}^{(2)} & =\frac{1}{4} c_{0}^{4} \sigma_{1}^{2} .
\end{aligned}
$$

As explained in detail in section 2.6, the probability density for a first focusing to happen at time $t$ considering initial conditions $\vec{\gamma}=\left(u_{0}, p_{0}\right)$ is called $p_{f}(t \mid \vec{\gamma})$ and satisfies the backward F-P equations (see Eq.(Bx))

$$
\partial_{t} p_{f}(t \mid \vec{\gamma})=-D_{i}^{(1)}(\vec{\gamma}) \frac{\partial}{\partial \gamma_{i}} p_{f}(t \mid \vec{\gamma})+D_{i j}^{(2)}(\vec{\gamma}) \frac{\partial^{2}}{\partial \gamma_{i} \partial \gamma_{j}} p_{f}(t \mid \vec{\gamma})
$$

We are trying to find an equation for the mean time to the first caustics $\left\langle t_{c}(\vec{\gamma})\right\rangle$, which is defined by the expectation value of the time variable $t$ with the probability density $p_{f}$

$$
\left\langle t_{c}(\vec{\gamma})\right\rangle=\int_{-\infty}^{+\infty} \mathrm{d} t t p_{f}(t \mid \vec{\gamma}) .
$$

If we multiply Eq. (4.38) by $t$ and integrate, we will obtain a PDE for $\left\langle t_{c}(\vec{\gamma})\right\rangle$. As shown in section 2.6 (see Eq.2.41), the 1.h.s. will simply give -1 . When we insert the drift and diffusion coefficients computed in Eq. 4.37), we find

$$
\begin{aligned}
-1 & =-\left(2 \sigma_{0}^{2} \gamma_{1}^{3}-\gamma_{1}^{2}+\sigma_{1}^{2} \gamma_{1} \gamma_{2}^{2}+\frac{c_{0}^{2} \sigma_{1}^{2}}{2} \gamma_{1}\right) \frac{\partial}{\partial \gamma_{1}}\left\langle t_{c}(\vec{\gamma})\right\rangle \\
& +\left(\sigma_{0}^{2} \gamma_{1}^{4}+\sigma_{1}^{2} \gamma_{1}^{2} \gamma_{2}^{2}+\frac{1}{4} c_{0}^{4} \sigma_{2}^{2}\right) \frac{\partial^{2}}{\partial \gamma_{1}^{2}}\left\langle t_{c}(\vec{\gamma})\right\rangle \\
& +c_{0}^{2} \sigma_{1}^{2} \gamma_{1} \gamma_{2} \frac{\partial^{2}}{\partial \gamma_{1} \partial \gamma_{2}}\left\langle t_{c}(\vec{\gamma})\right\rangle+\frac{c_{0}^{4} \sigma_{1}^{2}}{4} \frac{\partial^{2}}{\partial \gamma_{2}^{2}}\left\langle t_{c}(\vec{\gamma})\right\rangle .
\end{aligned}
$$

This is the point where we have to stop this calculation, as this equation is an incredibly complicated nonlinear PDE which is most likely unsolvable. Further simplifications, like keeping only the strongest terms, would lead to non-physical solutions. We do not exclude, though, that solving this equation can be done, given some extra expertise and technique, and this would be a great addition to this work, as it would give us the missing analytical prefactor to the result we derive later and thus a complete analytical expression.

There is a detail worth mentioning, though. This equation, unlike its equivalent for classical rays, is a PDE that has not only the initial curvature $\gamma_{1}=u_{0}$ as an independent variable, but also the initial momentum variable $\gamma_{2}=p_{0}$. Even though it is possible that this extra dependency comes from the fact that this variable is directly related to the potential in its definition, this feature is 
definitely not present in the classical ray case. However, as we are mainly interested in two well-defined types of source, namely plane waves and point source, the inital transverse momentum will always be constant with value zero. As we will need to numerically estimate the prefactor for the scaling law later on, this dependency will be included in the prefactor we will find.

Of course, this setback is not going to stop us here. We will now use another approach in order to estimate the mean distance to the first caustics, based on an idea developed by Kaplan 22. Even though we will be able to find the scaling law for the distance to the first caustics, this method unfortunately does not allow us to find the exact prefactor.

\subsection{Scaling law}

The argument that will be used is illustrated in Fig 4.4 and goes as follows. A caustic occurs at a location where the Lagrangian manifold has a turning point, as explained in chapter 2. Therefore, the manifold must have a second fold, meaning that two trajectories cross at this point. The idea is that, to be able to cross, they must have experienced different forces along their respective paths, so their initial positions must have been separated by a distance on the order of the correlation length. We can flip this construction around by saying that, in order for a trajectory to reach its first caustic, it needs to have traversed at least one correlation length in the transverse direction. In order to formulate this argument, we want to quantify the displacement $\Delta y(t)=y(t)-y(0)$ of one ray in the transverse direction and compare it to the correlation length $l_{c}$ at the time of the first caustics $t_{f}$. We will use the one dimensional ray equations Eq. (4.8) in the quasi-2D approximation, thus replacing the reduced bathymetry $\beta$ and its derivative by white noise, as we did for the stability matrix equations, with Eqs.(4.18 4.23). This yields

$$
\begin{aligned}
\frac{d}{d t} y & =\left(1-\sigma_{0} \Gamma_{0}\right) p, \\
\frac{d}{d t} p & =\frac{c_{0}^{2} \sigma_{1} \Gamma_{1}}{2} .
\end{aligned}
$$

Even though the exact solution for $y(t)$ is not known, we can compute the moments, using the Fokker-Planck equation associated with the Langevin equations (4.41). Once again, we define the vector $\vec{\xi}=(y, p)$ to write the equations in a compact form

$$
\dot{\xi}_{i}=h_{i}(\vec{\xi})+g_{i j}(\vec{\xi}) \Gamma_{j-1}(t),
$$

with $i, j=\{1,2\}$ and the functions $h_{i}$ and $g_{i j}$ are given by

$$
h_{1}(\vec{\xi})=\xi_{2} ; h_{2}=0
$$

and

$$
\begin{aligned}
g_{11}(\vec{\xi}) & =-\sigma_{0} \xi_{2} \\
g_{12} & =g_{21}=0 \\
g_{22} & =\frac{1}{2} c_{0}^{2} \sigma_{1} .
\end{aligned}
$$

With the definitions given above in Eq.4.36), we find the drift and diffusion coefficients

$$
\begin{aligned}
D_{1}^{(1)}(\vec{\xi}) & =\xi_{2} \\
D_{2}^{(1)} & =0 \\
D_{11}^{(2)}(\vec{\xi}) & =\sigma_{0}^{2} \xi_{2}^{2} \\
D_{12}^{(2)} & =D_{12}^{(2)}=0 \\
D_{22}^{(2)} & =\frac{1}{4} c_{0}^{4} \sigma_{1}^{2} .
\end{aligned}
$$




\section{Quasi-2D phase space}

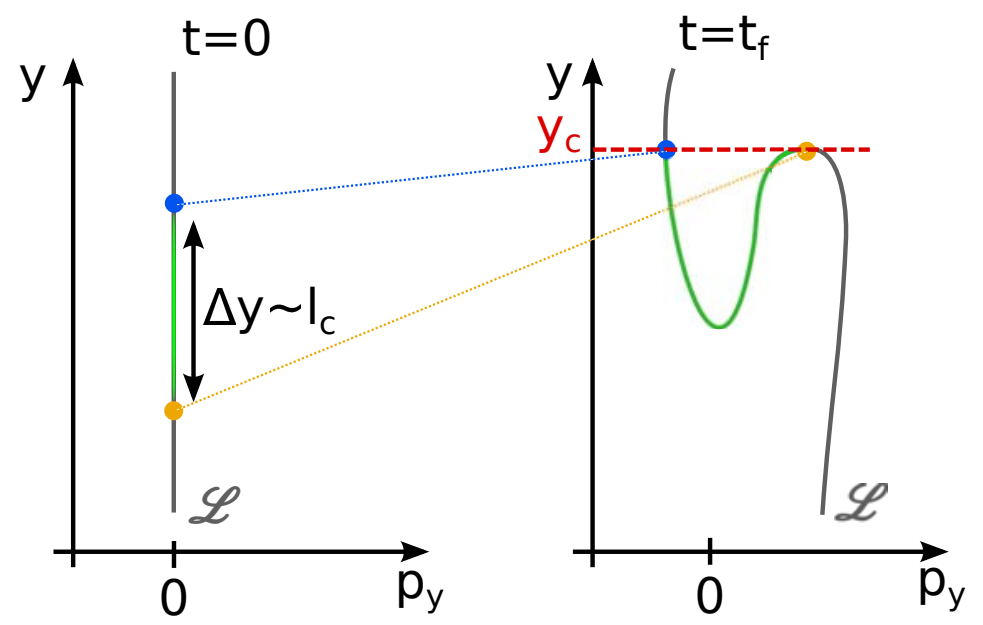

Position space

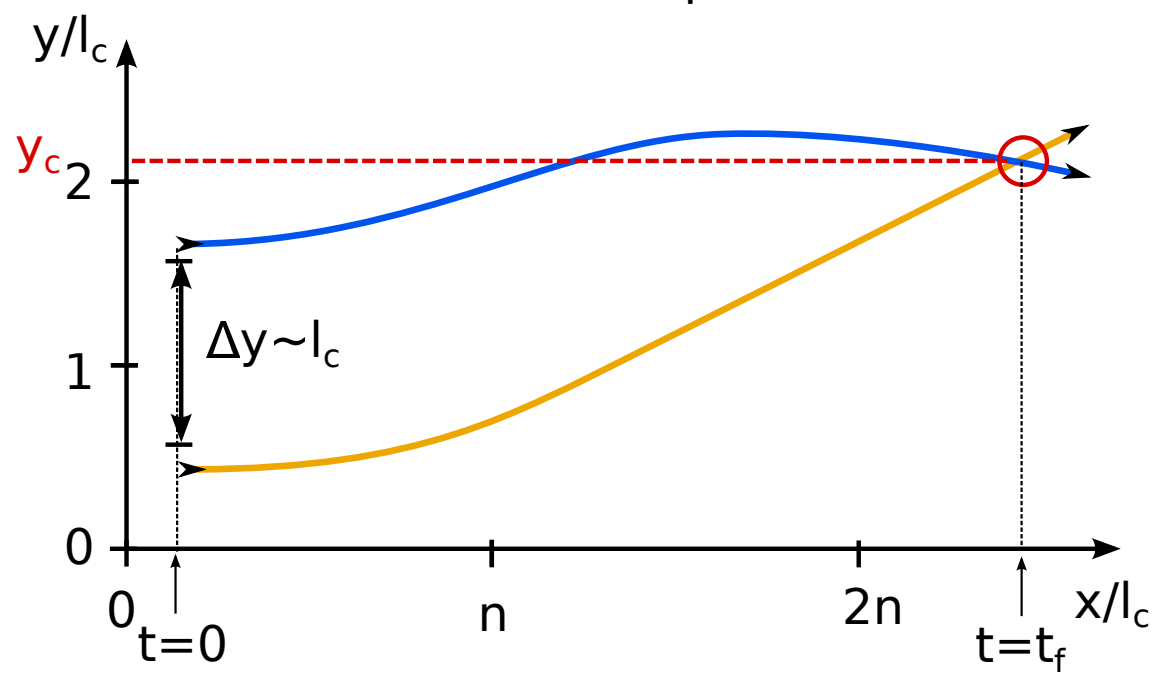

Figure 4.4: The upper part of the figure is presented in the phase space under the paraxial approximation, where we can see the Lagrangian manifold $\mathcal{L}$ at time $t=0$ on the left and at the time of the first caustic $t=t_{f}$ on the right. As we can see, at the moment of the caustic, one of the trajectories (the orange one) is at the turning point of the Lagrangian manifold, while the other trajectory (the blue one) is at the other value of the manifold for the same position in space $y_{c}$. Those points are separated by the green region along the Lagrangian manifold. In order for the two parts of the manifold to have such different dynamics, the forces they were subject to had to be significantly different along their paths, which means that the potential was therefore very different. Such difference can only occur on distances larger than the correlation length $l_{c}$. The lower panel shows the projection of this onto position space, where in the paraxial approximation $x \sim t$. Here, $n \gg 1$, which symbolizes the fact that the rays move much faster in the propagation direction.

Now, the probability density $P(t, \vec{\xi})$ satisfies the forward F-P equation (see appendix B) given by

$$
\partial_{t} P(t, \vec{\xi})=\left[-\frac{\partial}{\partial \xi_{i}} D_{i}^{(1)}+\frac{\partial^{2}}{\partial \xi_{i} \partial \xi_{j}} D_{i j}^{(2)}\right] P(t, \vec{\xi})
$$

which gives, with Eqs.4.45,

$$
\partial_{t} P(t, \vec{\xi})=-\xi_{2} \frac{\partial}{\partial \xi_{1}} P(t, \vec{\xi})+\sigma_{0}^{2} \xi_{2}^{2} \frac{\partial^{2}}{\partial \xi_{1}^{2}} P(t, \vec{\xi})+\frac{1}{4} c_{0}^{4} \sigma_{0}^{2} \frac{\partial^{2}}{\partial \xi_{2}^{2}} P(t, \vec{\xi}) .
$$


As the white noise terms are statistically symmetric, we can assume that the means $\langle y\rangle$ and $\langle p\rangle$ will be zero. We are therefore going to look at the variance, which will be given by the second moment

$$
\left\langle y^{2}(t)\right\rangle=\int_{\mathbb{R}^{2}} \mathrm{~d} y \mathrm{~d} p y^{2} P(t, y, p),
$$

where $P(t, y, p)$ satisfies the F-P equation Eq.4.47). We compute this by differentiating Eq. 4.48 with respect to time

$$
\frac{d}{d t}\left\langle y^{2}(t)\right\rangle=\frac{d}{d t} \int_{\mathbb{R}^{2}} \mathrm{~d} y \mathrm{~d} p y^{2} P(t, y, p)=\int_{\mathbb{R}^{2}} \mathrm{~d} y \mathrm{~d} p y^{2} \partial_{t} P(t, y, p),
$$

where the second equality comes from the fact that the boundaries do not depend on time. Using the forward F-P equation Eq.4.47) in the integral, we obtain

$$
\frac{d}{d t}\left\langle y^{2}(t)\right\rangle=\int_{\mathbb{R}^{2}} \mathrm{~d} y \mathrm{~d} p y^{2}\left[-p \partial_{y}+p^{2} \partial_{y}^{2}+\frac{1}{4} c_{0}^{4} \sigma_{1} \partial_{p}^{2}\right] P(t, y, p) .
$$

We can integrate each term by parts, yielding

$$
\begin{aligned}
\int_{\mathbb{R}^{2}} \mathrm{~d} y \mathrm{~d} p y^{2} p \partial_{y} P & =-\int_{\mathbb{R}} \mathrm{d} p p\left[\left.y^{2} P\right|_{-\infty} ^{+\infty}-2 \int_{\mathbb{R}} \mathrm{d} y y P\right] \\
& =2 \int_{\mathbb{R}^{2}} \mathrm{~d} y \mathrm{~d} p y p P=2\langle p y\rangle \\
\sigma_{0}^{2} \int_{\mathbb{R}^{2}} \mathrm{~d} y \mathrm{~d} p y^{2} p^{2} \partial_{y}^{2} P & =\sigma_{0}^{2} \int_{\mathbb{R}} \mathrm{d} p p^{2}\left[\left.y^{2} \partial_{y} P\right|_{-\infty} ^{+\infty}-2 \int_{\mathbb{R}} \mathrm{d} y y \partial_{y} P\right] \\
& =2 \sigma_{0}^{2} \int_{\mathbb{R}} \mathrm{d} p p^{2}\left[-\left.y P\right|_{-\infty} ^{+\infty}+\int_{\mathbb{R}} \mathrm{d} y P\right] \\
& =2 \sigma_{0}^{2} \int_{\mathbb{R}^{2}} \mathrm{~d} p \mathrm{~d} y p^{2} P=2 \sigma_{0}^{2}\left\langle p^{2}\right\rangle \\
\frac{1}{4} \sigma_{1}^{2} \int_{\mathbb{R}^{2}} \mathrm{~d} y \mathrm{~d} p y^{2} \partial_{p}^{2} P & =\left.\frac{1}{4} \sigma_{1}^{2} \int_{\mathbb{R}} \mathrm{d} y y^{2} \partial_{p} P\right|_{-\infty} ^{+\infty}=0,
\end{aligned}
$$

where the boundary terms have been considered to be zero because $P$ is a probability distribution which therefore vanishes at $\pm \infty$. Using Eqs. (4.51), we can write

$$
\frac{d}{d t}\left\langle y^{2}(t)\right\rangle=2\left[\langle p y(t)\rangle+\sigma_{0}^{2}\left\langle p^{2}(t)\right\rangle\right] .
$$

We can now find similar ODEs for $\langle p y(t)\rangle$ and $\left\langle p^{2}(t)\right\rangle$ using the same method, which brings

$$
\begin{aligned}
\frac{d}{d t}\langle p y\rangle & =\left\langle p^{2}\right\rangle, \\
\frac{d}{d t}\left\langle p^{2}\right\rangle & =\frac{1}{2} c_{0}^{4} \sigma_{1}^{2} .
\end{aligned}
$$

The system of ODEs formed by Eqs.4.52 4.53 is closed and can be integrated, starting from Eq. $4.53 \mathrm{~b})$

$$
\left\langle p^{2}(t)\right\rangle=\frac{1}{2} c_{0}^{4} \sigma_{1}^{2} t+A .
$$


Since the initial variance is zero because of the initial conditions, $A=0$. With this, we can integrate $\langle p y(t)\rangle$, yielding (again, neglecting the integration constant for the same reasons)

$$
\langle p y(t)\rangle=c_{0}^{4} \sigma_{0}^{2} t^{2}
$$

which leads to

$$
\left\langle y^{2}(t)\right\rangle=c_{0}^{4}\left[\frac{1}{6} \sigma_{1}^{2} t^{3}+\frac{1}{2} \sigma_{1}^{2} \sigma_{0}^{2} t^{2}\right] .
$$

We say that, at the time of the first caustics $t_{f}$, the variance should be similar to the correlation length $l_{c}$ squared

$$
\left\langle y^{2}\left(t_{f}\right)\right\rangle \sim l_{c}^{2} .
$$

As $\sigma_{0}$ is very small, we can neglect the terms in $t^{2}$ in Eq. (4.56) and write

$$
\frac{1}{6} c_{0}^{4} \sigma_{1}^{2} t_{f}^{3} \sim l_{c}^{2},
$$

which, using the definition of $\sigma_{1}$ in Eq. 4.23 , gives the average time to the first caustics

$$
t_{f} \sim-l_{c} \epsilon^{-2 / 3} c_{0}^{-1} \kappa_{1}^{-1 / 3} .
$$

As the propagation direction $x$ is proportional to time with $x=c_{0} t$, we find the distance to the first caustics $d_{f}$

$$
d_{f}=-C_{s}^{w}\left(\frac{6}{\kappa_{1}}\right)^{1 / 3} l_{c} \epsilon^{-2 / 3} .
$$

We note that the negative sign comes from the definition of $\sigma_{1}$ in Eq.4.23) and is canceled by the sign of $\kappa_{1}$, see table 4.1 for examples. We define $C_{s}^{w}$ as the geometrical factor for water rays that depends on the source $s$, which can be either for a plane wave $C_{p w}^{w}$ or a point source $C_{p s}^{w}$. Also, we note that we verified how good was the approximation from Eq.4.56) to Eq.4.58 by solving the third degree algebraic equation in $t$ and found that the error made by neglecting the term in $\sigma_{0}^{2} t^{2}$ is very small, on the order of $0.5 \%$ for $\epsilon=0.1$. We also note that all the properties of the correlation function lie in the factor $\kappa_{1}^{-1 / 3}$. Even though this kind of dependency was confirmed for classical rays in [37, we so far failed to verify it numerically for water rays. The prefactor found for another correlation function (see appendix E) was different by around $20 \%$. We cannot exclude that we omitted a numerical artifact, but it might also be a more profound effect, which would deserve more investigation. More details on this matter can be found in appendix E. Even though we do not have an exact analytic solution for the distance to the first caustics, as the prefactor is missing, we can still check that this scaling law is correct. In the next section, we will look at 2D simulations of the ray equations Eqs. (4.8) in random bathymetries with different standard deviations $\epsilon$ and correlation lengths $l_{c}$ in order to verify our theoretical scaling law. In the subsequent section, we will study the scaling numerically with wave simulations.

\subsection{Ray simulations}

We simulate the two-dimensional ray equations Eq. (4.8) on random bathymetries generated using the method shown in appendix A. We use a standard fourth-order Runge-Kutta solver to evolve the rays and stability matrix elements in time. We find the caustics using the stability matrix elements of the two-dimensional ray equations given in Eqs.4.17) and the conditions presented in section 2.3, in Eq. 2.19) and Eq. 2.22). The result of such a simulation can be seen in Fig.4.5, where the caustics are shown as they are detected by the above-mentioned conditions. First, we want to confirm that the scaling law we derived in Eq.44.60 is correct. We will therefore look at the mean distance to the first caustics for several hundred realizations of the potential, varying the standard deviation $\epsilon$ and the correlation length $l_{c}$. We can do this both for plane waves and point sources, therefore numerically establishing the prefactor of the scaling in those two cases. These results are shown in Fig 4.6 and Fig 4.7, where we show an excellent agreement between the simulations and the theory. All the simulations were done in Gaussian correlated bathymetries. 


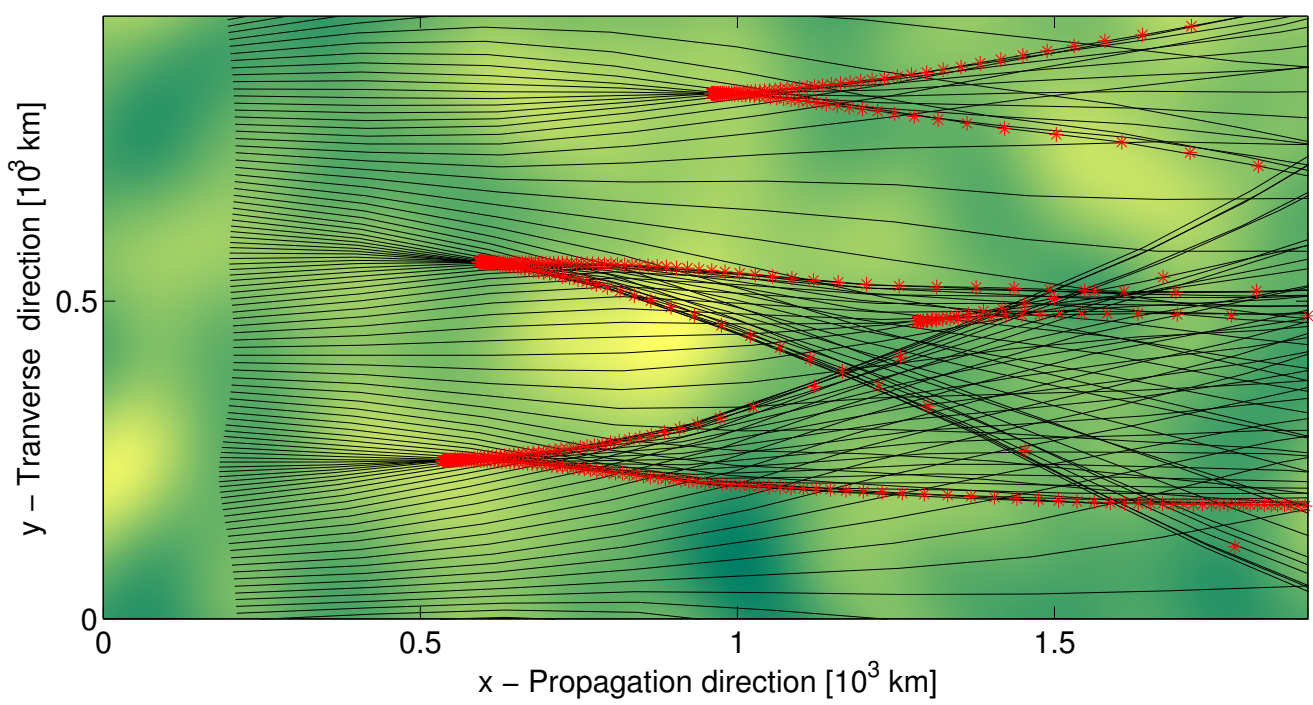

Figure 4.5: In black, we show ray trajectories (corresponding to a plane wave initial condition) propagating over a weakly scattering random potential which can be seen in the background with a green-yellow color map. Using the caustics conditions Eq. 2.19), we find the caustics, which are highlighted with red stars in this plot. We see that the caustics align in these characteristic branch-like structures and we clearly see the cusps and folds. When doing the statistics of the position of the first caustics, we stop the rays after the first caustic and we compute the mean distance traveled in the propagation direction until it is reached.

\subsubsection{Plane waves}

We started with plane wave initial conditions. We simulated tsunami rays with seven different values for $\epsilon$ and six different correlation lengths $l_{c}$ over 200 different randomly generated bathymetries. Then, we averaged the distance to the first caustics over all the rays and realizations of the random field with the same parameters. The results we obtained are presented in Fig.4.6. We can see on the right panel that all the points nicely collapse around the same line when we scale out the dependencies that we expected from Eq.4.60. These simulations were done using a Gaussian correlation function. If we scale out the other factors, i.e. the integral of the correlation function, we can determine the actual prefactor of our scaling law, which only depends on the geometry of the source. We find a prefactor of $C_{p w}^{w} \simeq 2.55$.

\subsubsection{Point source}

The point source statistics were done in a very similar fashion to the plane wave. The only changes are in the initial condition, that is now set to point source, which means that the distance has to be measured using the two-dimensional norm $d^{2}=x^{2}+y^{2}$. Of course, we expect a different prefactor, as in the classical ray case. The result can be seen in Fig 4.7 . We find $C_{p s}^{w} \simeq 3.78$

\subsection{Waves simulations}

Now that we have established that our analytical scaling law is valid for the two-dimensional rays, we need to verify that the random focusing of the tsunami waves actually follows the same scaling law. We will compare our analytical prediction to the occurence of the highest waves in shallow water simulations, where we will need to fit the prefactors, as the highest fluctuations in the intensity do not necessarily coincide with the first caustics, but should scale in the same way with the standard deviation and the correlation length. We used a finite-difference, leap-frog scheme to numerically integrate Eqs.(3.14 [67]. 

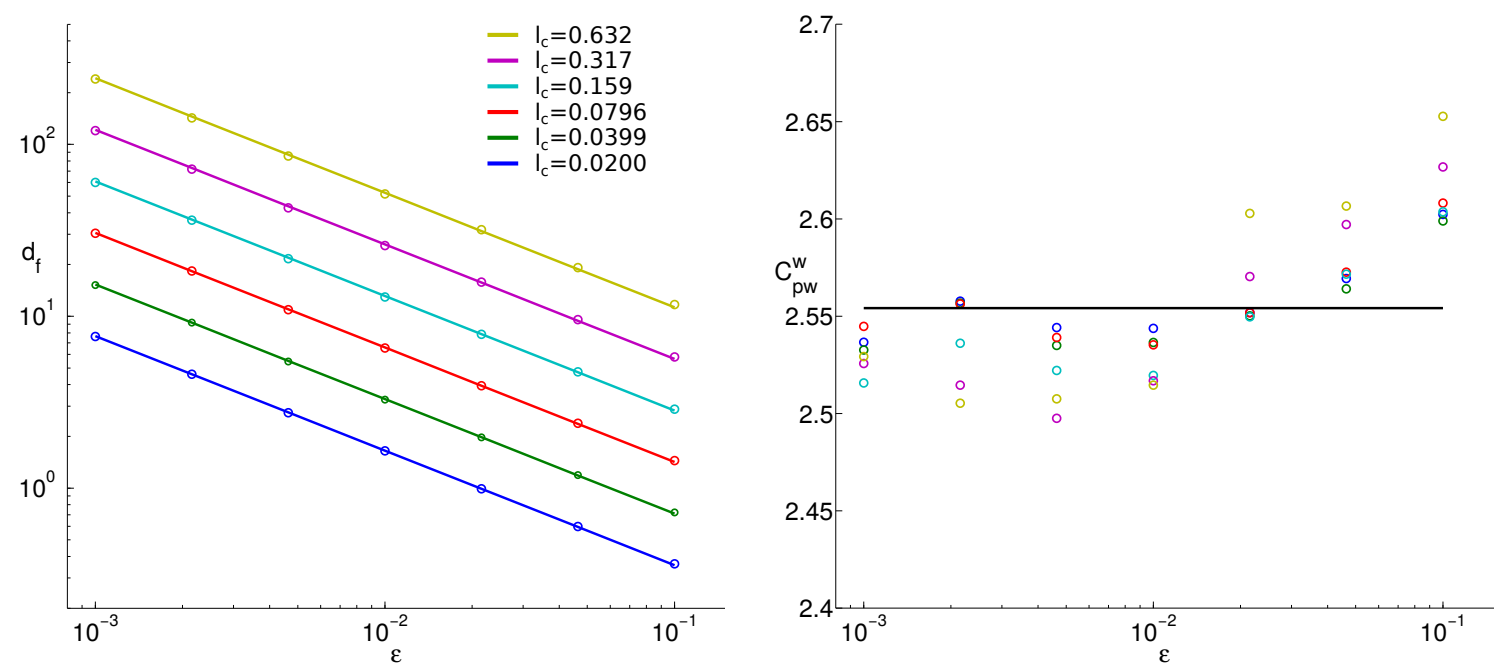

Figure 4.6: The left panel shows different theoretical lines in $\epsilon^{-2 / 3}$ for different values of $l_{c}$, which were fitted to the numerical data (dots) with the constant prefactor found on the right panel. We find that $C_{p w}^{w} \simeq 2.55$. The right panel shows how we obtained the missing prefactor in the theoretical prediction Eq.4.60 for plane waves: After we ran simulations with various values of $l_{c}$ and $\epsilon$ on several hundred bathymetries, we computed the average distance to the first caustics for each couple $\left(l_{c}, \epsilon\right)$. Each dot (in both panels) corresponds to one of these couples. We then divided the numerically obtained $d_{f}\left(l_{c}, \epsilon\right)$ by the theoretical value taken from Eq.4.60), without the prefactor $C_{p w}^{w}$. If our theory is correct, all the dots should collapse on the same value for the prefactor $C_{p w}^{w}$, which is the case here, up to small statistical fluctuations as all the points are in the window $2.55 \pm 0.1$. The units for the distance are arbitrary.
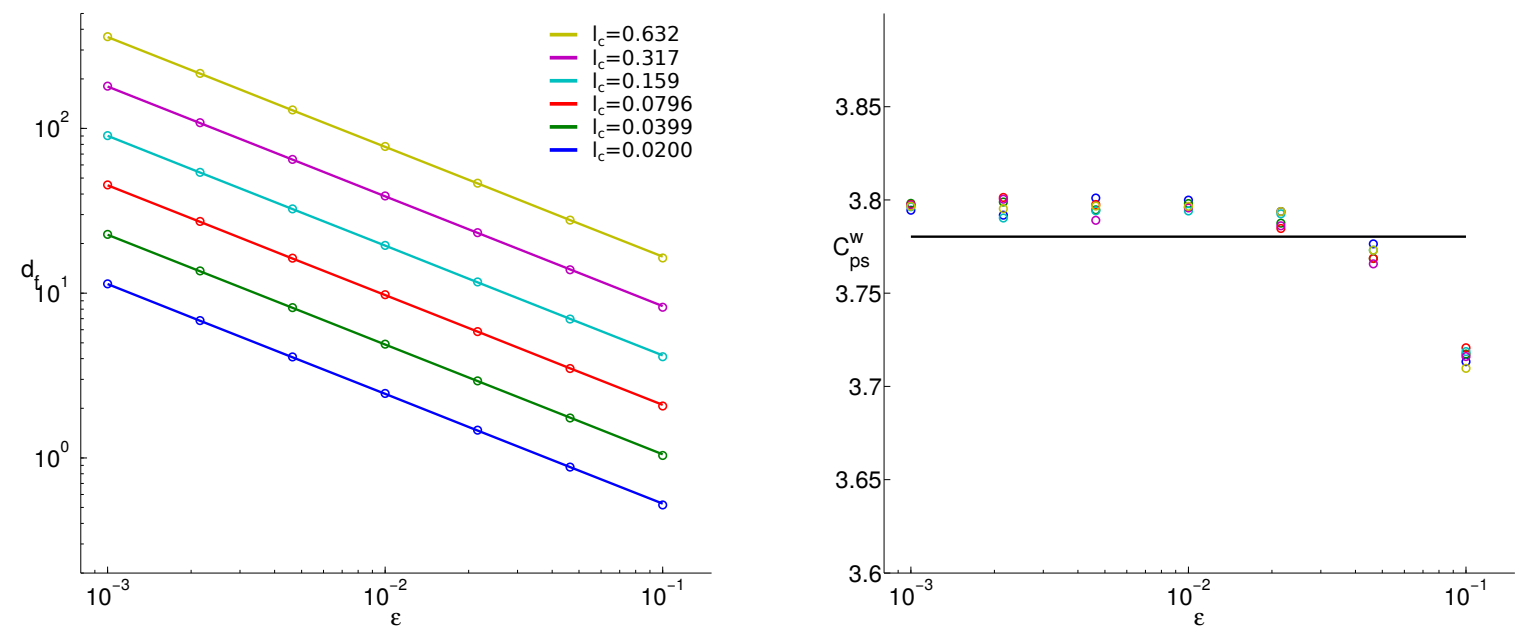

Figure 4.7: This plot was produced in the same way as Fig 4.6, but for point sources instead of plane waves. We find $C_{p s}^{w} \simeq 3.78$, with fluctuation on the order of 0.05 . The units for the distance are arbitrary.

The first problem that arises when dealing with wave simulations is that we do not have a well-defined mathematical condition for a caustic. The tool we will use to study the spatial dependences of the wave focusing numerically is called the scintillation index, which will be presented in the next section. 


\subsubsection{Scintillation index}

Caustics are classically defined as the coalescence of ray trajectories which can be very well described with the help of the stability matrix elements and the curvature of the action. These features are not easily translated into the wave picture. The simple fact that the ray density diverges in the classical case, unlike the wave intensity, shows that these descriptions do not compare well at caustics. It is then obvious that we need a new way to find the region of strong focusing.

Let us consider a tsunami wave propagating in the $x$ direction in a plane wave initial condition over a correlated random medium. The transverse direction will be called $y$. After some time, the wave is going to be focused in some locations (caustics) due to the branching of the flow. After that first generation of focusing, the wave will keep branching. Branches will overlap and interferences will start to dominate the intensity fluctuations, leading to the so-called saturated regime. This process can be seen in Fig 4.8. We want to find an efficient way to measure the strength of the intensity fluctuation in the wave flow. A good measure of this can be obtained by calculating the variance of the intensity $I(x, y)$ in the transverse direction and normalize it with the mean squared, in case there is decay, e.g. for point sources. This measure is called the scintillation index $\Sigma$ and can be written as

$$
\Sigma(x)=\frac{\left\langle I^{2}(x, y)\right\rangle_{y}-\langle I(x, y)\rangle_{y}^{2}}{\langle I(x, y)\rangle_{y}^{2}}
$$

where the index $y$ in the $k$-th moment indicates that it is taken along the transverse direction $y$

$$
\left\langle I^{k}(x, y)\right\rangle_{y}=\frac{1}{b-a} \int_{a}^{b} \mathrm{~d} y I^{k}(x, y) .
$$

Of course, this measure can also be written in polar coordinate for point sources, where the propagation direction is $r$ and the tranverse direction is the angle $\theta$

$$
\Sigma(r)=\frac{\left\langle I^{2}(r, \theta)\right\rangle_{\theta}-\langle I(r, \theta)\rangle_{\theta}^{2}}{\langle I(r, \theta)\rangle_{\theta}^{2}}
$$

where, once again, the index $\theta$ in the $k$-th moment indicates that they are taken along the transverse direction $\theta$

$$
\left\langle I^{k}(r, \theta)\right\rangle_{\theta}=\frac{1}{2 \pi} \int_{-\pi}^{\pi} \mathrm{d} \theta I^{k}(r, \theta) .
$$

As can be seen in Fig 4.8 , the scintillation index peaks at the first caustics, and then falls off to an asymptote with $\Sigma(\infty) \longrightarrow 1$ due to interferences. We will use this tools to estimate the distance to the first caustics for every simulation and compute the average location of the first peak, relating it to the distance to the first caustics.

\subsubsection{Scaling with respect to $\epsilon$}

As the wave simulations are significantly more demanding than their ray counterparts, we only ran them for one fixed correlation length and varied the standard deviation $\epsilon$, since, as long as the wavelength is much shorter than the correlation length, the role of the latter appears to consist in just setting a spatial scale (linear dependency). As the measure of the position of the caustics is the scintillation index, we note that our theoretical prediction should hold, but the prefactor found for rays is likely to be inaccurate, as the peak in the variance is not located at the first caustics. The results of our simulations are presented in Fig 4.9. We can see the excellent agreement of the scaling of the distance to the first peak with the prediction of $\epsilon^{-2 / 3}$. We realize in these scaling plots that even for tiny fluctuations in the bathymetry, of the order of a couple of percent, the distance to the strongest fluctuations is actually on the order of a couple of thousands of kilometers, which is very relevant for tsunami propagation. 

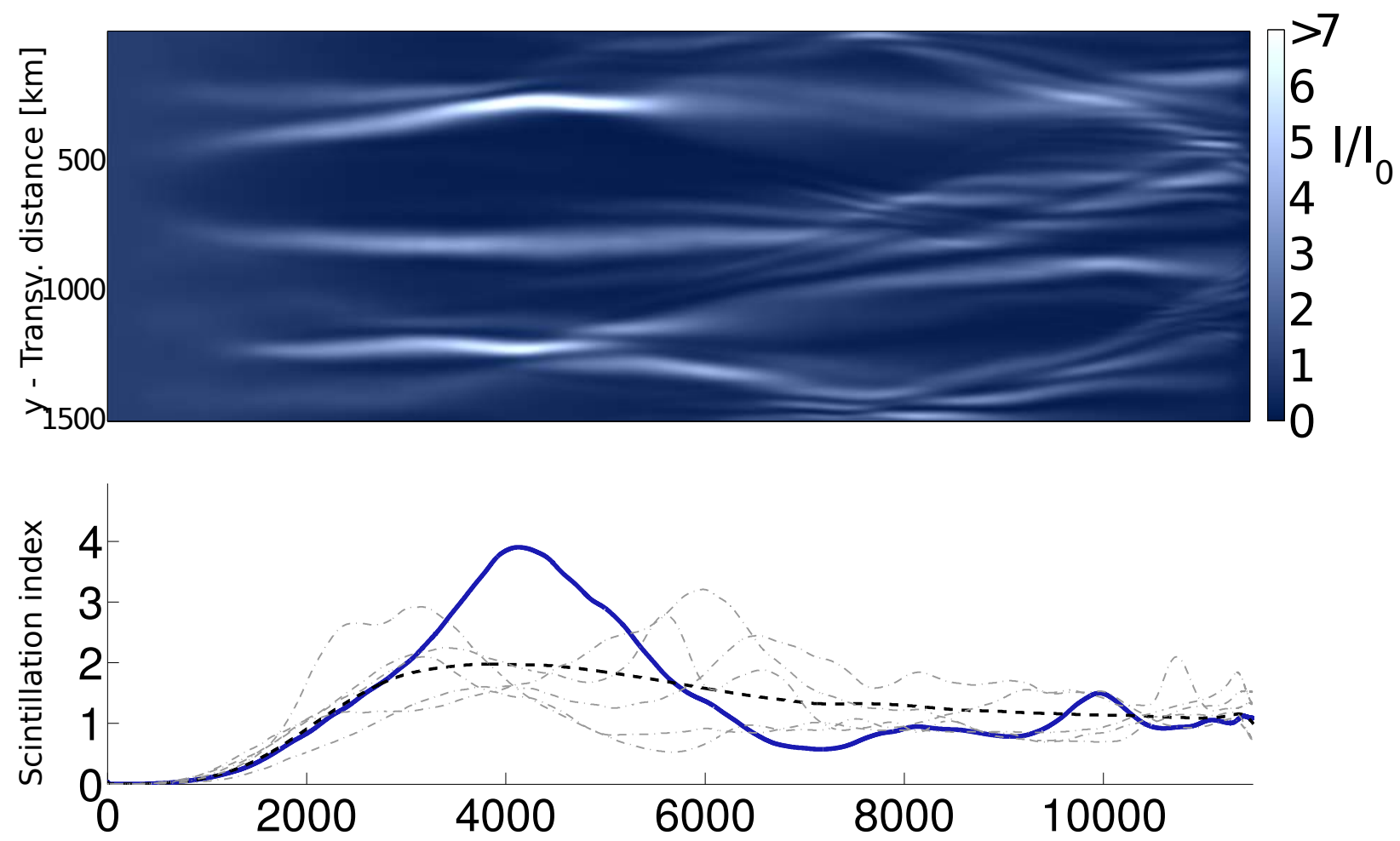

$\mathrm{x}$ - Propagation distance $[\mathrm{km}]$

Figure 4.8: The upper panel show the time-integrated intensity of a plane wave propagating from left to right over a random bathymetry (not shown), in a blue-white color map (see color bar). We divided the intensities by the mean intensity to show how strong the brightest branches are (more than seven times the mean). We can clearly see that the first branches are the most intense ones. Then, we enter the saturated regime, on the right of the plot. The bottom panel shows the corresponding scintillation index in blue, which peaks around the location of the first branches. In dashed black, one can see an average scintillation index, obtained by averaging the scintillation indices from two hundred realizations of the bathymetry. The dotted gray curves are examples of other realizations, for illustration purposes.

\subsection{Impact on tsunami forecasts}

With such a sensitivity to small fluctuations, concerns about the precision of the bathymetry measurements arise. We will show in this section that the current knowledge of the ocean floor topography is not accurate enough for reliable tsunami forecast due to the sensitivity of the branching patterns to the small fluctuation of the ocean floor. We will start by presenting the measurement methods used in order to map the bathymetry.

\subsubsection{Echo-sounding}

The first and most accurate method used to measure the ocean floor topography is echo-sounding. Ships, equipped with sonar, scan the ocean floor under their path and reference the measured values on a map, now using GPS location tracking. However, in more than five decades, we still lack more than $90 \%$ of coverage of the ocean floor by this method, see Fig 4.10 . It is estimated that a systematic survey of the ocean floor using this technique would take 200 ship-years and cost billions of dollars [68,69]. It appears that a complete mapping of the bathymetry using this method is not an option at the time of writing, both economically and time-wise. In order to solve this issue, a faster and cheaper method was developed, based on the 

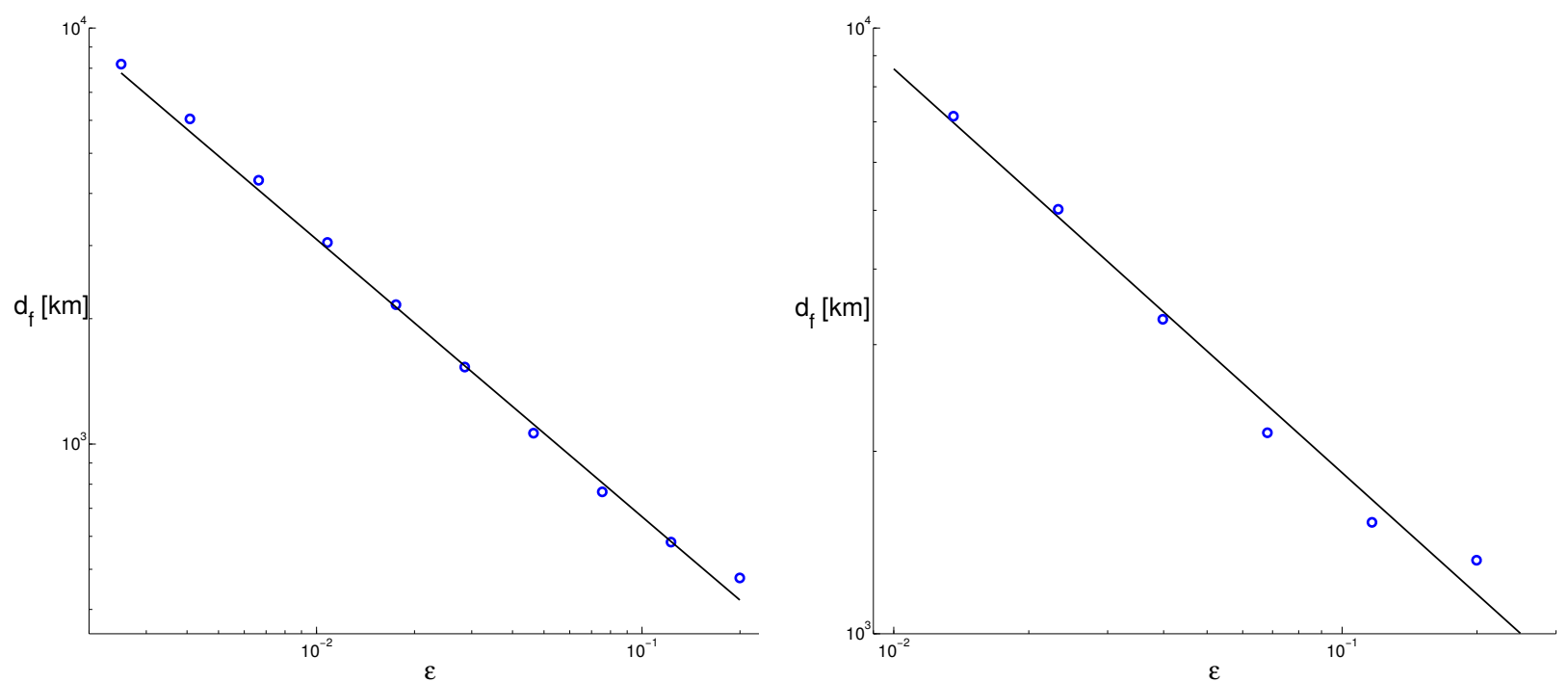

Figure 4.9: Here we have various numerical points (dots) for the average distance to the first peaks of the scintillation index, both for plane wave initial condtions (left) and point source (right). The theoretical prediction is fitted to the data with an extra prefactor of 0.7 . We start to see deviations from the theoretical line for the highest value of $\epsilon$, which is coming from the breaking of the quasi-2D approximation.

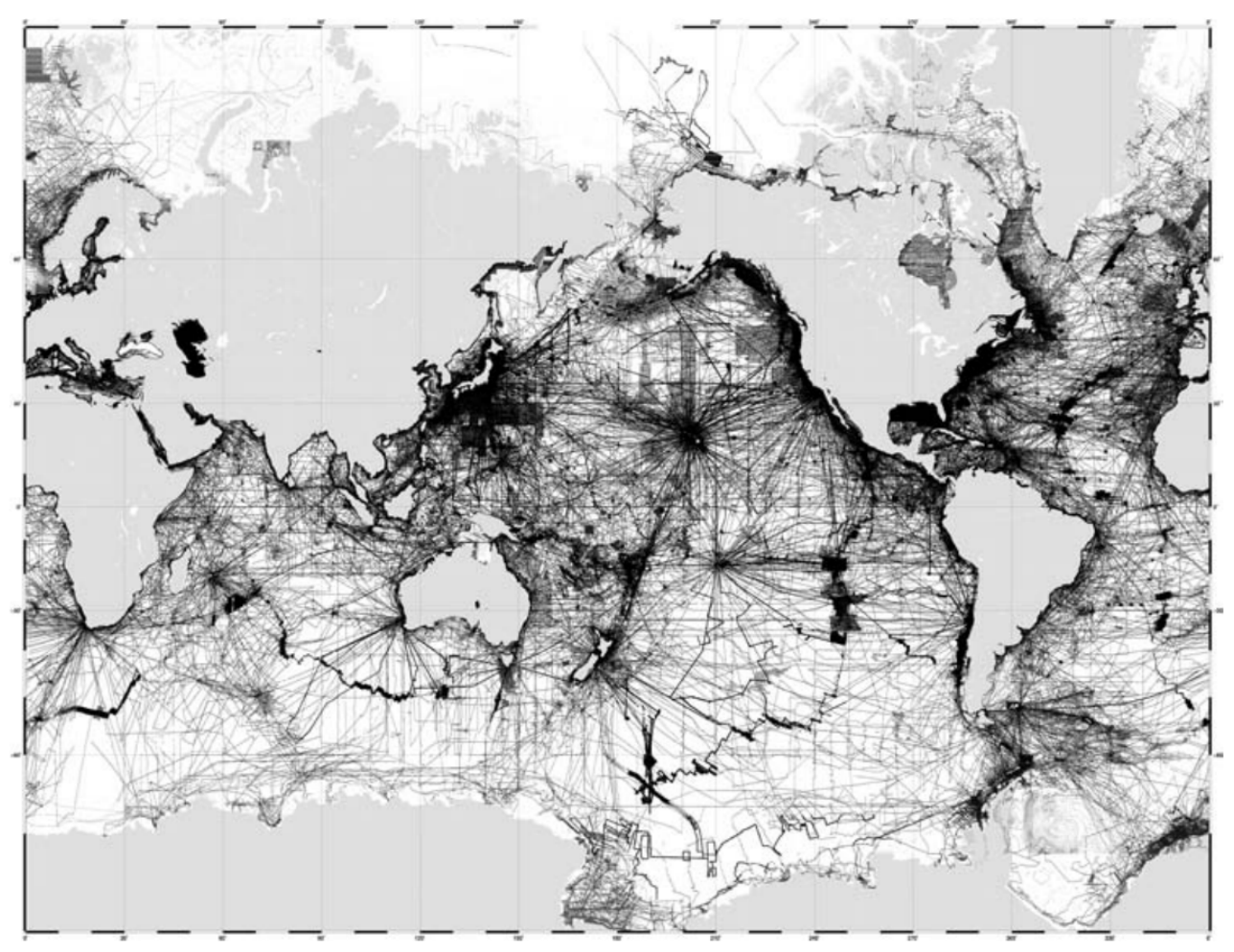

Figure 4.10: Here we see a map of the Earth, with the lines that were covered by echo-sounding ships shown in black. We can see how sparse those measurements are, especially in regions very relevant to tsunami forecasts, such as the Indian ocean. source: 69

gravitational deformation of the ocean surface by underwater structures, measured from space. 


\subsubsection{Satellite data}

The idea behind this kind of measurement is that the ideally spherical surface of the ocean is distorted by the presence of mountains or valleys in the ocean depth-profile, as is sketched in Fig 4.11. This effect is actually fairly weak, as a 2000-meter-high peak below sea level would only produce a 20-centimeters-high deviation from the spherical surface [68]. By measuring the deflection angle of a light beam by the surface of the ocean, we can find the surface deformation and reverse-engineer the shape and size of the underwater structure.

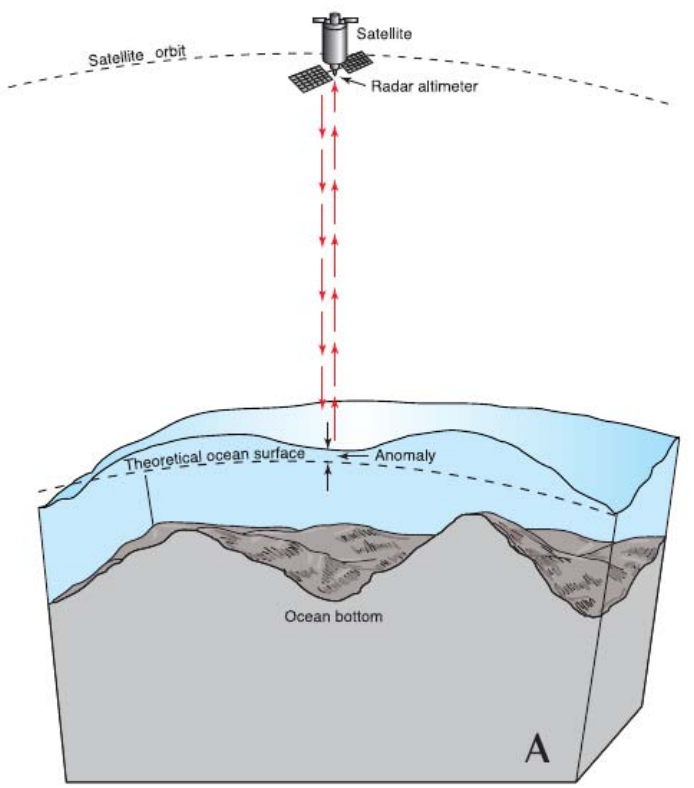

Figure 4.11: The presence of a bump in the bathymetry produces a variation in the surface gravity, leading to a slight deformation of the ocean surface. This deformation can be measured by a satellite which measures the deflection angle of a light beam sent to be reflected by the surface. From the surface deformation, an algorithm based on the theory of gravity can be used to reverse-engineer the size of the bump. source: 68

It is obvious that this method is subject to a lot of errors, first from the fact that the ocean surface is never at rest and therefore there is a lot of noise (which can, to some extent, be statistically removed). Furthermore, the process of deducing the shape and size of the underwater structure from its impact on the ocean surface is nonlinear and complicated, which can easily enhance small errors in the measurements.

Of course, we can use the more precise sonar measurements as a skeleton of the worldwide bathymetry map, then adjust the satellite-derived measurements and use interpolation to fill the holes in order to get a coherent bathymetry database. However, some problems can arise due to incompatible resolutions between data sets, as is discussed in [69]. Also, errors will tend to propagate in a intricate way, due to interpolation. Overall, the average absolute error in the database obtained by these combined methods is estimated between $100 \mathrm{~m}[69$ and $250 \mathrm{~m}$ [68]. Considering the average depth of the ocean $H_{0}=4 \mathrm{~km}$, we find a relative error approximately between $100 / 4000=2.5 \%$ and $250 / 4000=6.25 \%$. This is well within the range where branching is important.

Even though the noise enters the wave and ray equations as a multiplicative, thus nonlinear, term, we can assume that such errors can strongly change the wave intensity patterns. This is investigated in the next section. 


\subsubsection{Inacurrate forecasts due to uncertainties in the bathymetry database}

Now that we know that the bathymetry database is affected by some error that has an average size of somewhere between 100 and 250 meters, we want to see how much it will affect tsunami propagation and forecasts. In order to investigate this, we built a numerical experiment in the following way: we asked ourselves what would happen to the branching pattern if the bathymetry was slightly different, with variations from the original one that would be typically on the scale of 160 meters? The importance of the branching pattern is of course crucial, as it indicates the location of the highest, thus most destructive waves.

We considered the same bathymetry that was already studied in section 4.1 and added an error field of $4 \%$ standard deviation $(160 / 4000=4 \%)$, that we chose to be power-law correlated, because we do not know exactly how the error propagates through the interpolation process. In this way, we have a scale-free $4 \%$ error field. The original and modified bathymetries can be seen in Fig, 4.12. Now, we evolve two tsunamis with identical sources over these bathymetries and
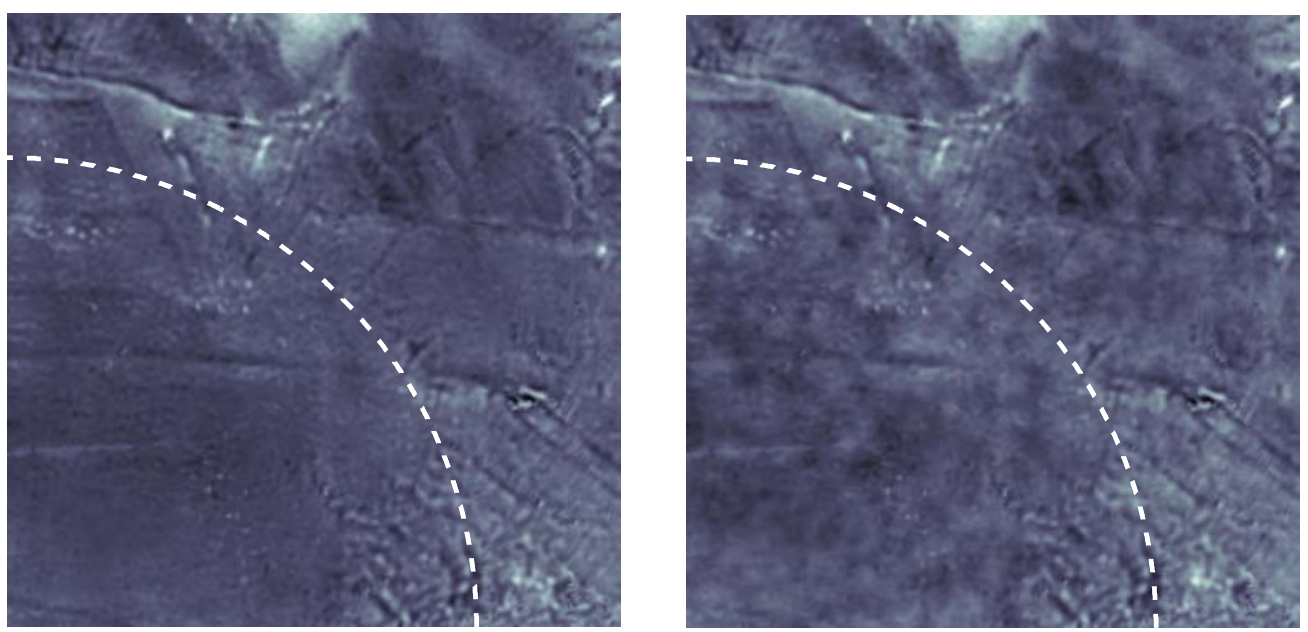

Figure 4.12: On the left panel is the original bathymetry, as taken from the GEBCO database and as we used it in section 4.1. On the right panel is the same bathymetry, but with an added power-law correlated error field of $4 \%$ standard deviation. They look very similar. The white dashed lines are related to Fig 4.13 and show where the cuts were made.

compare the integrated intensities, to see whether the outcome looks similar or not. We find that the branching patterns look very different, both in the location of the branches and their intensities. This result can be seen in Fig 4.13. Of course, we tested this procedure with different realizations and strength of the random error field and we also tested several exponents for the power-law. We found the effect to be robust against changes in most of the parameters. From this experiment, we can conclude that with an error of only a few percent in the bathymetry database, it is impossible to confidently predict the location of the strongest waves, thus strongly challenging our current capacity to accurately forecast tsunami disasters. Of course, point sources with a $20 \mathrm{~km}$ wavelength are rather idealized tsunami waves. However, our conclusions are not sensitive to the nature of the source, as can be seen in the next section.

\subsection{Alternative sources}

So far, the numerical simulations in real bathymetries we showed involved waves produced by idealized sources, i.e. localized point sources with a well-defined wavelength of $20 \mathrm{~km}$. This comparatively short wavelength was chosen because of the limited size of the island-free bathymetry region. There are, however, a couple of concerns that can be formulated with respect to this choice. The purpose of this section is to adress these concerns and to show that the effect we describe is robust against changes in the wavelength or in the geometry of the source. 

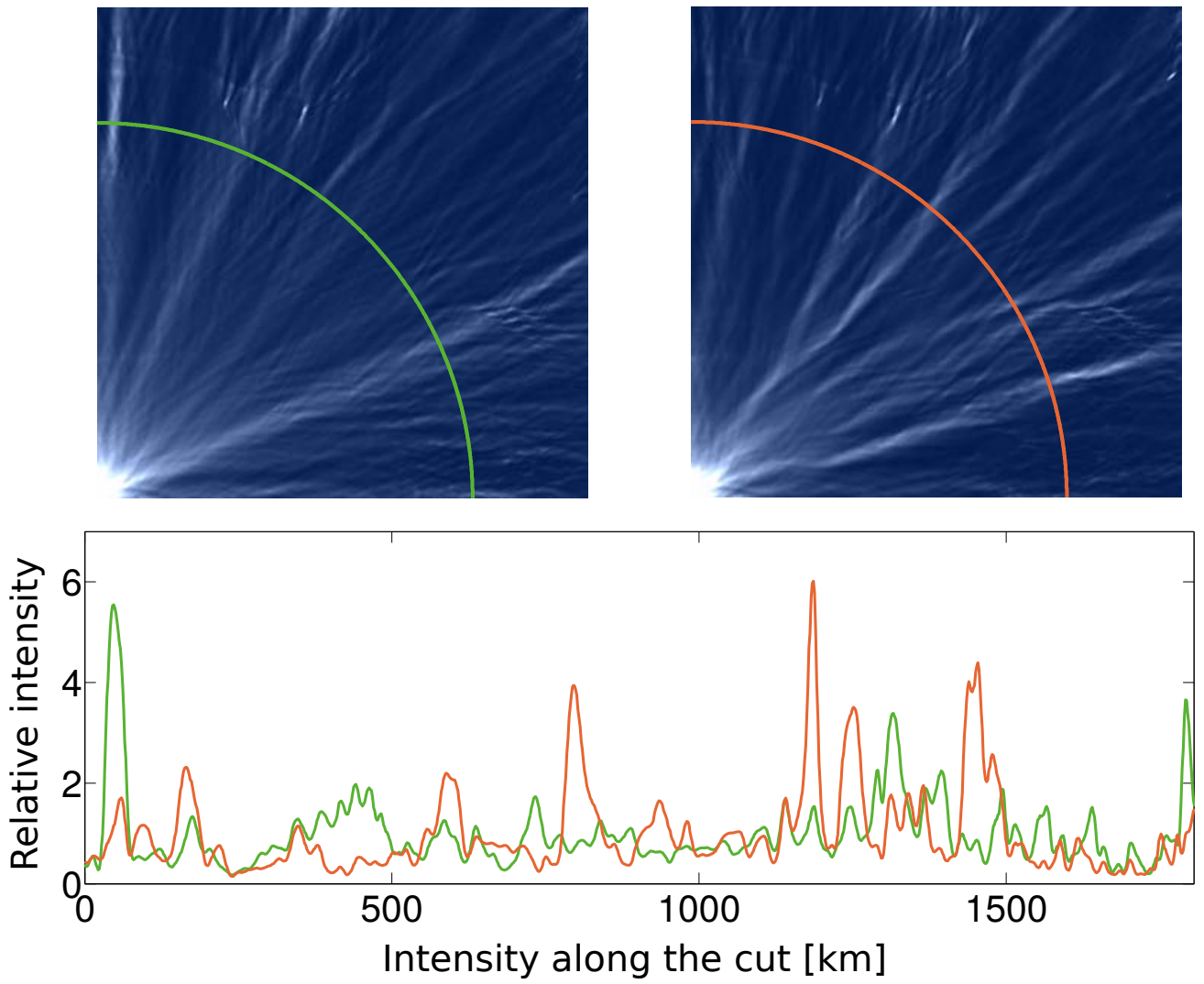

Figure 4.13: On the upper left panel is the intensity of the wave propagating over the original bathymetry, exactly as in Fig 4.3 . On the upper right panel is the intensity of the tsunami propagating over the modified bathymetry. We clearly see that the branching pattern is very different. Furthermore, the bottom panel shows the relative intensities along the green and orange cuts taken from the upper panels. Some branches, that were very strong in one case (up to 6 times the average height) simply disappear, whereas some new branches just come out of nowhere. The difference is striking, considering that the two floors that we used only differ by an average $4 \%$ error field.

\subsubsection{Larger wavelength}

The first thing that can be disputed here is our choice of wavelength of only $20 \mathrm{~km}$, which is at the very lower end of the tsunami window. Typical tsunamis of interest have wavelengths that are rather longer, of the order of $100 \mathrm{~km}$. We therefore show new simulations with a larger wavelength of $\lambda=100 \mathrm{~km}$ as well, even though the branching patterns are harder to visualize. However, cuts at different propagation distances reveal the same kind of strong fluctuations observed for short wavelengths. We present calculations on the exact same original floor (from Fig.4.1), along with two other simulations on slightly modified floors, in the same fashion as Fig 4.13. The result of these simulations can be seen in Fig 4.14 and shows that the phenomenon we describe is robust against changes in the wavelength.

\subsubsection{Fault-like source}

Another matter is the question of the geometry of the source. Indeed, real tsunamis are produced by earthquakes, which can have various geometries, such as a fault shape. As discussed at the beginning of this chapter, the study of realistic tsunami sources opens a wide field that goes beyond the scope of the present work. However, we decided to run some simulations using a dip-slip fault-like source, as described in appendix C, as an example. The results can be seen in Fig 4.15 and also show the expected branching patterns, confirming that the effect is robust against changes in shape. 

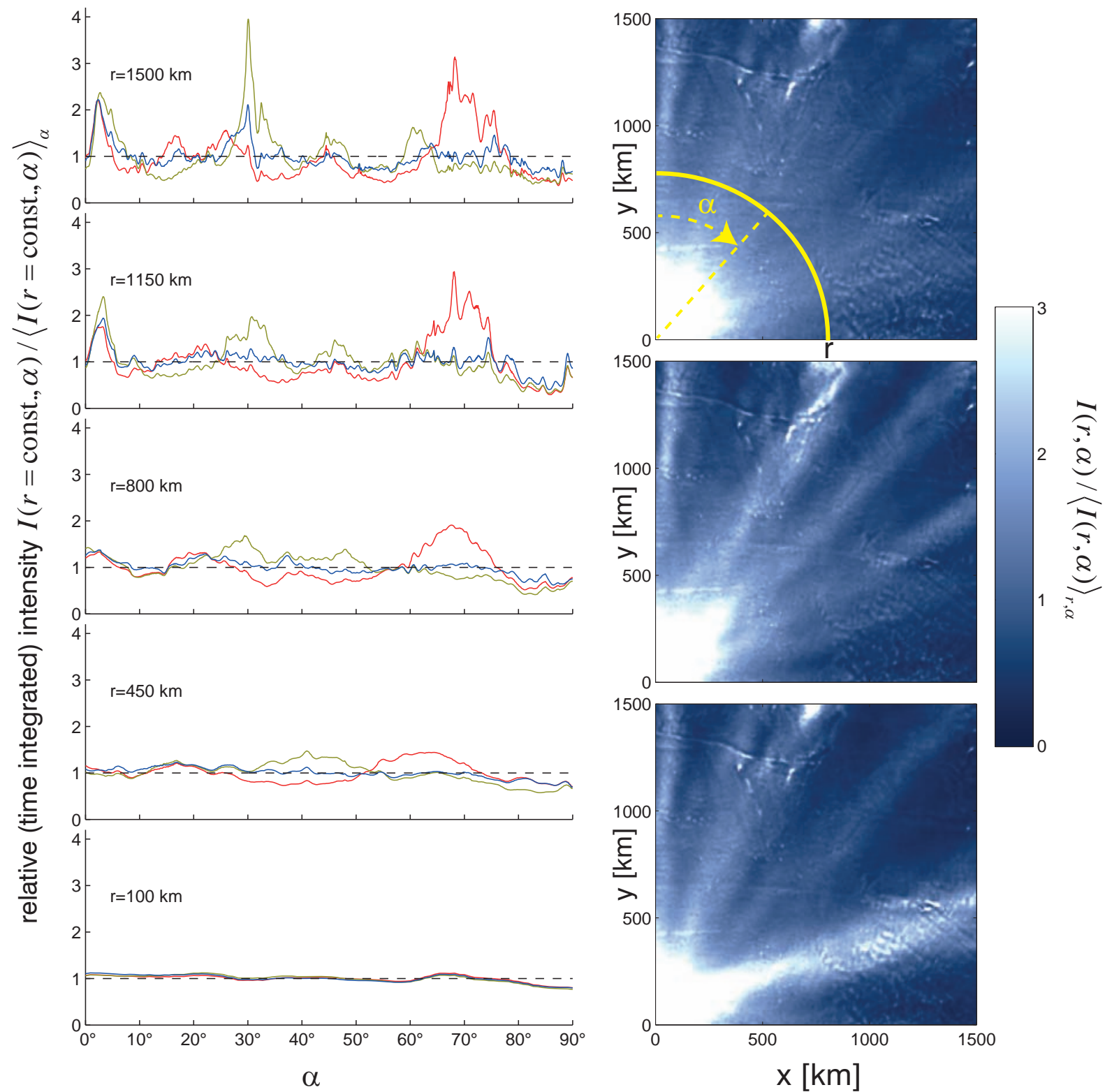

Figure 4.14: The three right panels show the integrated intensities of our three experiments, without errors (top) and with two different realizations of the noise (middle and bottom). We can clearly see important differences in the wave intensity patterns, both in strength and location. The left panels show some radial cuts in the intensities for the three experiments, at various distances from the source. The blue cuts represent the intensities on the original bathymetry. The green cuts correspond to the middle right panel and the red ones correspond to the bottom right panel. We can clearly see the large deviations described earlier. It is also interesting to notice that the intensities are very similar at the beginning (so, closer to the source) and progressively diverge from each other.

\subsection{Conclusion}

In this chapter, we showed the relevance of branched flow to the propagation of tsunami waves with a numerical experiment using real ocean floor data. Developing the theory of branched flow and first caustics statistics in the case of shallow water rays, we were able to estimate the mean distance from the source to the first caustics in terms of the fundamental parameters of the bathymetry, namely its standard deviation $\epsilon$ and its correlation length $l_{c}$. We confirmed this 

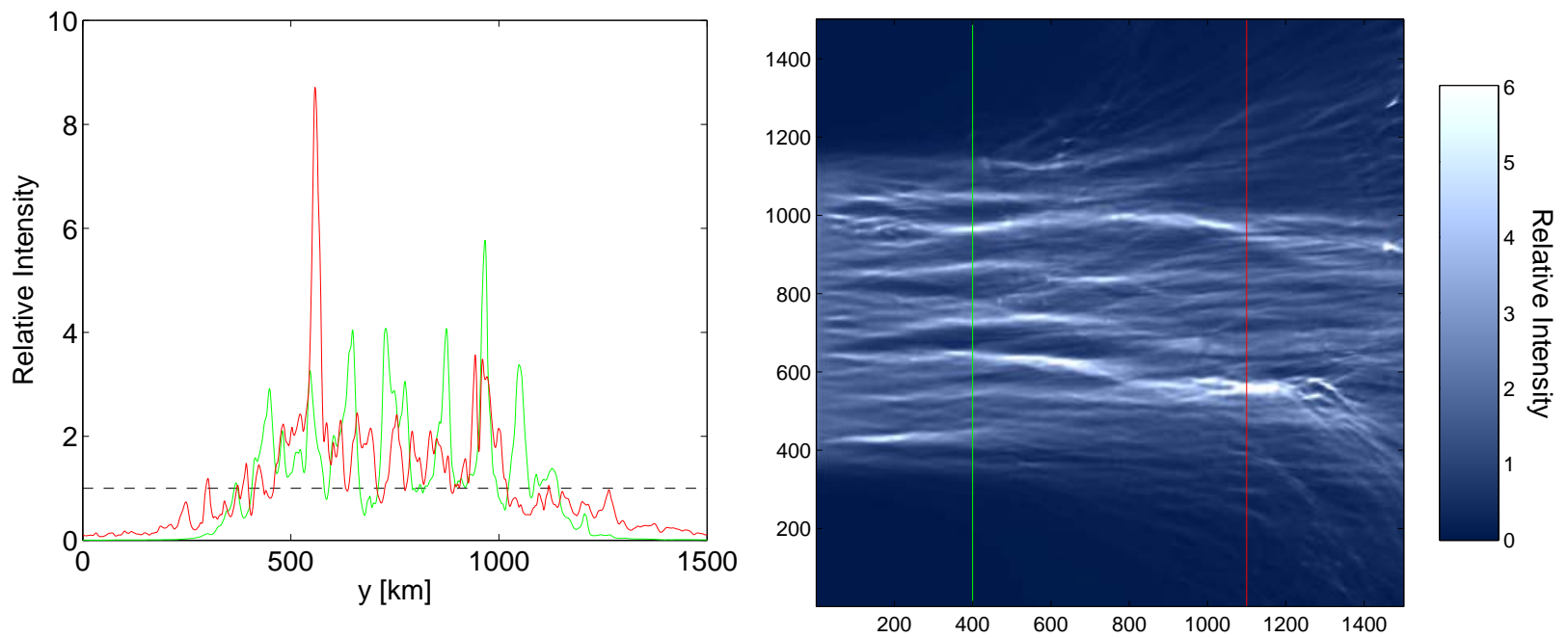

Figure 4.15: The right panel shows the integrated intensities for a fault-like source. We clearly see the branching patterns, which can also be seen in the cuts on the left panel. The relative intensities are given with respect to the mean along the cuts.

scaling law with extensive numerical simulations, which helped us to determine the missing prefactor in our analytical results. From our scaling analysis, we realized that small fluctuations in the ocean floor topography, typically within the error margin with which the bathymetry is known, are sufficient to completely change the branching patterns, therefore making it very difficult to predict the location of the most dangerous waves in case of a tsunami disaster. This effect is robust against changes in the source parameters.

Our theoretical analysis so far describes a very ideal case of a tsunami evolving over an isotropic bathymetry with a single correlation length. Real bathymetries, however, exhibit clear anisotropies, e.g. underwater ridges, and tend to be correlated on many-scales, as the geological processes responsible for their structure are many-fold. In the next chapter, we will therefore extend our theory to the case of such random fields. We will deal with anisotropic potentials, both for classical rays and shallow water waves, and also multi-length-scale media, once again for both systems. These new theories will of course be supported by extensive numerical simulations. 



\section{Chapter 5}

\section{Branched flow in media with multiple correlation lengths}

In the previous chapters of this thesis, we developed the stochastic theory of branching of tsunami rays and shallow water waves in the simplest possible random bathymetries, i.e. isotropic random fields correlated on a single length scale. Even though this model already allowed us to draw interesting conclusions on the impact of branched flow on tsunami waves, it is clear that most real systems will exhibit some anisotropies, e.g. ridges in the ocean floor topography, semiconductors with anisotropic effective mass or anisotropic turbulent air flows. Furthermore, most real systems exhibit correlations on several length scales, e.g. ocean floors are affected by various geological process occurring on different scales. We are therefore also interested in the statistics of random caustics in media that are correlated on several length scales. In this chapter, we want to quantify the effect of anisotropy and multi-length-scale correlations on the distance to the first caustics. Even though those two properties are likely to be both present, we will study them separately, as it will make the analysis more clear and the interplay between them is straightforward.

\subsection{Global anisotropy}

We want to model systems that are evolving in globally anisotropic random media, which we will assume to be homogeneous. As all the statistical properties of the random potential we are modeling lie in the correlation function (see appendix A), it seems appropriate to model a global anisotropy in its definition. In two dimensions, the transformation giving rise to global anisotropies is a combination of rotations and stretching in correlation space, which can be written as the consecutive effects of a rotation matrix $\mathcal{T}_{R}$ and a stretching matrix $\mathcal{T}_{S}$ acting on the space variable $\vec{x}$ in the correlation function $c(\vec{x})$

$$
\vec{x} \longrightarrow \vec{x}^{\prime}=\mathcal{T} \vec{x} ; \mathcal{T}=\mathcal{T}_{S} \mathcal{T}_{R},
$$

with $\mathcal{T}_{R}$ and $\mathcal{T}_{S}$ respectively defined by the one- and two-parameter matrices

$$
\mathcal{T}_{R}(\alpha)=\left(\begin{array}{cc}
\cos \alpha & \sin \alpha \\
-\sin \alpha & \cos \alpha
\end{array}\right) \text { and } \mathcal{T}_{S}(a, b)=\left(\begin{array}{cc}
a & 0 \\
0 & b
\end{array}\right)
$$

Even though we will be considering transformations of the form described in Eq. 5.1 5.2), we won't restrict ourselves to this specific class of transformations in the derivations and we will consider a linear transformation $\mathcal{T}$ that can be very general up to some constraints described below. We start by considering a transformation from $\vec{x} \longrightarrow \vec{x}^{\prime}=\mathcal{T} \vec{x}$. In previous chapters, we restricted ourselves to the general case of correlation functions $c(\vec{x})=\epsilon^{2} f\left(\vec{x} \cdot \vec{x} / l_{c}\right)$, which depended only on the scalar product and a single correlation length $l_{c}$. In our new system, however, the scalar product will be changed by the transformation $\mathcal{T}$ and we decide to absorb the 
correlation length in the definition of $\mathcal{T}_{S}$, as it is not unique anymore. Considering this, we have $c\left(\vec{x}^{\prime}\right)=\epsilon^{2} f\left(\vec{x}^{\prime} \cdot \vec{x}^{\prime}\right)=\epsilon^{2} f((\mathcal{T} \vec{x}) \cdot(\mathcal{T} \vec{x}))$, where the scalar product now yields

$$
\vec{x}^{\prime} \cdot \vec{x}^{\prime}=\vec{x}^{T} \mathcal{T}^{T} \mathcal{T} \vec{x}=\mathcal{M}_{11} x^{2}+2 \mathcal{M}_{a} x y+\mathcal{M}_{22} y^{2},
$$

with the $\mathcal{M}_{i j}$ being the elements of $\mathcal{M}=\mathcal{T}^{T} \mathcal{T}$ with

$$
\mathcal{M}_{a}=\frac{1}{2}\left(\mathcal{M}_{12}+\mathcal{M}_{21}\right) .
$$

The $\mathcal{M}_{i j}$ can be very general in what follows, as long as $\mathcal{M}_{11} \neq 0$. The case $\mathcal{M}_{11}=0$ does not make much sense anyway considering that, for instance, it implies $a=b=0$ in the case of Eqs. 5.1 5.2), which means that the correlation function is flat, i.e. the random potential is flat. The function $f: \mathbb{R} \rightarrow \mathbb{R}$, that we will require to be up to four times differentiable for some cases presented below, needs to have the following property

$$
\int_{-\infty}^{+\infty} \mathrm{d} z f^{(n)}\left(z^{2}\right)=: \kappa_{n}<\infty
$$

for $n=\{0,1,2\}$, as can already be understood from section 4.3. The function $f(\vec{x} \cdot \vec{x})$ is now the correlation function (up to a factor of $\epsilon^{2}$ ) with $f(0)=1$. Here, we need to realize that the only differences between the isotropic and the anisotropic case lie in the definition of the correlation function and the fact that the explicit choice of the propagation direction now matters. Indeed, the procedure shown in chapters 2 and 4 makes use of the random potential when going in the quasi-2D approximation, transforming it into a white noise term with strength $\sigma_{m}$, defined by

$$
\sigma_{m}^{2}=\left.\frac{(-1)^{m}}{2} \int_{-\infty}^{+\infty} \mathrm{d} x\left[\frac{\partial^{2 m} c(x, y)}{\partial y^{2 m}}\right]\right|_{y=0},
$$

where we had defined $x$ as the propagation direction and $y$ as the transverse direction. This choice was absolutely straightforward in the isotropic case, as the correlation function would be the same in every choice of axis, as the scalar product is invariant under rotations. Now, we have an extra term in the correlation function, coming from the matrix $\mathcal{M}$, which will be different in each choice of axis except for very few special cases. We will maintain that $x$ is the propagation direction and $y$ is the transverse direction, but then we will need to adapt the form of the matrix $\mathcal{M}$ to these new axis, depending on their orientation with respect to the anisotropy. The angle $\alpha$ in Eq. (5.2) describes this orientation, by defining what is the angle of the propagation direction for the ray under consideration, in our specific model (which also explains why we decided to describe the transformation in this fashion). We have

$$
c(x, y)=\epsilon^{2} f\left(\vec{x}^{\prime} \cdot \vec{x}^{\prime}\right)=\epsilon^{2} f\left(\mathcal{M}_{11} x^{2}+2 \mathcal{M}_{a} x y+\mathcal{M}_{22} y^{2}\right) \equiv \epsilon^{2} f(R)
$$

where we defined $R=\mathcal{M}_{11} x^{2}+2 \mathcal{M}_{a} x y+\mathcal{M}_{22} y^{2}$ to make everything shorter afterwards. The impact of the transformation in the form of Eqs.5.1 5.2 on the correlation function and the random potential can be seen in Fig 5.1 .

We now have a well-defined way to write the correlation function in terms of the angle defined by the propagation direction, which means that the integrals in Eq.55.6) for the $\sigma_{i}$ are well defined and now depend on the couple of stretching factors $(a, b)$ and the propagation orientation $\alpha$, instead of simply $l_{c}$. Of course, we could also use any other kind of transformation, the matrix of which would then need to be changed into the base of the new coordinate system of appropriate propagation/transverse direction. We will do most of the derivations below considering a transformation $\mathcal{M}$ as general as possible but in the right basis according to the orientation with respect to the direction of the ray under consideration. Only in the end will we make use of the definitions in Eq. 5.1 5.2). This represents the main change from the isotropic case, but there will be an extra difference in the shallow water case, as will be shown in section 5.1.2. 

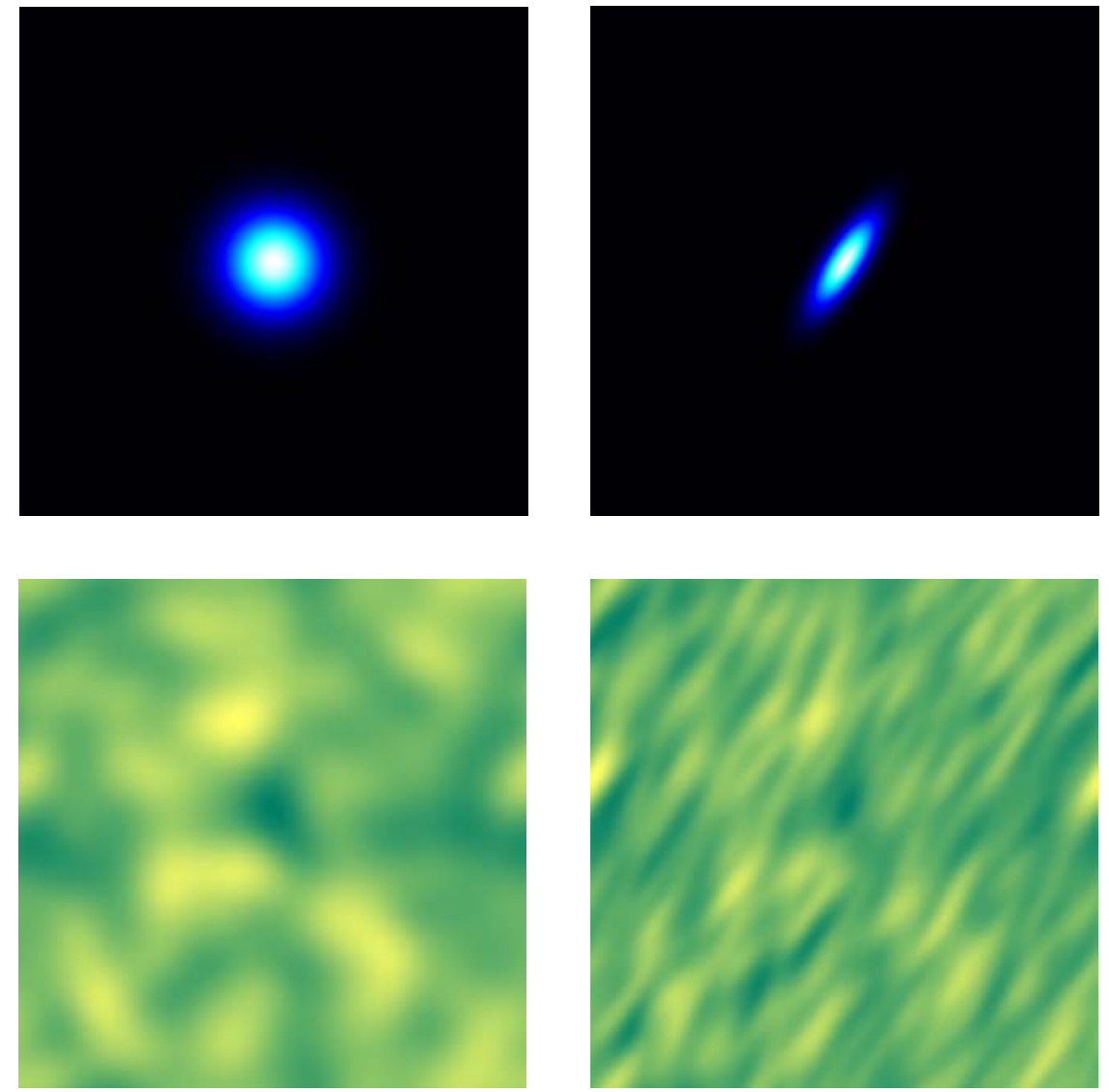

Figure 5.1: The upper left panel shows an isotropic correlation function, which spans the random potential shown in the lower left panel. The upper right panel shows the same correlation function, after we applied a transformation of the type Eq.5.1.5.2), with the values $a=1, b=3$ and $\alpha=1$. The random potential that spans from this correlation function can be seen in the bottom right panel. We can see how this transformation leads to a strong anisotropy in the potential, which takes the chosen orientation defined by $\alpha$.

\subsubsection{Classical rays}

From section 2.6, we know that we compute the distance to the first caustics by looking at the curvature equation and by finding the mean first passage time through the value $u=-\infty$, starting from some initial value $u_{0}$ that typically takes the value $u_{0}=0$ for plane waves and $u_{0}=+\infty$ for point sources. In quasi- $2 \mathrm{~d}$, the curvature equation became the Langevin equation given by

$$
\frac{d}{d t} u(t)+u^{2}(t)+\sigma_{2} \Gamma(t)=0,
$$

where we immediately notice that the only difference with the isotropic case will be the value of $\sigma_{2}$. This means that we can reuse the result from section 2.6 , simply with a different $\sigma_{2}$ that is given by

$$
\sigma_{2}^{2}=\left.\frac{1}{2} \int_{-\infty}^{+\infty} \mathrm{d} x\left[\frac{\partial^{4} c(x, y)}{\partial y^{4}}\right]\right|_{y=0}=\left.\frac{\epsilon^{2}}{2} \int_{-\infty}^{+\infty} \mathrm{d} x\left[\frac{\partial^{4}}{\partial y^{4}} f(R(x, y))\right]\right|_{y=0}
$$

The derivatives are straightforward and yield 


$$
\frac{\partial^{4}}{\partial y^{4}} f(R)=f^{(4)}(R)\left[2 \mathcal{M}_{a} x+2 \mathcal{M}_{22} y\right]^{4}+12 \mathcal{M}_{22} f^{(3)}(R)\left[2 \mathcal{M}_{a} x+2 \mathcal{M}_{22} y\right]^{2}+12 \mathcal{M}_{22}^{2} f^{(2)}(R)
$$

where once again, $f^{(n)}(R)=\frac{d^{n}}{d R^{n}} f(R)$. Inserting this into the integral for $\sigma_{2}$ and using the fact that $\left.R\right|_{y=0}=\mathcal{M}_{11} x^{2}$ gives

$$
\sigma_{2}^{2}=\frac{\epsilon^{2}}{2} \int_{-\infty}^{+\infty} \mathrm{d} x\left[16 \mathcal{M}_{a}^{4} x^{4} f^{(4)}\left(\mathcal{M}_{11} x^{2}\right)+48 \mathcal{M}_{a}^{2} \mathcal{M}_{22} x^{2} f^{(3)}\left(\mathcal{M}_{11} x^{2}\right)+12 \mathcal{M}_{22}^{2} f^{(2)}\left(\mathcal{M}_{11} x^{2}\right)\right] .
$$

This contains three types of integrals, which in the end will all produce the same integral. Indeed, the first term can be integrated by parts

$$
\int_{-\infty}^{+\infty} \mathrm{d} x x^{4} f^{(4)}\left(\mathcal{M}_{11} x^{2}\right)=\left.\left[x^{3} \frac{1}{2 \mathcal{M}_{11}} f^{(3)}\left(\mathcal{M}_{11} x^{2}\right)\right]\right|_{-\infty} ^{+\infty}-\frac{3}{2 \mathcal{M}_{11}} \int_{-\infty}^{+\infty} \mathrm{d} x x^{2} f^{(3)}\left(\mathcal{M}_{11} x^{2}\right) .
$$

The boundary term vanishes (odd function) and therefore we are left with an integral of the same type as in the second term of Eq.(5.11), which can also be integrated by parts

$$
\int_{-\infty}^{+\infty} \mathrm{d} x x^{2} f^{(3)}\left(\mathcal{M}_{11} x^{2}\right)=\left.\left[x \frac{1}{2 \mathcal{M}_{11}} f^{(2)}\left(\mathcal{M}_{11} x^{2}\right)\right]\right|_{-\infty} ^{+\infty}-\frac{1}{2 \mathcal{M}_{11}} \int_{-\infty}^{+\infty} \mathrm{d} x f^{(2)}\left(\mathcal{M}_{11} x^{2}\right) .
$$

Once again, the boundary term vanishes and we are left with the same integral as the last term of Eq. (5.11), which yields

$$
\int_{-\infty}^{+\infty} \mathrm{d} x f^{(2)}\left(\mathcal{M}_{11} x^{2}\right)=\frac{1}{\sqrt{\mathcal{M}_{11}}} \int_{-\infty}^{+\infty} \mathrm{d} z f^{(2)}\left(z^{2}\right)=\frac{\kappa_{2}}{\sqrt{\mathcal{M}_{11}}}
$$

where $\kappa_{2}$ is the constant defined Eq. (5.5), which is independent of the parameters of the transformation, and we used the change of variable $z=\sqrt{\mathcal{M}_{11}} x$. These calculations explain why we needed $f(R)$ to be four times differentiable and $\mathcal{M}_{11} \neq 0$.

We therefore showed that, as long as the correlation function has the above-mentioned properties, we find

$$
\sigma_{2}^{2}=\frac{6 \kappa_{2} \epsilon^{2}}{\mathcal{M}_{11}^{5 / 2}}\left[\mathcal{M}_{a}^{4}-2 \mathcal{M}_{a}^{2} \mathcal{M}_{22} \mathcal{M}_{11}+\mathcal{M}_{11}^{2} \mathcal{M}_{22}^{2}\right]
$$

Going back to our well-defined case of rotations and stretching from Eq. (5.1) 5.2), in which the matrix elements $\mathcal{M}_{i j}$ are defined as

$$
\begin{aligned}
& \mathcal{M}_{11}=a^{2} \cos ^{2} \alpha+b^{2} \sin ^{2} \alpha \\
& \mathcal{M}_{12}=\mathcal{M}_{21}=\mathcal{M}_{a}=\left(a^{2}-b^{2}\right) \sin \alpha \cos \alpha \\
& \mathcal{M}_{22}=a^{2} \sin ^{2} \alpha+b^{2} \cos ^{2} \alpha
\end{aligned}
$$

we find, after some straightforward trigonometric manipulations,

$$
\sigma_{2}^{2}=\frac{6 \kappa_{2} \epsilon^{2} a^{4} b^{4}}{\mathcal{M}_{11}^{5 / 2}}=\frac{6 \kappa_{2} \epsilon^{2} a^{4} b^{4}}{\left(a^{2} \cos ^{2} \alpha+b^{2} \sin ^{2} \alpha\right)^{5 / 2}} .
$$

Using the result for the time to the first caustics found in section 2.6 (Eq. 2.46 ) and the new $\sigma_{2}$, we find 


$$
\left\langle t_{f}\right\rangle=C_{s}^{c}\left(6 \kappa_{2}\right)^{-1 / 3} \epsilon^{-2 / 3} a^{-4 / 3} b^{-4 / 3}\left(a^{2} \cos ^{2} \alpha+b^{2} \sin ^{2} \alpha\right)^{5 / 6},
$$

where $C_{s}^{c}$ is the constant for the classical ray case and depends on which initial condition $s=\{p w, p s\}$ we use. We have $C_{p w}^{c}=3.31$ for plane waves and $C_{p s}^{c}=4.97$ for point sources [23]. We note that, if we go back to the isotropic case $a=b=l_{c}^{-1}$, we exactly recover the result from chapter 2 .

In order to verify this result numerically, we fixed the value of $a=$ const, which basically defines a scale for the whole system. Then, we did simulations with various values for $b$ and $\alpha$ over 400 different potentials. The results are plotted in figure Fig 5.2 and Fig 5.3 . We can see an excellent agreement with the theoretical result derived above. We can see in Fig.5.3 that the angular

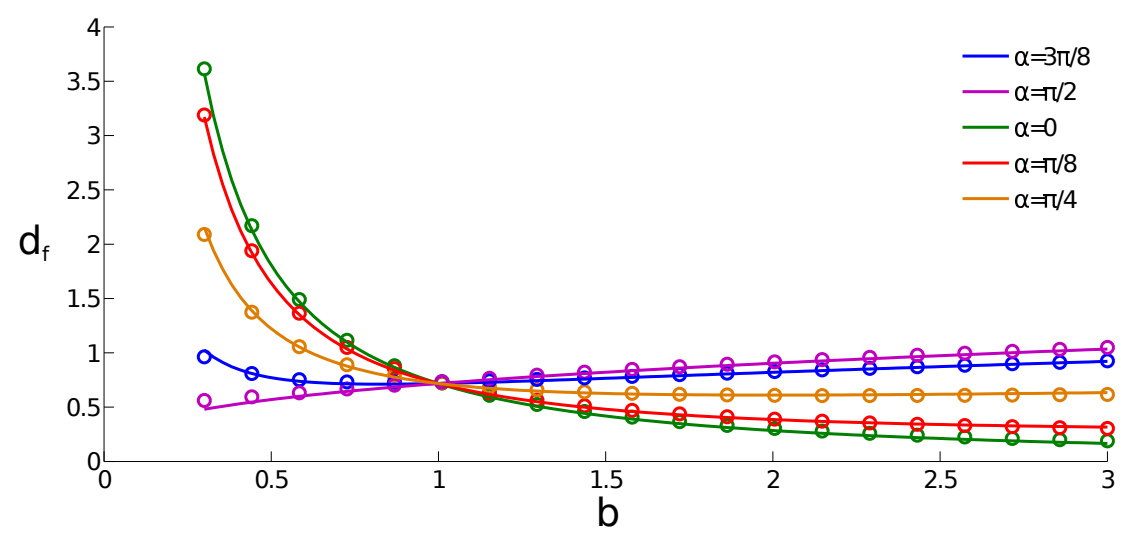

Figure 5.2: These curves show the analytical results for the distance to the first caustics $d_{f}$ as functions of $b$, as predicted by Eq.(5.18). The dots are the average values we obtained from numerical simulations. Each color corresponds to one angle $\alpha$, as can be seen in the legend. These results were obtained with plane wave initial conditions. The units for the distance are arbitrary.

distribution of the distance to the first caustics in anisotropic potentials exhibits a characteristic peanut-like shape.

\subsubsection{Shallow water waves}

Since we showed in section 4.2 that the method using the curvature equation does not work for water waves, we will have to do the computation from the ray equations. As in the previous section, we start from the associated Langevin equations obtained in section 4.3

$$
\begin{aligned}
& \dot{y}=\left(1-\sigma_{0} \Gamma_{1}(t)\right) p, \\
& \dot{p}=\frac{c_{0}^{2} \sigma_{1} \Gamma_{2}(t)}{2} .
\end{aligned}
$$

This means that we have to compute $\sigma_{0,1}$ from Eq.(5.6), which gives rise to two integrals

$$
\begin{aligned}
\sigma_{0}^{2} & =\left.\frac{\epsilon^{2}}{2} \int_{-\infty}^{+\infty} \mathrm{d} x f(R(x, y))\right|_{y=0}=\frac{\epsilon^{2}}{2} \int_{-\infty}^{+\infty} \mathrm{d} x f\left(\mathcal{M}_{11} x^{2}\right)=\frac{\epsilon^{2}}{2 \mathcal{M}_{11}^{1 / 2}} \kappa_{0} \\
\sigma_{1}^{2} & =-\left.\frac{\epsilon^{2}}{2} \int_{-\infty}^{+\infty} \mathrm{d} x \partial_{y}^{2} f(R(x, y))\right|_{y=0}=-\epsilon^{2} \int_{-\infty}^{+\infty} \mathrm{d} x\left[2 f^{(2)}(R)\left[\mathcal{M}_{a} x+\mathcal{M}_{22} y\right]^{2}+\mathcal{M}_{22} f^{(1)}(R)\right](5,22)
\end{aligned}
$$




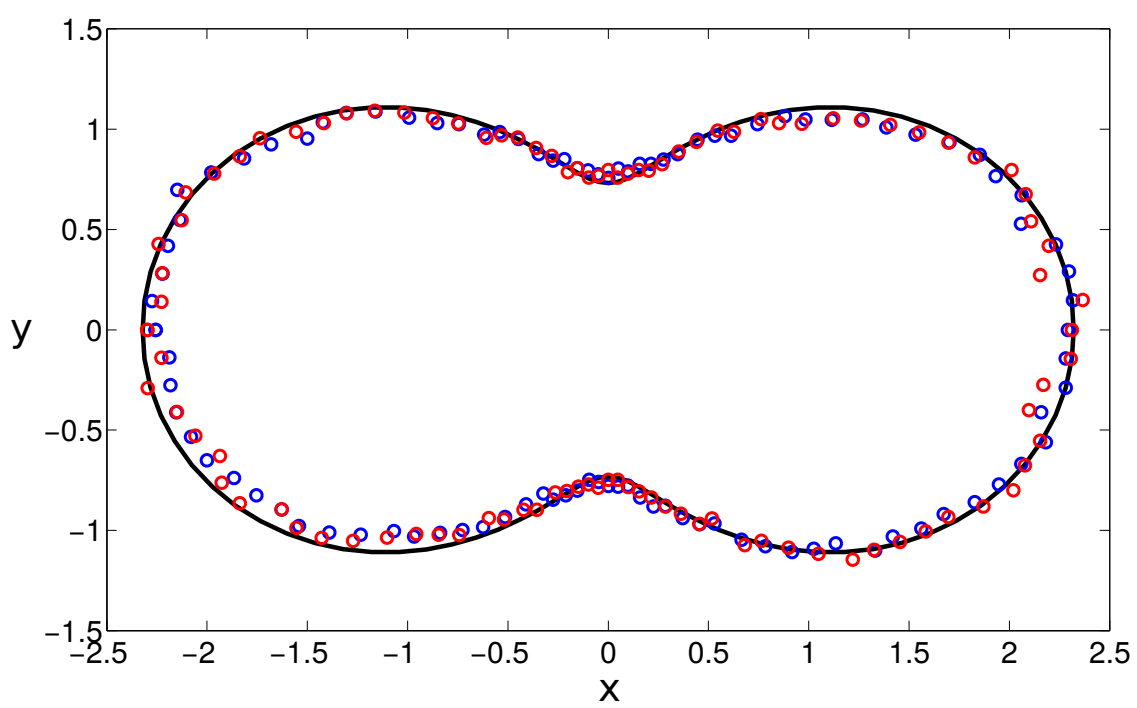

Figure 5.3: The black curve shows the analytical result for the distance to the first caustics as a function of $\alpha$ with a fixed $b=0.5$, as predicted by Eq. (5.18). The blue dots are the average distance to the first caustics we obtained from numerical simulations by considering the initial angle of each ray. The red dots show the same quantity, except that we sorted the data point by the average angle of the whole trajectory, from its start to the caustic. We see that both measures give excellent agreement. These results were obtained from point source initial conditions and we used bins to discretize the angles. The units for the distance are arbitrary.

the second of which needs similar treatment to that in section 5.1.1, using

$$
\int_{-\infty}^{+\infty} \mathrm{d} x x^{2} f^{(2)}\left(\mathcal{M}_{11} x^{2}\right)=\left[x \frac{1}{2 \mathcal{M}_{11}} f^{(1)}\left(\mathcal{M}_{11} x^{2}\right)\right]_{-\infty}^{+\infty}-\frac{1}{2 \mathcal{M}_{11}} \int_{-\infty}^{+\infty} \mathrm{d} x f^{(1)}\left(\mathcal{M}_{11} x^{2}\right) .
$$

Since the boundary term vanishes, all the integrals in Eq. 5.22 are of the same order and we find

$$
\sigma_{1}^{2}=\frac{\epsilon^{2} \kappa_{1}}{\mathcal{M}_{11}^{3 / 2}}\left[\mathcal{M}_{a}^{2}-\mathcal{M}_{11} \mathcal{M}_{22}\right]
$$

Using the definition of the $\mathcal{M}_{i j}$ in Eq.(5.16), along with a little trigonometry, we find

$$
\sigma_{1}^{2}=\frac{\epsilon^{2} \kappa_{1} a^{2} b^{2}}{\mathcal{M}_{11}^{3 / 2}}
$$

We now have the two $\sigma_{i}$ that were necessary for the computation in section 4.3. Our previous derivation from section 4.3 went as follows: we considered that, at the time of the first caustics $t_{f}$, the typical deviation of the ray in the transverse direction, which can be estimated with $\left\langle y^{2}(t)\right\rangle$, should scale with the correlation length $l_{c}$

$$
\left\langle y^{2}\left(t_{f}\right)\right\rangle \sim l_{c}^{2} .
$$

Solving for $\left\langle y^{2}(t)\right\rangle$ follows the exact same computation, using the two new $\sigma_{0,1}$ that we just computed. Our problem comes from the fact that there is no unique correlation length $l_{c}$ anymore. Instead, we have to use an effective correlation length that will depend on the angle $\alpha$ and the stretching factors $a$ and $b$, as it will depend on the choice of the transverse direction. In order to understand how these parameters will impact this effective correlation length, let us consider the simplest case where $\alpha=0$, then $\mathcal{M}=\operatorname{diag}\left(a^{2}, b^{2}\right)$. In this case, the correlation 
function would be $c(x, y)=\epsilon^{2} f\left(a^{2} x^{2}+b^{2} y^{2}\right)$, which, when compared to the isotropic case of $c(x, y)=\epsilon^{2} f\left(x^{2} / l_{c}^{2}+y^{2} / l_{c}^{2}\right)$, means that these two stretching factors are to be understood as inverse correlation lengths, one for each direction. When $a \neq b$ and for any angle $\alpha$, the value of this inverse correlation length will be given by the radius $r_{a b}(\alpha)$ of an ellipse with axes $a$ and $b$

$$
r_{a b}(\alpha)=\frac{a b}{\left(a^{2} \sin ^{2} \alpha+b^{2} \cos ^{2} \alpha\right)^{1 / 2}},
$$

This expression gives us the radius of the ellipse for the angle $\alpha$, which is the angle describing the propagation direction. This means that, if we want to have the length in the transverse direction, we need to add $\pi / 2$ to the angle, which simply exchanges the sine and the cosine in the formula, such that we have

$$
r_{a b}^{\perp}(\alpha)=\frac{a b}{\left(a^{2} \cos ^{2} \alpha+b^{2} \sin ^{2} \alpha\right)^{1 / 2}},
$$

where $r_{a b}^{\perp}(\alpha)$ is now the radius of the ellipse in the transverse direction. Fig 5.4 illustrates the procedure we just described. We note that its denominator is none other than $\mathcal{M}_{11}$. As $r_{a b}^{\perp}(\alpha)$ represents an inverse correlation length, we can deduce that the effective correlation length in the transverse direction $l_{e}$ is given by

$$
l_{e}=\frac{1}{r_{a b}^{\perp}(\alpha)} .
$$

Then, considering our previous argument

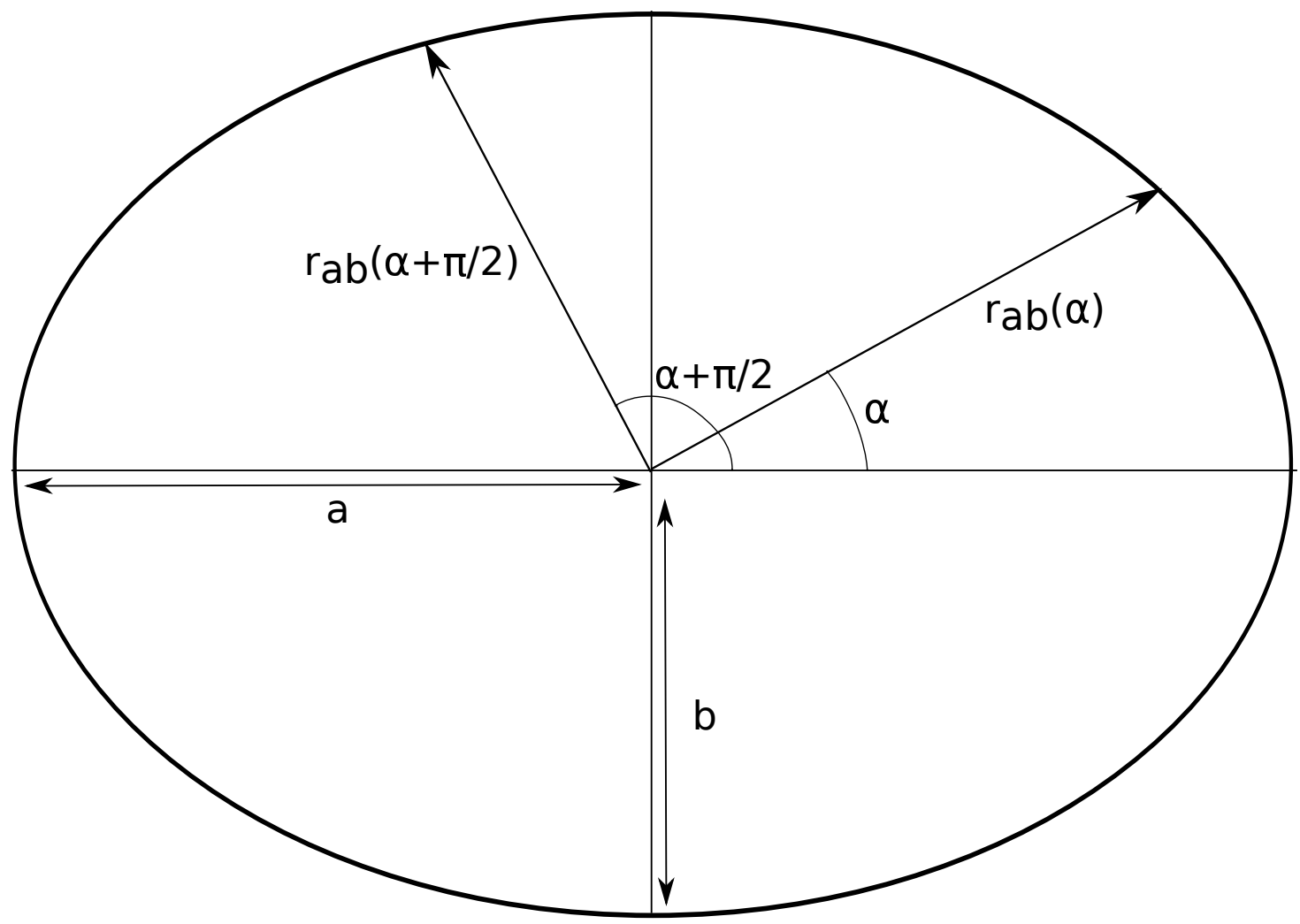

Figure 5.4: The ellipse described by the two main axis $a$ and $b$ in correlation space. The distances in this space are to be thought of as inverse correlation lengths.

$$
\left\langle y^{2}\left(t_{f}\right)\right\rangle \sim l_{e}^{2}=\left[\frac{1}{r_{a b}^{\perp}(\alpha)}\right]^{2}
$$

and using the result from section 4.3 for $\left\langle y^{2}(t)\right\rangle$ with the small $\sigma_{0}$ approximation (see Eq.4.58) 


$$
\left\langle y^{2}(t)\right\rangle \approx \frac{1}{6} c_{0}^{4} \sigma_{1}^{2} t^{3}
$$

we find

$$
\left\langle t_{f}\right\rangle=-C_{s}^{w} c_{0}^{-1} \epsilon^{-2 / 3}\left(\frac{6}{\kappa_{1}}\right)^{1 / 3} a^{-4 / 3} b^{-4 / 3}\left(a^{2} \cos ^{2} \alpha+b^{2} \sin ^{2} \alpha\right)^{5 / 6},
$$

where $C_{s}^{w}$ is the constant for the water ray case and depends on which initial condition $s=\{p w, p s\}$ we use. We have $C_{p w}^{c} \simeq 2.55$ for plane waves and $C_{p s}^{c} \simeq 3.78$ for point sources, as was obtained numerically in section 4.4. We note once again that, if we go back to the isotropic case $a=b=l_{c}^{-1}$, we recover the exact result from chapter 4 . We also note that, up to the prefactor, the dependency on the different parameters $\epsilon, a, b$ and $\alpha$ is exactly the same as for classical rays. As in the previous section, we will numerically verify the scaling in $b$ and $\alpha$ by setting $a=$ const in the simulations for rays. The results can be seen in Fig 5.5 and Fig 5.6.

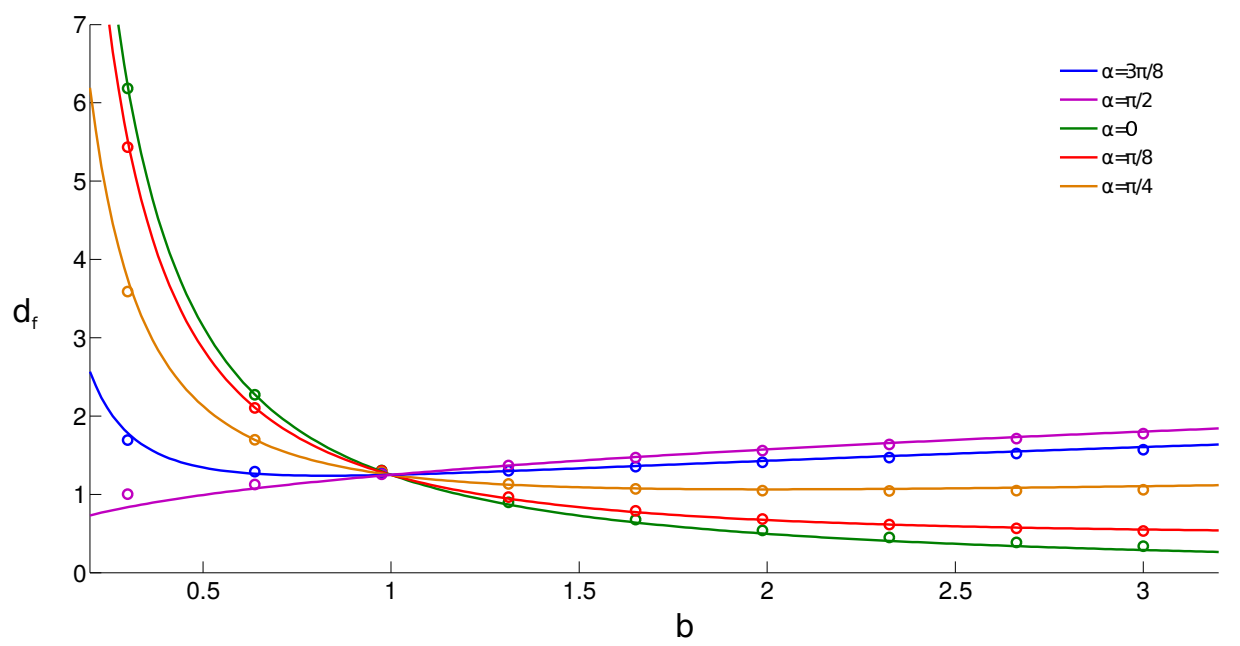

Figure 5.5: Here we have scaling curves for five different angles (see legend), plotting the distance to the first caustics $d_{f}$ with respect to the anisotropy factor $b$, when $a=1$. The lines correspond to the theory predicted by Eq. (5.32) and the dots are the average distance to the first caustics from numerical simulations. We see an excellent agreement between numerics and analytics. The deviations for the small values of $b$ and $d_{f}$ are a numerical artifact. Indeed, when $b$ is very small, the effective correlation length in the propagation direction can sometimes be very long (for the points with short $d_{f}$ ), meaning that there are not enough correlation lengths per potential, as we produce a small potential that we repeat in periodic paving. The potential therefore does not have the exact correlation function that we inserted. The units for the distance are arbitrary.

Furthermore, we will also verify that the height fluctuations of shallow water waves in anisotropic random bathymetries follow the predictions of the ray calculations. As those simulations are computationally very demanding, we only looked at the angular distribution around point sources with a fixed $b=0.5$ and compared the results to the isotropic case $b=1$. Instead of using the scintillation index integrated in the transverse as in section 4.5 , we used a scintillation index that integrates the intensities over the realizations, in $2 \mathrm{D}$. The reason for this is that we cannot integrate over the angles anymore when dealing with the peanut-shape described in the ray case. This scintillation index is computed over the $n$ realizations with intensity $I_{n}(x, y)$ as follows

$$
\Sigma(x, y)=\frac{\frac{1}{n} \sum_{j=1}^{n} I_{j}^{2}(, x, y)-\bar{I}^{2}(x, y)}{\bar{I}^{2}(x, y)},
$$




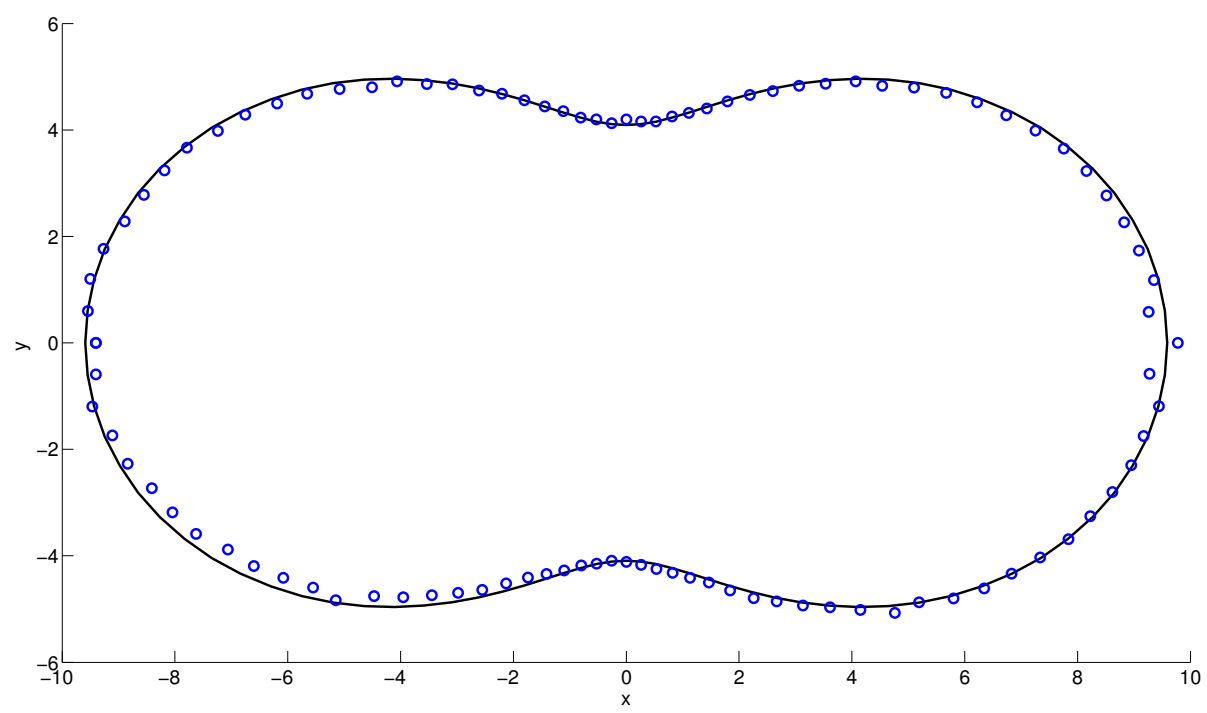

Figure 5.6: Here we have the polar distribution for the distance to the first caustics in anisotropic potentials for water rays, with $b=0.6$ and $a=1$, in the same fashion as in Fig 5.3 . The dots are of course the average distance to the first caustics from the numerical experiments and the black line is the theory from Eq. (5.18). Once again, there is excellent agreement. The deviations at the extremes could be explained by a lack of points for the statistics or a numerical artifact in the counting of the caustics via our binning system. The slight overall shift indicates a small error in the binning system. The units for the distance are arbitrary.

where $\bar{I}(x, y)$ is the average intensity at the position $(x, y)$. We ran wave simulations in 200 randomly generated isotropic bathymetries $(b=1)$, with a fixed standard deviation $\epsilon=0.08$ and correlation length $l_{c}=100 \mathrm{~km}$, and computed the two-dimensional scintillation index, which is ensemble averaged over the realizations, according to Eq. (5.33). The result is plotted in Fig 5.7 and we can see that the highest fluctuations are concentrated around a nice circle. We integrated this scintillation index over the angles in order to obtain the average radius of the first peak of the scintillation index, which gave us the missing prefactor for the scaling law, that we used in the anisotropic plot in Fig 5.8 , which was produced in the same fashion. We can see that the predicted angular distribution for the distance to the highest fluctuations (the red peanut-shaped curve) lies in the region were the scintillation index is maximal. This confirms that our theory for anisotropic potentials also applies to tsunami waves.

\subsection{Correlation on several length-scales}

We want to see how a multiple length-scale potential would affect the average distance to the first caustics. Of course, most systems could probably be well-approximated with only a handful of length-scales, but we will derive a formula for an arbitrary number of correlation lengths and then look at more practical examples. We choose to model the various correlations with distinct correlation functions that we will then sum to obtain the overall correlation function, as follows

$$
c(x, y)=\frac{1}{N} \sum_{i=1}^{n}(-1)^{s_{i}} \epsilon_{i}^{2} f_{i}\left(r^{2} / l_{i}^{2}\right),
$$

with $n$ the number of length-scales, $r^{2}=x^{2}+y^{2}$ for isotropy, $\epsilon_{i}$ the strength of the correlation on the length-scale $l_{i}$ with correlation function $f_{i}\left(r^{2} / l_{i}^{2}\right)$ and $s_{i} \in\{0,1\}$ the parameter that determines whether there is correlation or anti-correlation on the associated length-scale $l_{i}$. 


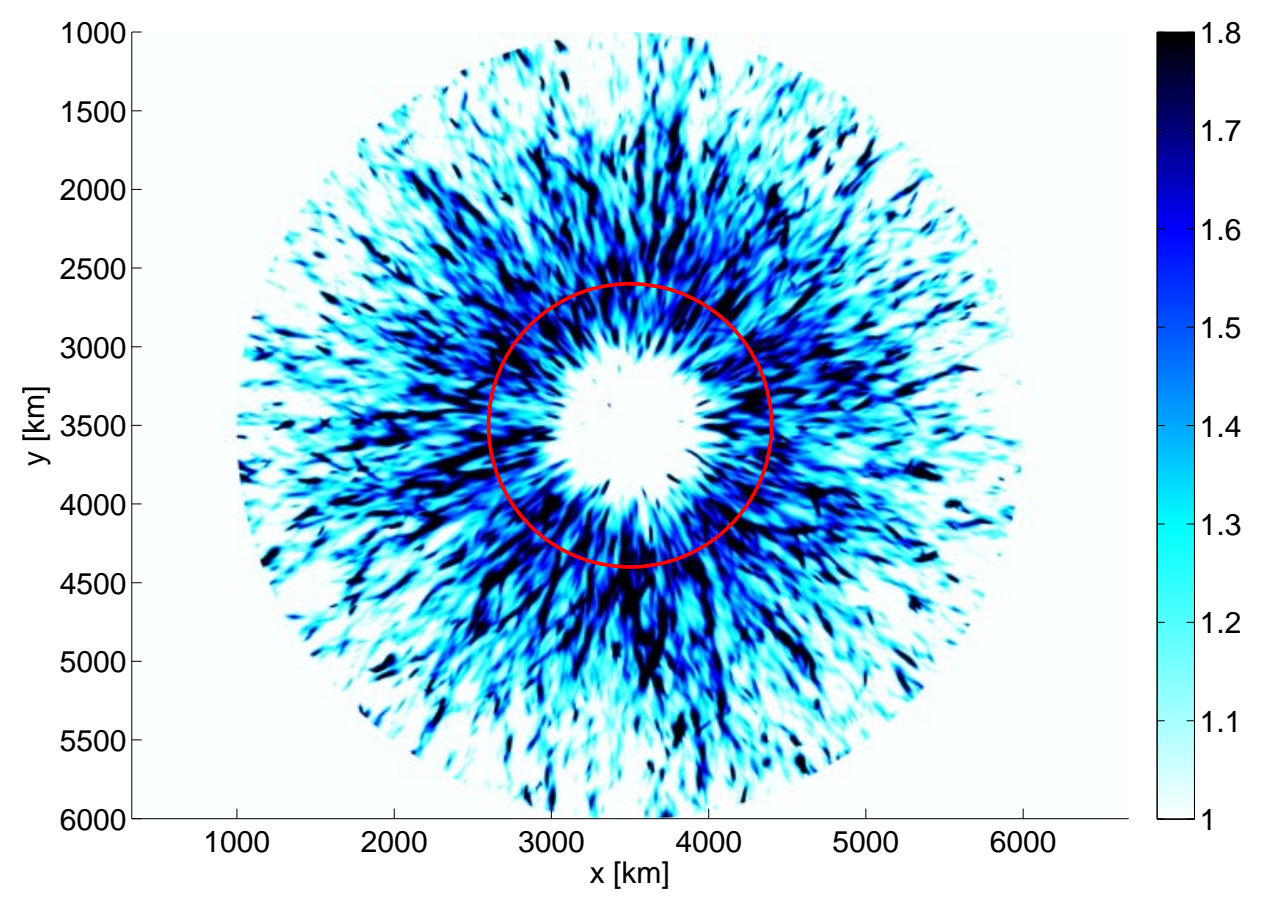

Figure 5.7: In shades of blue is the 2D scintillation index computed using Eq. 5.33) over 200 realizations for the bathymetry. We see that the location where it peaks forms a circle, that fits very well with our theoretical prediction from chapter 4 , which is shown in red.

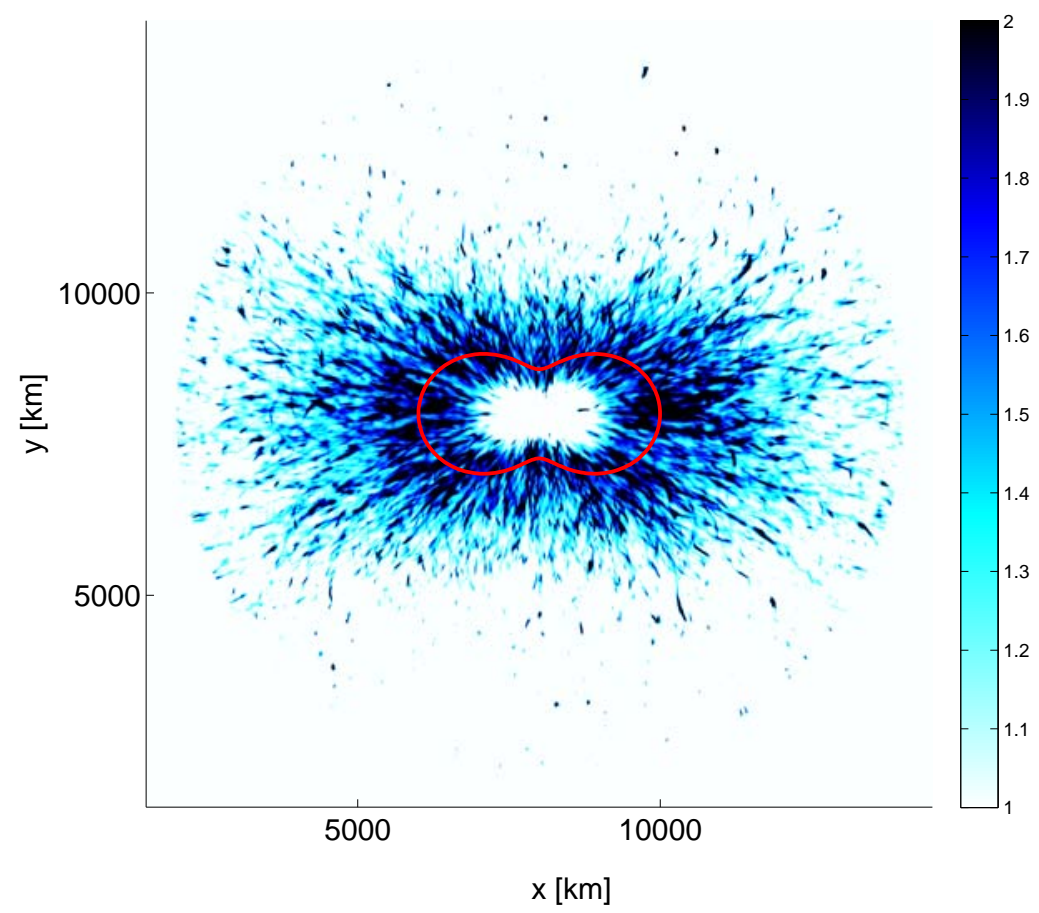

Figure 5.8: In shades of blue is the 2D scintillation index computed using Eq. (5.33) over 200 realizations for the bathymetry. We see that the location where it peaks forms the typical peanut shape discussed in the previous section that fits very well with our theoretical prediction, which is shown in red. 
Indeed, it is fairly common to see potentials or bathymetries that are positively correlated on some scales and then start to be anti-correlated (so, negatively correlated) on others. As in previous sections, $f_{i}: \mathbb{R} \longrightarrow \mathbb{R}$ has to be four times differentiable and we require similar convergence properties for some integrals, as in Eq.(5.5) and Eq.(5.39). $N$ is a normalization factor that ensures that $c(0,0)=\epsilon^{2}$ (see a little later when all $\epsilon_{i}$ are given in terms of a single $\epsilon$ and some relative strength factor $\gamma_{i}$, as described in Eq.(5.41)).

We have to be careful with this formulation, though, as some choices of parameters could lead to functions that cannot be correlation functions. Indeed, we have to remember that, originally, the random potential is the physical object from which we extract a correlation function, not the other way around. The way we produce potentials (which is explained in appendix A) is somewhat artificial, in that sense. First, we have to be sure that the correlation is maximal and positive at the origin. Anything different would be non-sense, because it would mean that there are locations which are more correlated to other locations in the potential than to themselves. Second, the Fourier transform of the correlation function must be non-negative, because the Wiener-Khinchin theorem states that the Fourier transform of the auto-correlation is the power spectrum of the potential [55], which is an integral over a function squared.

As in section 5.1, the only thing that we change here, compared to the simpler cases presented in chapter 2 and 4 , is the correlation function, which means that the main change is going to come from the $\sigma_{m}$, which is again defined in the following fashion

$$
\sigma_{m}^{2}=\left.\frac{1}{2} \int_{-\infty}^{+\infty} \mathrm{d} x\left[\partial_{y}^{2 m} c(x, y)\right]\right|_{y=0},
$$

where again $y$ is the transverse direction. Since we are working here with isotropic potentials, this choice is trivial. Considering our model in Eq.(5.34), we can write

$$
\sigma_{m}^{2}=\left.\frac{1}{2 N} \sum_{i=1}^{n}(-1)^{s_{i}} \epsilon_{i}^{2} \int_{-\infty}^{+\infty} \mathrm{d} x\left[\partial_{y}^{2 m} f_{i}\left(\frac{x^{2}+y^{2}}{l_{i}^{2}}\right)\right]\right|_{y=0} .
$$

In order to go forward, we need to consider the cases separately, as for the anisotropic case.

\subsubsection{Classical rays}

As in section 5.1.1, the curvature equation is what needs to be worked on and it involves $\sigma_{2}$. According to Eq. (5.36), we have

$$
\sigma_{2}^{2}=\left.\frac{1}{2 N} \sum_{i=1}^{n}(-1)^{s_{i}} \epsilon_{i}^{2} \int_{-\infty}^{+\infty} \mathrm{d} x\left[\partial_{y}^{4} f_{i}\left(\frac{x^{2}+y^{2}}{l_{i}^{2}}\right)\right]\right|_{y=0},
$$

which requires the fourth derivatives of the $f_{i}$ functions and that yields

$$
\left.\left[\partial_{y}^{4} f_{i}\left(\frac{x^{2}+y^{2}}{l_{i}^{2}}\right)\right]\right|_{y=0}=12 \frac{1}{l_{i}^{4}} f_{i}^{(2)}\left(x^{2} / l_{i}^{2}\right)
$$

where once again $f_{i}^{(n)}=\frac{d^{n}}{d z^{n}} f_{i}(z)$. We will require each of the following integrals to be convergent

$$
\int_{-\infty}^{+\infty} \mathrm{d} z f_{i}^{(n)}\left(z^{2}\right)=: \kappa_{n}^{i}<\infty
$$

such that we can write (after a change of variable $z=x / l_{i}$ )

$$
\sigma_{2}^{2}=\frac{6}{N} \sum_{i=1}^{n}(-1)^{s_{i}} \frac{\epsilon_{i}^{2}}{l_{i}^{3}} \kappa_{2}^{i}
$$


It would seem easier to write all the $\epsilon_{i}$ and $l_{i}$ using proportionality constants, in the following fashion

$$
\epsilon_{i}^{2}=\gamma_{i} \epsilon_{1}^{2} \quad \text { and } \quad l_{i}=\lambda_{i} l_{1} \quad, \quad \forall i \in\{2, . ., n\} .
$$

In this way, the parameters of the system are one main standard deviation $\epsilon_{1}$ and one main correlation length $l_{1}$ which we choose to be the shortest one without loss of generality (i.e. $\lambda_{i} \geq 1, \forall i>1$ ), along with the relative strength of the corresponding parameters in the other functions, which are then dimensionless. With this, we can finally write

$$
\sigma_{2}^{2}=\frac{6 \epsilon_{1}^{2}}{N l_{1}^{3}}\left((-1)^{s_{1}} \kappa_{2}^{1}+\sum_{i=2}^{n}(-1)^{s_{i}} \frac{\gamma_{i} \kappa_{2}^{i}}{\lambda_{i}^{3}}\right)
$$

This is the general result for $\sigma_{2}$ in multi-length-scale potentials, according to our model in Eq. (5.34). The only thing that we need to do then is to insert this expression in our previous result for the distance to the first caustics.

As an example, we consider the simpler case of two length-scales and two Gaussian correlated fields with $s_{1}=0$ and $s_{2}=1$, which yields

$$
\sigma_{2}^{2}=\frac{6 \sqrt{\pi} \epsilon^{2}}{N l^{3}}\left(1-\frac{\gamma}{\lambda^{3}}\right),
$$

where $N=1-\gamma$ in this case.

We note that cases where the contents of the brackets in Eq. (5.43) go to zero or negative values are prohibited by the fact that the power spectrum needs to be non-negative, as is explained at the beginning of section 5.2. Indeed, we can prove it by looking at the Fourier transform of the correlation function to find the power spectrum and impose a constraint on the parameters. The correlation function is given by

$$
c(x, y)=\frac{\epsilon^{2}}{1-\gamma}\left[e^{-\frac{x^{2}+y^{2}}{l_{c}^{2}}}-\gamma e^{-\frac{x^{2}+y^{2}}{\lambda^{2} l_{c}^{2}}}\right]
$$

so its Fourier transform, which will be the power spectrum of the associated potential, can be written as

$$
F(\vec{k})=\frac{\epsilon^{2}}{2 \pi(1-\gamma)} \int_{\mathbb{R}^{2}} \mathrm{~d} \vec{x}\left[e^{-\frac{x^{2}+y^{2}}{l_{c}^{2}}}-\gamma e^{-\frac{x^{2}+y^{2}}{\lambda^{2} l_{c}^{2}}}\right] e^{i k_{x} x+i k_{y} y} .
$$

With the change of variable $\vec{x} \rightarrow l_{c} \vec{x}$ in the first two integrals and $\vec{x} \rightarrow l_{c} \lambda \vec{x}$ in the last two integrals and using the fact that the integrals in $x$ and $y$ are separable, we can write

$$
F(\vec{k})=\frac{\epsilon^{2} l_{c}^{2}}{2 \pi(1-\gamma)}\left[\int_{\mathbb{R}} \mathrm{d} x e^{-x^{2}} e^{i k_{x} x l_{c}} \int_{\mathbb{R}} \mathrm{d} y e^{-y^{2}} e^{i k_{y} y l_{c}}-\gamma \lambda^{2} \int_{\mathbb{R}} \mathrm{d} x e^{-x^{2}} e^{i k_{x} x l_{c} \lambda} \int_{\mathbb{R}} \mathrm{d} y e^{-y^{2}} e^{i k_{y} y l_{c} \lambda}\right] .
$$

We deal with these integrals in the following way. Let us compute the first one

$$
\int_{\mathbb{R}} \mathrm{d} x e^{-x^{2}} e^{i k_{x} x l_{c}}=\int_{\mathbb{R}} \mathrm{d} x e^{-\left(x-\frac{i}{2} k_{x} l_{c}\right)^{2}-\frac{1}{4} k_{x}^{2} l_{c}^{2}}=e^{-\frac{1}{4} k_{x}^{2} l_{c}^{2}} \int_{\mathbb{R}} \mathrm{d} x e^{-\left(x-\frac{i}{2} k_{x} l_{c}\right)^{2}}=\sqrt{\pi} e^{-\frac{1}{4} k_{x}^{2} l_{c}^{2}},
$$

where the last equality comes from the fact that even complex Gaussians have the same integral as real Gaussians, due to Cauchy's theorem. Each of these four integrals can be treated in the same way, such that we can write

$$
F(\vec{k})=\frac{\epsilon^{2} l_{c}^{2}}{2(1-\gamma)}\left[e^{-\frac{1}{4} l_{c}^{2} k^{2}}-\gamma \lambda^{2} e^{-\frac{1}{4} \lambda^{2} l_{c}^{2} k^{2}}\right],
$$


where $k^{2}=k_{x}^{2}+k_{y}^{2}$. Calling the first Gaussian $G(k)=e^{-\frac{1}{4} k^{2} l_{c}^{2}}$, we find

$$
F(\vec{k})=\frac{\epsilon^{2} l_{c}^{2}}{2(1-\gamma)} G(k)\left[1-\gamma \lambda^{2}[G(k)]^{\lambda^{2}-1}\right] .
$$

As previously stated, the Wiener-Khinchin theorem says that $F(k)$ is the power spectrum of the random potential associated with our correlation function and therefore needs to be non-negative. As $0 \leq G(k) \leq 1$ because it is a Gaussian, we have to find a condition on $\gamma$ and $\lambda$ such that

$$
(1-\gamma)^{-1}\left(1-\gamma \lambda^{2}[G(k)]^{\lambda^{2}-1}\right) \geq 0 .
$$

With $\lambda \geq 1$, the worst case is when $G(k)=1$, which leads to

$$
(1-\gamma)^{-1}\left(1-\gamma \lambda^{2}\right) \geq 0 .
$$

There are two possible cases here. First, we can have $(1-\gamma)>0$ (i.e. $\gamma<1$ ), which leads to

$$
\gamma \lambda^{2} \leq 1
$$

For $\sigma_{2}^{2}$ to become negative in Eq. 5.43, we would need

$$
\left(1-\frac{\gamma}{\lambda^{3}}\right)<0 \text {. }
$$

This is equivalent to

$$
1<\frac{\gamma}{\lambda^{3}}=\frac{\gamma \lambda^{2}}{\lambda^{5}} \leq \frac{1}{\lambda^{5}}<1
$$

which is a contradiction, therefore showing that Eq.5.53 is never true. The second inequality comes from Eq. 5.52 and the last one comes from our hypothesis of $\lambda \geq 1$.

For the second case, i.e. $\gamma \geq 1$, we have

$$
\gamma \geq \frac{1}{\lambda^{2}}
$$

which is automatically fulfilled as $\lambda \geq 1$. Generalizing this argument to the general formula would give some very complicated expression that would probably be too intricate to be of any use, but we hypothesize that this argument is generally valid.

Now that we have made sure that $\sigma_{2}^{2}$ is always positive for potentials that make sense, we can find the theoretical distance to the first caustics

$$
d_{f}=C_{s}^{c}\left(\frac{(1-\gamma)}{6 \sqrt{\pi}}\right)^{1 / 3} \epsilon^{-2 / 3} l\left(1-\frac{\gamma}{\lambda^{3}}\right)^{-1 / 3} .
$$

There is of course no way to actually verify the general result numerically, as the number of parameters in potentially boundless. Instead, we want to test numerically the result obtained in Eq. 5.56). Because of condition Eq. 5.52), we decided to vary the combination $\gamma \lambda^{2}$ instead of $\gamma$, such that it is easier to satisfy the said condition. The result can be seen in Fig 5.9 and shows an excellent agreement with the theory. Even if we cannot show it in the general case, we take this successful prediction as a good sign that our theory is valid.

\subsubsection{Water rays}

We now want to apply a similar theory to the case of the water waves and their associated rays. As for the anisotropic case, the derivation will be slightly different than for the rays, as the argument leading to the scaling law works differently. We can, however, mimic the process we developed in section 5.1.2 to find the distance to the first caustics. Again, we will start from the variance in the transverse direction, that was given in Eq.5.31) by

$$
\left\langle y^{2}(t)\right\rangle \approx \frac{1}{6} c_{0}^{4} \sigma_{1}^{2} t^{3},
$$




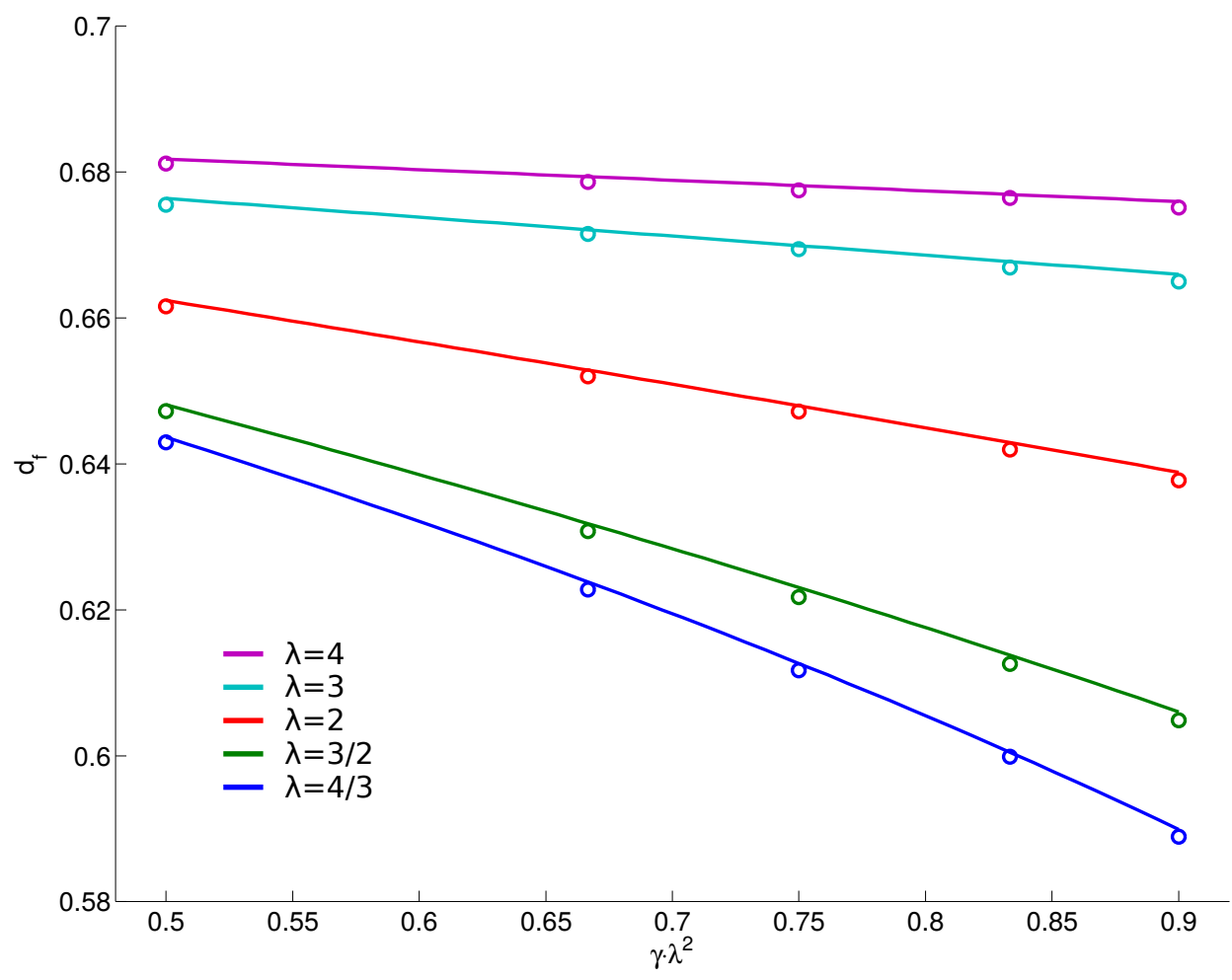

Figure 5.9: The scaling of the distance to the first caustics with respect to the product $\gamma \lambda^{2}$ for various values of $\lambda$, here for classical rays. We see that the plain lines representing the theoretical expectation given by Eq. (5.56) fit the numerical values (dots) quite well. For this fit, we had to slightly shift the theoretical prefactor from $C_{p w}^{c}=3.31$ to $C_{p w}^{c}=3.23$, meaning that there is a $2.5 \%$ deviation. Still, the functional dependency is very well predicted. The units for the distance are arbitrary.

where we can now use the new $\sigma_{1}$, which can be obtained with Eq.(5.36) and a straightforward computation

$$
\sigma_{1}^{2}=\frac{1}{N} \sum_{i=1}^{n}(-1)^{s_{i}} \frac{\epsilon_{i}^{2}}{l_{i}} \kappa_{1}^{i}
$$

Now, we want to use the same argument as before, which was already used in Eq. 5.30 and reads

$$
\left\langle y^{2}\left(t_{f}\right)\right\rangle \sim l_{e}^{2},
$$

where $l_{e}$ is an effective correlation length in the transverse direction. We actually do not know how to produce a general formula for this effective correlation length. However, we expect each correlation length $l_{i}$ to have a sort of weight in the expression for $l_{e}$, since they are associated with correlations of different strengths. We believe the best approach here is to try to understand the simpler case already discussed at the end of the previous section, with two Gaussian correlated contributions, with one anti-correlated, leading to

$$
\sigma_{1}^{2}=\frac{\sqrt{\pi} \epsilon^{2}}{(1-\gamma) l}\left(1-\frac{\gamma}{\lambda}\right)
$$

As we were not sure about how to attack this problem and we had a strong suspicion that the scaling would be exactly the same as for classical rays (since, so far, it has always been the same, up to the prefactors), we decided to run simulations first to verify our intuition. We plot the 
result in Fig 5.10 , where we used the empirical prefactor for water rays $C_{p w}^{w}=2.55$ determined in chapter 4 instead of the prefactor for classical rays. We see that the fit is incredibly good. Now, we can compute $l_{e}$ starting from this result. Indeed, we have, on the one hand, the result from

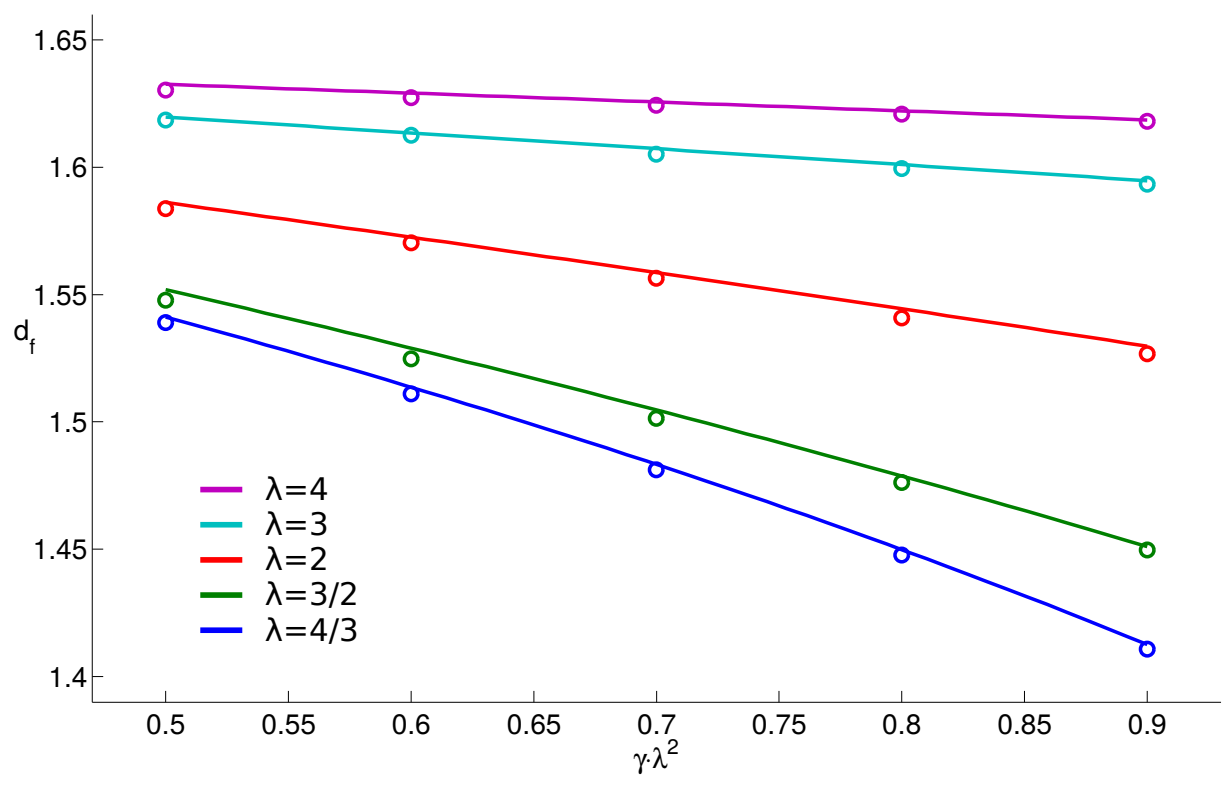

Figure 5.10: The scaling of the distance to the first caustics $d_{f}$ with respect to the product $\gamma \lambda^{2}$ for various values of $\lambda$, in the case of water rays. We see that the plain lines representing the theoretical expectation given by Eq.(5.56) fit the numerical values (dots) quite well. For this fit, we had to slightly shift the theoretical prefactor from $C_{p w}^{c}=2.55$ to $C_{p w}^{c}=2.57$, meaning that there is a $1 \%$ deviation, which shows a rather impressive agreement. The units for the distance are arbitrary.

the numerics

$$
d_{f, \text { numerics }}=C_{s}^{w}\left(\frac{6(1-\gamma)}{\sqrt{\pi}}\right)^{1 / 3} \epsilon^{-2 / 3} l\left(1-\frac{\gamma}{\lambda^{3}}\right)^{-1 / 3}
$$

and on the other hand, our argument suggests that we have

$$
\sigma_{1}^{2} d_{f, \text { theory }}^{3} \sim l_{e}^{2} .
$$

We can now compare $d_{f \text {,numerics }}=d_{f, \text { theory }}$ in order to figure out what is actually $l_{e}$. This yields (once we divided out all the prefactors)

$$
d_{f, \text { numerics }} \sim \epsilon^{-2 / 3} l(1-\gamma)^{1 / 3}\left(1-\frac{\gamma}{\lambda^{3}}\right)^{-1 / 3}=l_{e}^{2 / 3} l^{1 / 3} \epsilon^{-2 / 3}(1-\gamma)^{1 / 3}\left(1-\frac{\gamma}{\lambda}\right)^{-1 / 3} \sim d_{f, \text { theory }} .
$$

We can divide out the common factors and reorganize the terms to obtain

$$
l_{e}^{2 / 3}=l^{2 / 3}\left(1-\frac{\gamma}{\lambda^{3}}\right)^{-1 / 3}\left(1-\frac{\gamma}{\lambda}\right)^{1 / 3},
$$

which can be simplified into

$$
l_{e}=l \sqrt{1+\gamma \frac{1-\lambda^{2}}{\lambda^{3}-\gamma}} .
$$

As of now, we do not know how to interpret this effective correlation length, as the correction in the square root (which is what distinguishes this $l_{e}$ from the main $l$ ) looks relatively intricate. 
Even though we cannot understand this scaling law theoretically, we believe that it is useful to verify if this empirical scaling law given in Eq.(5.61) still holds for waves. The result can be seen in Fig. 5.11 .
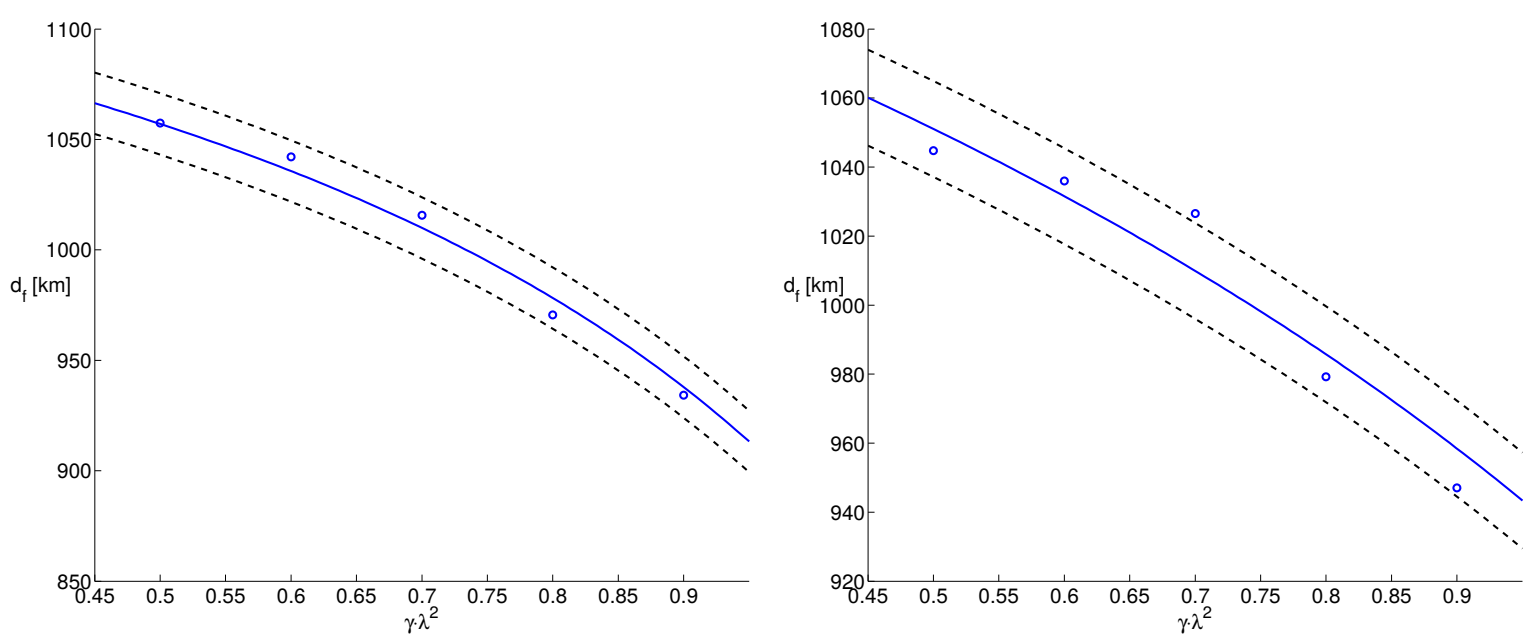

Figure 5.11: Two scaling curves with $\lambda=1.15$ on the left and $\lambda=1.3$ on the right. For each curve, we have a fitted theoretical curve from Eq.(5.56) in plain blue (we fitted the prefactor) and two dashed curves corresponding to the fitted curve \pm one wavelength. Even if there are slight deviations, we can see an overall good agreement between the numerical data point (dots) and the theoretical scaling. These deviations could be due to a lack of statistics and resolution issues in the creation of the potential.

\subsection{Conclusion}

In this section, we investigated how more complicated random media would affect the statistics of random caustics and branched flows by computing the distance to the first caustics in two main cases, that we decided to separate for the sake of clarity.

Globally anisotropic potentials bring a characteristic angular dependence (the peanut shape) for the distance to the first caustics, both for classical rays and water waves. Even though the argument used in the derivations were quite different, we found again the same scaling law for both cases.

Potentials that are correlated on several length scales proved to be more challenging, as we were unable to actually find a good argument in the case of the water waves. We did, however, show that the scaling law is the same for classical rays and water waves with extensive numerical simulations.

Although we have decided not to include it here, we are confident that the combination of these two types of correlations is a straightforward extension of the theory presented here and we leave this calculation to the interested reader. The general expression that we expect for such cases is intricate and we believe that it wouldn't be very illuminating, considering what was already shown here.

This chapter closes the main body of this thesis, which was the theoretical study of the caustic statistics in tsunami waves. In the next chapter, we will present some theoretical estimates that we calculated in the prospect of an experimental laboratory study of the focusing of tsunami waves. 


\section{Chapter 6}

\section{Can we study tsunami focusing in the laboratory?}

Our study, so far, has been based on theoretical arguments and has been confirmed with numerical simulations. As valuable as these methods are, there is no better way to confirm the relevance of a result than with an experiment.

We want to find a way to verify our results in a real system, which has the same behavior as a tsunami wave, but can fit in a laboratory. This means that we need to scale down our system to a size ranging from the centimeter scale to the meter scale. However, the use of water is out of the question, as the viscosity and surface tension can become problematic, as will be shown below. Fortunately, our institute is equipped with a high pressure facility, which allows us to consider the use of liquified gases. The experimentalists suggested the use of sulfur hexafluoride $S F_{6}$, the surface tension and viscosity coefficients of which are substantially smaller than of water.

The purpose of this chapter is to first lay down the theoretical basis of this experiment and give the relevant estimates that justify our approach. Afterwards, we will present the actual concept of the setup we intend to use for the experiment, which at the time of the writing is still under preparation.

As the idea is to scale down a tsunami wave to the size of our container, the latter will of course be the main restrictive parameter of our experimental setup. The setup we will use for the first exploratory experiment provides a maximal propagation distance of about 10 centimeters. Even when considering a single deterministic scatterer, we need at least five to ten wavelengths in order to see a proper focusing pattern. This means that our wavelength can be at best 2 centimeters long. From [58], we know that the shallow water approximation is reasonable for wavelengths larger than 3 times the depth of the fluid. In order to make sure that we are in the shallow water regime, we will restrict this ratio to be larger than 5 , meaning that the fluid depth should be at most 0.4 centimeter. Next we want to discuss the restrictions to the amplitudes of the waves. Throughout this works, we have been working with the linearized shallow water equations, which apply very well to tsunamis. However, we have to keep in mind that they are only valid if the amplitude, compared to the depth of the fluid, is small enough. For real tsunamis, this ratio is between $10^{-3}$ and $10^{-4}$. Considering that we would apply such a ratio to our experiment, this would lead to waves with micrometer-high amplitudes, which seems very difficult to measure. Estimating the error made by using the linear approximation is not straightforward, as it requires one to solve the nonlinear shallow water equations. In order to investigate this, we produced a few one-dimensional nonlinear wave simulations and compared them to linear simulations, to see at what point the nonlinearity started to be important enough to produce a substantial change in the waves. We are aware that this is a hand-waving method, but the idea was to obtain a quick estimate on that matter. We observed that the waves show almost no differences between the linear and nonlinear simulations, as long as the amplitude of the wave was below $5 \%$ of the depth of the fluid. To be on the safe side, we will consider $2.5 \%$ of 
the depth of the fluid, which leads to $100 \mu \mathrm{m}$ in amplitude. With this in mind, we provide, in Tab.6.1, the numerical values for the parameters of the experiment setup that will be presented later in this chapter, along with the values for tsunami waves, for comparison purposes.

\begin{tabular}{|c|c|c|c|}
\hline & & Tsunami & $S F_{6}$ \\
\hline Average depth & $H_{0}$ & $4 \cdot 10^{3} \mathrm{~m}$ & $4 \cdot 10^{-3} \mathrm{~m}$ \\
\hline Surface tension coefficient & $\sigma$ & $7.2 \cdot 10^{-2} \mathrm{~N} / \mathrm{m}$ & $6 \cdot 10^{-5} \mathrm{~N} / \mathrm{m}$ \\
\hline Viscosity coefficient & $\mu$ & $9 \cdot 10^{-4} \mathrm{~Pa} \cdot \mathrm{s}$ & $6 \cdot 10^{-5} \mathrm{~Pa} \cdot \mathrm{s}$ \\
\hline Fluid density & $\rho$ & $10^{3} \mathrm{~kg} / \mathrm{m}^{3}$ & $0.982 \cdot 10^{3} \mathrm{~kg} / \mathrm{m}^{3}$ \\
\hline Wavelength & $\lambda$ & $10^{5} \mathrm{~m}$ & $2 \cdot 10^{-2} \mathrm{~m}$ \\
\hline
\end{tabular}

Table 6.1: The numerical values for our two fluids. Water is given at normal conditions $\left(25^{\circ} \mathrm{C}, 1\right.$ bar) for tsunamis and $S F_{6}$ is given at tank conditions ( $44^{\circ} \mathrm{C}$, 36.2 bars).source: [70]

\subsection{Dispersion relation}

When studying wave equations, it is often useful to consider a quantity called the dispersion relation $\omega(k)$, which gives the dependency of the frequency $\omega$ of the wave with respect to the wavenumber $k$, which is directly related to the wavelength $\lambda=2 \pi / k$. Typically, we would look at the linearized shallow water wave equation with a flat bathymetry

$$
\left(\partial_{t}^{2}-c_{0}^{2} \Delta\right) \eta(\vec{x}, t)=0
$$

and use a wave ansatz

$$
\eta(\vec{x}, t)=A e^{i \vec{k} \cdot \vec{x}+i \omega t} .
$$

Inserting Eq.66.2) into Eq.66.1) yields

$$
\left(\omega^{2}-c_{0}^{2} k^{2}\right) e^{i \vec{k} \cdot \vec{x}+i \omega t}=0 \longrightarrow \omega_{ \pm}(k)= \pm c_{0} k,
$$

where $k=\sqrt{\vec{k} \cdot \vec{k}}$. This is the dispersion relation for linear shallow water waves, which we will refer to as gravity waves, since they are only generated by the force of gravity.

The idea now is to add viscosity and surface tension terms to the shallow water equations in order to see how much they modify the dispersion relation, i.e. to see how strong the extra terms will be compared to the gravity term. For the sake of simplicity and keeping in mind that our objective is to make estimates, we will consider viscosity and surface tension separately.

\subsection{Surface tension}

As its name suggest, the surface tension is an effect that acts on surfaces and is associated with minimizing the tensile energy, which can be understood in terms of the curvature of the surface, e.g. soap bubbles tend to form spheres. According to [71], the surface tension can be modeled through a pressure term at the interface of the fluid with the atmosphere that is proportional to the second derivatives of the surface, i.e. the curvature. This means that, compared to what was done in chapter 3 , the pressure at the surface $h(x, y, t)$ of the fluid won't be set to zero, but instead will be written as

$$
\left.p(x, y, z, t)\right|_{z=h}=-\sigma \Delta h(x, y, t) .
$$

As a reminder of the quantities used in the derivation, we show again the scheme of chapter 3 in Fig. 6.1. Considering the new pressure terms from Eq. 6.4], the overall pressure from the hydrostatic equilibrium hypothesis now reads

$$
\left.p(x, y, z, t)\right|_{z=h}=\rho g(h(x, y, t)-z)-\sigma \Delta h(x, y, t) .
$$




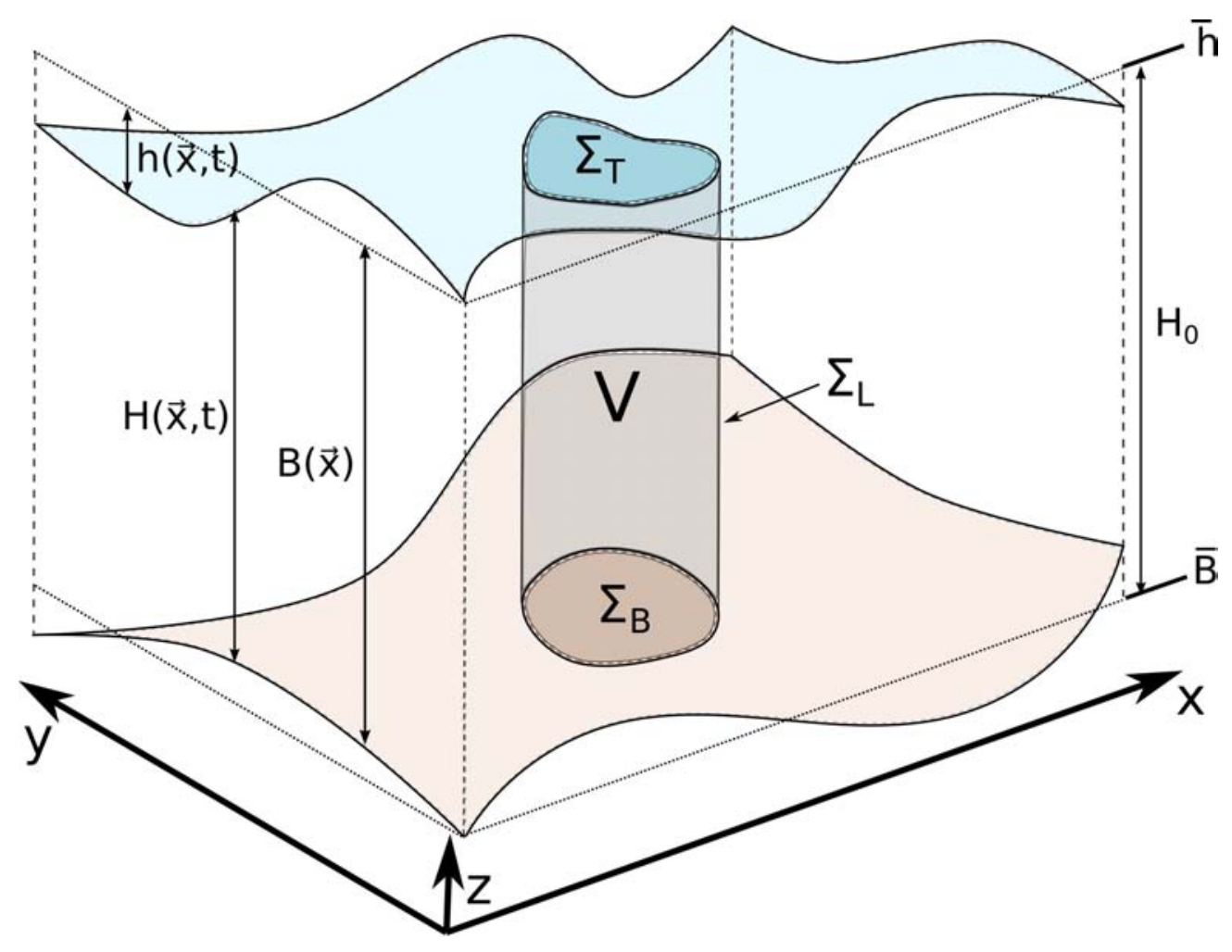

Figure 6.1: This is a scheme of the fluid under consideration. The bottom sheet (brown) represents the floor (bathymetry) and the top sheet (blue) represents the surface of the fluid. The volume V is framed by the bottom surface $\Sigma_{B}$, the top surface $\Sigma_{T}$ and the lateral surface $\Sigma_{L}$. The values $\bar{h}$ and $\bar{B}$ are respectively the average level of the surface of the fluid (its position at rest) and the average elevation of the floor. The difference between these two values therefore defines the average depth $H_{0}$. The function $h(x, y, t)$ describes the surface elevation. $B(x, y)$ is the bathymetry, measured from $\bar{h} . H(x, y, t)=h(x, y, t)-B(x, y)$ is the actual height of the fluid column. Here $\vec{x}$ stands for the horizontal space $(x, y)$.

Considering that the only change compared to the simple gravity case is in the pressure, the mass conservation equation does not change, which means that the first equation from the linearized shallow water equations stays the same

$$
\partial_{t} \eta=-\nabla \cdot[(1-\beta) \vec{u}]
$$

where we remind the reader that we use the dimensionless quantities $\eta(x, y, t)=H_{0}^{-1} h(x, y, t)$ and $\beta(x, y)=H_{0}^{-1}\left[B(x, y)+H_{0}\right]$. The interesting thing comes in the momentum equations, that were written

$$
\partial_{t} \int_{V} \rho \vec{u}_{\perp} \mathrm{d} V=-\int_{\Sigma_{L}}\left(\rho \vec{u}_{\perp}\right) \vec{u} \cdot \vec{n} \mathrm{~d} S-\int_{\Sigma_{L}} p(x, y, z, t) \vec{n} \mathrm{~d} S-\int_{\Sigma_{B}} p(x, y, z, t) \vec{n}_{\perp} \mathrm{d} S-\int_{\Sigma_{T}} p(x, y, z, t) \vec{n} \mathrm{~d} S,
$$

where the same terminology as in chapter 3 applies. We note that there is now an extra pressure term at the top boundary because the pressure is no longer zero there. All the terms are treated as in chapter 3 and each pressure integral contains a new term 


$$
\begin{aligned}
\int_{\Sigma_{T}} p(x, y, z, t) \vec{n} \mathrm{~d} S & =\int_{\Sigma_{T}}(\rho g(h(x, y, t)-z)-\sigma \Delta h(x, y, t)) \vec{n} \mathrm{~d} S \\
& =\int_{D} \sigma \Delta h(x, y, t) \vec{\nabla}_{\perp} h \mathrm{~d} S, \\
\int_{\Sigma_{L}} p(x, y, z, t) \vec{n} \mathrm{~d} S & =\int_{\Sigma_{L}}(\rho g(h(x, y, t)-z)-\sigma \Delta h(x, y, t)) \vec{n} \mathrm{~d} S \\
& =\oint_{\partial D}\left[\frac{1}{2} \rho g H^{2}-H \sigma \Delta h(x, y, t)\right] \vec{n}_{\perp} \mathrm{d} \gamma \\
\int_{\Sigma_{B}} p(x, y, z, t) \vec{n} \mathrm{~d} S & =\int_{\Sigma_{B}}(\rho g(h(x, y, t)-z)-\sigma \Delta h(x, y, t)) \vec{n} \mathrm{~d} S \\
& =\int_{D} \rho g H-\sigma \Delta h(x, y, t) \vec{\nabla}_{\perp} B \mathrm{~d} S,
\end{aligned}
$$

where $\vec{n}$ is generally an outward unit vector normal to the surface of integration, $\vec{n}_{\perp}$ is the unit vector pointing in the outward direction normal to $\partial D$, which is the curvilinear boundary of the domain $D$, which is the projection of $\Sigma_{T}$ (and $\Sigma_{B}$, by definition) on the xy-plane. For the first integral, at the surface, the hydrostatic pressure term is of course zero and the $\nabla_{\perp} h$ in the last equality comes from the reparametrization of the domain, as in the bottom integral (see chapter 3 ). For the second integral, we integrate over $z$ in order to get the integral over the edge of the domain $D$. We note here that, since the capillary pressure term is not proportional to $\rho$, it will get a $\rho^{-1}$ factor when we divide it out. Again, we can use Stokes theorem to put the surface and curvilinear integrals together in order to obtain the full shallow water equations with surface tension

$$
\begin{aligned}
\partial_{t} H+\partial_{x}(H u)+\partial_{y}(H v) & =0 \\
\partial_{t}(H u)+\partial_{x}\left(H u^{2}+\frac{1}{2} g H^{2}-\frac{\sigma}{\rho} H \Delta h\right)+\partial_{y}(H u v) & =-\left(g H-\frac{\sigma}{\rho} \Delta h\right) \partial_{x} B+\frac{\sigma}{\rho} \Delta h \partial_{x}(6.11 \mathrm{~b}) \\
\partial_{t}(H v)+\partial_{x}(H u v)+\partial_{y}\left(H v^{2}+\frac{1}{2} g H^{2}-\frac{\sigma}{\rho} H \Delta h\right) & =-\left(g H-\frac{\sigma}{\rho} \Delta h\right) \partial_{y} B+\frac{\sigma}{\rho} \Delta h \partial_{y}(16,11 \mathrm{c})
\end{aligned}
$$

As in chapter 3, we will reduce Eq. 6.11) to their linear and dimensionally simplified form using the expansion $h=H_{0}(1+\eta-\beta)$. After some simplifications, we obtain

$$
\begin{aligned}
\partial_{t} \eta & =-\nabla \cdot[(1-\beta) \vec{u}], \\
\partial_{t} \vec{u} & =-c_{0}^{2} \nabla \eta+\frac{\sigma}{\rho} H_{0} \nabla \Delta \eta,
\end{aligned}
$$

Once again, we can bring these equations back into a single wave equation for $\eta$, in the geometrical optics limit,

$$
\partial_{t}^{2} \eta=\left[c_{0}^{2}(1-\beta)-\frac{\sigma}{\rho} H_{0} \Delta\right] \Delta \eta .
$$

Now, in order to look at the dispersion relation, we set $\beta=0$ and we use the wave ansatz Eq. 6.2 which yields

$$
\left[\omega^{2}(k)-c_{0}^{2} k^{2}+\frac{\sigma}{\rho} H_{0} k^{4}\right]=0 \longrightarrow \omega_{ \pm}(k)= \pm c_{0} k \sqrt{1-\frac{\sigma}{g \rho} k^{2}} .
$$


So, in order to have a good approximation by neglecting the surface tension, we need the second term in the square root of Eq. 6.14) to be as small as possible, as it is the deviation from the simple gravity case. Using the numerical values from Tab.6.1, we find that real tsunamis are incredibly well-approximated when we neglect the surface tension, as we find

$$
\left(\frac{\sigma k^{2}}{g \rho}\right)_{\text {tsunami }}=\left(\frac{\sigma(2 \pi)^{2}}{g \lambda^{2} \rho}\right)_{\text {tsunami }} \simeq 2.8 \cdot 10^{-14}
$$

which is extremely small. On the other hand, the same ratio for water with the wavelength of 2 $\mathrm{cm}$ needed for the tank experiment would lead to a value of 0.7 , which is far above anything acceptable. It also fits our everyday experience that, on small scales, the behavior of water surface waves is observably affected by capillarity. For $S F_{6}$, however, we find

$$
\left(\frac{\sigma k^{2}}{g \rho}\right)_{S F_{6}}=\left(\frac{\sigma(2 \pi)^{2}}{g \lambda^{2} \rho}\right)_{S F_{6}} \simeq 1.5 \cdot 10^{-4}
$$

which, even though it is much higher than the real tsunami case, should still be small enough to allows us to neglect surface tension in the experiment, therefore expecting the wave behavior to be mainly associated with gravity waves.

\subsection{Viscosity}

Unlike the surface tension, the viscosity term, which is associated with friction and dissipation, cannot be derived from conservation laws, as it goes against the idea of conservation in its essence. In order to make our estimate, we will use the viscous shallow water equations, as given in $[72$ in one dimension

$$
\begin{aligned}
\partial_{t} H+\partial_{x}(H u) & =0, \\
\partial_{t}(H u)+\partial_{x}\left(H u^{2}+\frac{1}{2} g H^{2}\right) & =-g H \partial_{x} B+C H_{0} \partial_{x}\left(H \partial_{x} u\right),
\end{aligned}
$$

which we simply extend to two dimensions

$$
\begin{aligned}
\partial_{t} H+\partial_{x}(H u)+\partial_{y}(H v) & =0, \\
\partial_{t}(H u)+\partial_{x}\left(H u^{2}+\frac{1}{2} g H^{2}\right)+\partial_{y}(H u v) & =-g H \partial_{x} B+C \partial_{x}(H \nabla \cdot \vec{u}), \\
\partial_{t}(H v)+\partial_{x}(H u v)+\partial_{y}\left(H v^{2}+\frac{1}{2} g H^{2}\right) & =-g H \partial_{y} B+C \partial_{y}(H \nabla \cdot \vec{u}) .
\end{aligned}
$$

By dimensional analysis, we realize that the constant $C$ before the extra viscosity term is actually given by $C=\mu / \rho$, with $\mu$ the viscosity coefficient, as given in Tab.6.1, and $\rho$ is of course the density. Indeed, the units of the viscosity term are

$$
\left[C \partial_{x}(H \nabla \cdot \vec{u})\right]=[C] \cdot m^{-1} \cdot m \cdot m^{-1} \cdot m s^{-1}=[C] \cdot s^{-1},
$$

while the units of the l.h.s of the equation are

$$
\left[\partial_{t} H u\right]=s^{-1} \cdot m \cdot m s^{-1}=m^{2} s^{-2} .
$$

This means that $[C]=m^{2} s^{-1}$. Considering that we want it to be proportional to the viscosity factor $[\mu]=P a \cdot s=\mathrm{kg} \cdot \mathrm{m}^{-1} \mathrm{~s}^{-1}$, we find that the quantity that connects them is

$$
[C] \cdot[\mu]^{-1}=m^{3} \mathrm{~kg}^{-1}=[\rho]^{-1},
$$


which explains our conclusion that $C=\mu / \rho$.

As we did throughout this work, we want to study the linearized equations, using the expansion $H=H_{0}(1+\eta-\beta)$ for very small $\eta$ and $\vec{u}$, see chapter 3 . When we neglect all the nonlinear terms, we find

$$
\begin{aligned}
\partial_{t} \eta+\nabla[(1-\beta) \vec{u}] & =0, \\
\partial_{t} \vec{u}-\frac{\mu H_{0}}{\rho} \nabla[\nabla \cdot \vec{u}] & =-g H_{0} \nabla \eta .
\end{aligned}
$$

As in the previous section, we want to look at the dispersion relation, to quantify how much it diverges from the simple gravity case. For this, we consider a flat bathymetry $\beta=0$ and we look for a single wave equation for $\eta$, which is

$$
\partial_{t}^{2} \eta-\frac{\mu H_{0}}{\rho} \partial_{t} \Delta \eta-g H_{0} \Delta \eta=0 .
$$

Once again, we can use our wave ansatz from Eq. (6.2) to find

$$
\omega^{2}-\frac{i \mu H_{0}}{\rho} k^{2} \omega-g H_{0} k^{2}=0 .
$$

This is a simple second degree algebraic equation, which has the solution

$$
\omega_{ \pm}(k)=c_{0} k\left[\frac{i \mu H_{0} k}{2 \rho c_{0}} \pm \sqrt{1-\frac{\mu^{2} H_{0} k^{2}}{4 \rho g}}\right],
$$

where we remind the reader that we used $c_{0}=\sqrt{g H_{0}}$. So, there are two terms that we have to investigate, the one in the square root and the complex one. Let us start with the term in the square root. For the tsunami case, using the parameter values from Tab.6.1, we find

$$
\left(\frac{\mu^{2} H_{0} k^{2}}{4 \rho g}\right)_{\text {tsunami }}=3.2 \cdot 10^{-16},
$$

which, once again, explains why we do not need to consider viscosity in the real tsunami case. On the other hand, in our $S F_{6}$ experiment, we find

$$
\left(\frac{\mu^{2} H_{0} k^{2}}{4 \rho g}\right)_{S F_{6}}=3.6 \cdot 10^{-11}
$$

which is also very negligible.

The complex term, outside of the square root, accounts for the damping due to viscosity, bringing a term of the kind $e^{-t / \tau}$ in front of the oscillatory function. We will only be looking at its norm, to see if the damping has a significant effect or not. For the tsunami case, we have

$$
\left(\frac{\mu H_{0} k}{2 \rho c_{0}}\right)_{\text {tsunami }}=5.7 \cdot 10^{-10}=\tau^{-1}\left(c_{0} k\right)^{-1},
$$

leading to $\tau=1.4 \cdot 10^{11} \mathrm{~s}$, which is approximately 4404 years, so we can say that the damping is really negligible, considering that the typical period of a tsunami wave is of the order of tens of minutes and the propagation speed is around $700 \mathrm{~km} / \mathrm{h}$, meaning that the longest tsunamis might propagate for something like fifteen hours and therefore the damping will occur at the shores. Now, computing the same term in the $S F_{6}$ experiment, we obtain

$$
\left(\frac{\mu H_{0} k}{2 \rho c_{0}}\right)_{S F_{6}}=1.9 \cdot 10^{-7}
$$


leading to $\tau=8.4 \cdot 10^{4} \mathrm{~s}$, which is approximately 23 hours, meaning that it is also negligible, for the same reasons.

Interestingly, choosing water for the lab experiment wouldn't be so bad regarding viscosity, as the deviation from the gravity case (in the square root of Eq.(6.25)) would be around $8 \cdot 10^{-9}$ and the damping term (complex) would have a norm of $2.8 \cdot 10^{-6}$, both being perfectly negligible as well. It is however not surprising that the surface tension is much more of a problem than viscosity. Indeed, the main thing that changed here is the scale of the system, leading to changes in the wavelength and the depth of the fluid. These changes go towards an increase in the curvature of the surface, and thus the surface tension.

\subsection{Experimental setup}

\section{SIDE}

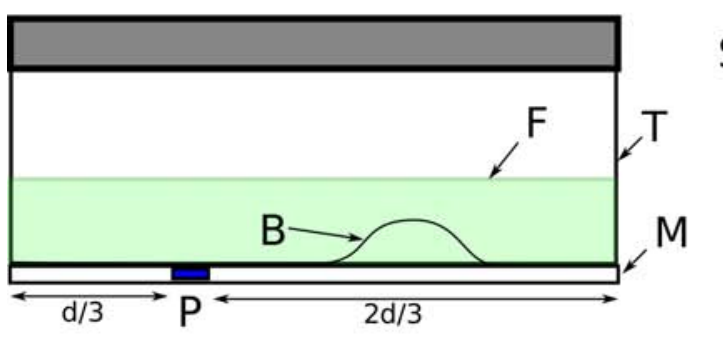

TOP

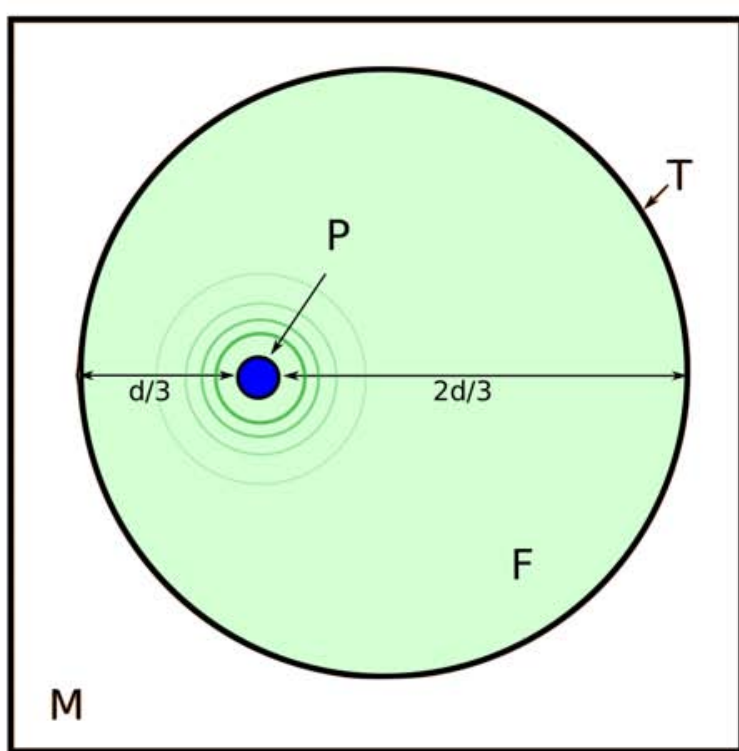

Figure 6.2: The left panel shows our experimental setup from the side and the right panel shows it from the top. Even though it is not very well represented here, we have to keep in mind that the whole setup is a high pressure tank. On the lateral view, we can see at the bottom the mirror $M$ that will reflect the light coming from the shadowgraph $S$ above, in the same fashion as in Fig 6.3 . The piezoelectric device $P$ that will be the source of the waves is mounted at the bottom of the container (for details on the piston, see Fig 6.4). The bathymetry structure is represented by the bump B. On the upper view, where we omitted the shadowgraph $S$ for obvious reasons, we can see the position of the source $P$ at $d / 3$, with $d$ being the diameter of the container. This was chosen to maximize the propagation distance before having backscattering issues. The rings around the source represent propagating waves.

We will first attempt to make a preliminary "proof of principle" experiment in a small high-pressure tank which has a diameter of around $15 \mathrm{~cm}$. The experiment will study the patterns in the surface waves focused by an isolated bathymetry structure, as described by Berry [6. The experimental setup is presented in Fig 6.2. We will excite waves on the surface with a small piston embedded in the floor of the container, which will be moved by a small piezoelectric crystal which can vibrate when we apply the appropriate voltage. Then, we let the wave propagate over a scatterer molded in the floor of the tank (as can be seen in Fig, 6.2) and we measure the intensity patterns on the surface. In order to do so, we will use a tool called a shadowgraph. The principle behind a shadowgraph is to project a beam of parallel light rays through a medium and measure the intensity of the light beam at some distance after it passed 
SKETCH

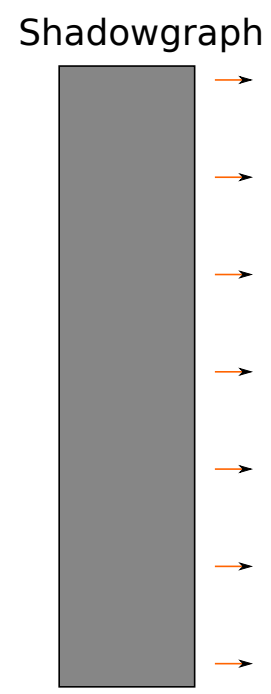

1

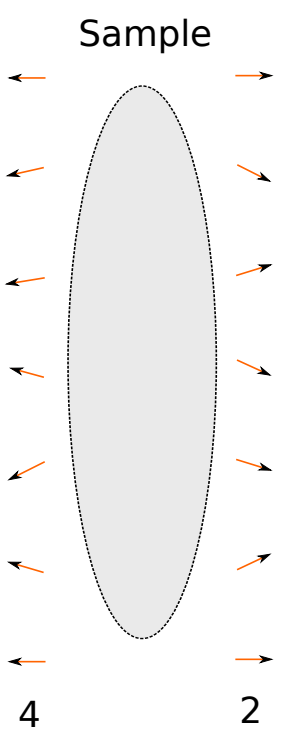

2
EXAMPLE

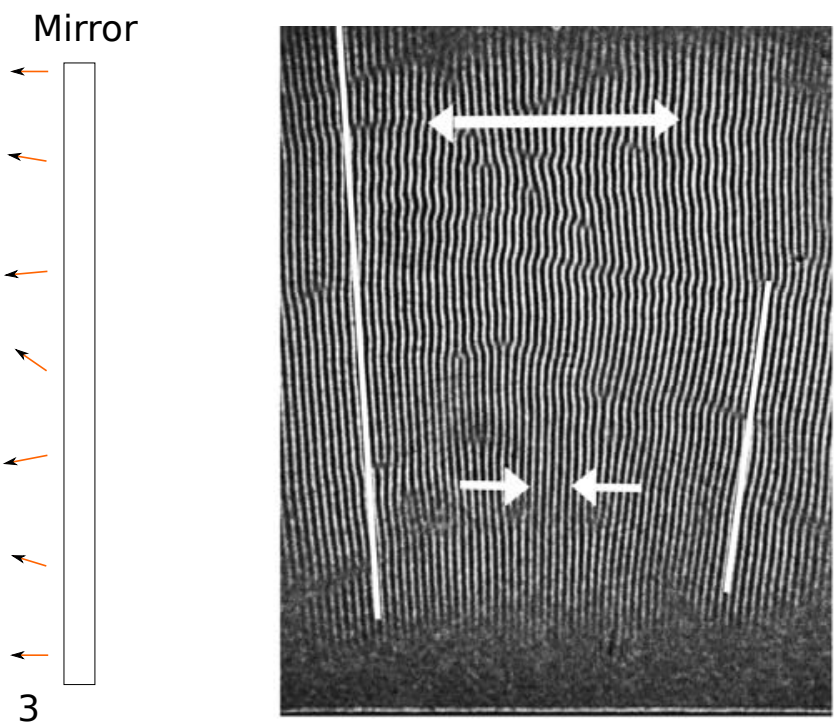

Figure 6.3: LEFT - The principle of the shadowgraph we will use - The shadowgraph sends a beam of parallel light rays $(\mathbf{1})$ represented by the orange arrows. The rays then traverse the sample and are distorted by its irregular interface or its inhomogeneities $(\mathbf{2})$. The light is reflected by the mirror (3) and traverses the sample again (4) to finally be measured by the shadowgraph, which uses some optics algorithm to reverse-engineer the properties of the sample. RIGHT - An example of a shadowgraph output, where we can see the fluctuation in the light intensity. This shows convection patterns. Source: 73

through the said medium, see Fig6.3. If the medium is homogeneous, but has a non-flat interface with the outer propagation medium (and has a different refractive index), there will be some focusing of the light beam (caustics) that can be measured and interpreted by a computer. From the shape and intensity of those caustics, it is possible to reverse-engineer the shape of the interface. In our experiment, the medium that the light will travel through will contain several layers. First, the light will go through the fluid layer. Then, it will traverse the bathymetry layer, which will probably be made out of some resin. Ideally, the bathymetry layer has the exact same refractive index as the fluid, because otherwise there will also be some focusing due to the shape of the interface between the bathymetry and the fluid. Finally, the light will be reflected by the mirror at the bottom of the tank and will come back to the shadowgraph through the bathymetry and the fluid.

When this experiment is shown to be functional, we will move to a bigger tank, at the meter scale, to study propagation in extended random bathymetries in order to observe branching.

\subsection{Conclusion}

In this chapter, we presented the theoretical ground upon which we intend to build a miniaturized tsunami experiment that could fit in a small tank. By making simple estimates of the surface tension and the viscosity of such an experiment, we were able to confirm that this idea should be realizable. The success of such an experiment will of course firstly allow us to confirm our theoretical findings about the branching of tsunami waves. Secondly, this could be used in the near future as a way to do tsunami forecasts. Indeed, considering the propagation speed of the waves in the lab experiment, one run of such a scaled-down tsunami event would be a matter of minutes. Assuming that we have a precise enough model of the bathymetry at hand and that the parameters of the tsunamigenic earthquake are known, such an experiment could 
3D VIEW

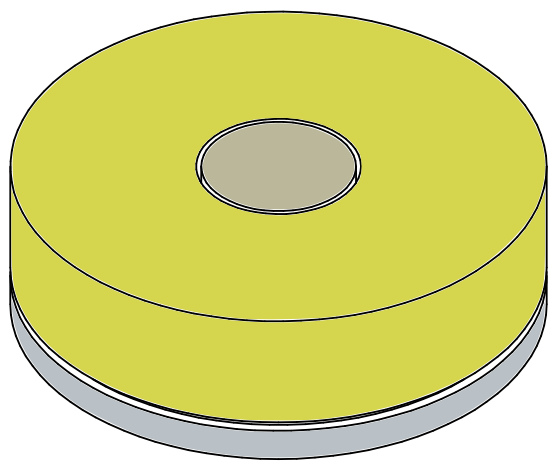

LATERAL CUT

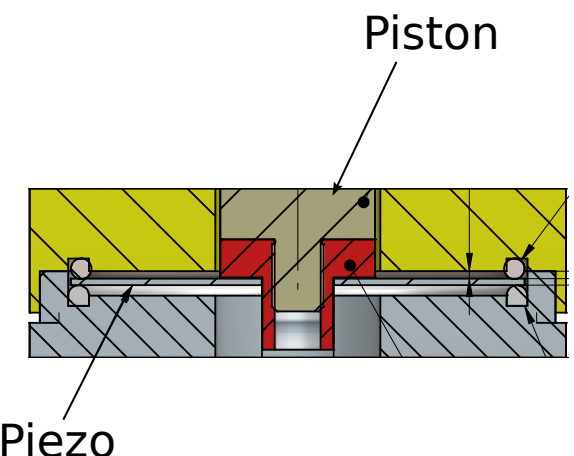

Figure 6.4: On the left is a 3D view of the piston activated by the piezoelectric crystal. On the right, we can see how the piston is constructed. There is a piezoelectric crystal sheet in the center that is embedded in the cylindrical structure (polycarbonate). When it vibrates due to electrical excitations, it makes the piston move. This whole cylindrical device will be embedded in the bottom of the tank. Source: 74
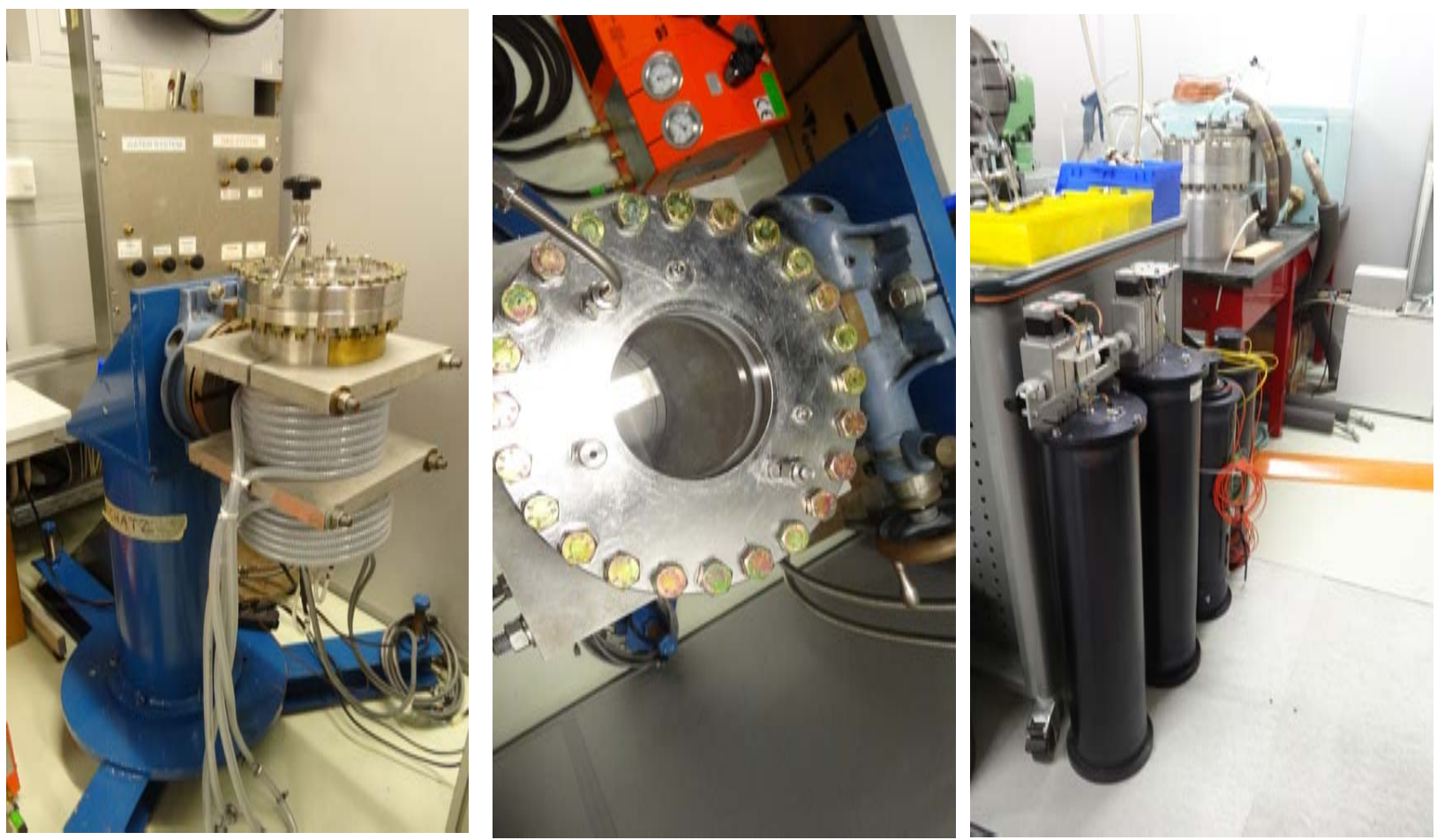

Figure 6.5: Some photographs of the actual material we will use. On the left, we can see the tank in which the experiment will take place (grey cylinder). In the middle, a close up on the interior of the tank, with the window through which the shadowgraph will send and receive the light. Finally, on the left, each of these big black cylindrical boxes is a shadowgraph that can be plugged on top of the tank. 
beat computer simulations for early hazard forecasts. We should, of course, remain cautious, as there is much work to be done before reaching this point.

We are only at the very early stages of this experiment. The idea is to first verify that it works according to our expectation and then work our way to a fully functional experiment. The first step will be to verify how well we can excite the waves with the piezoelectric source. This is currently under investigation by our experimental collaborators. A focusing experiment over a deterministic scatterer will be the second step. We could then compare the experimental data with numerical simulations to see if they sufficiently agree. Once these two tests have been made and passed, we can consider going for a random bathymetry to observe branched flows. 


\section{Chapter 7}

\section{Conclusion and outlook}

In this thesis we have studied the emergence of branched flows in tsunami waves and have developed an appropriate model which allowed us to quantify the mean distance to the first caustics, which is the characteristic length-scale of this phenomenon. We have studied the impact of random focusing on tsunami forecasts and realized that the branching patterns exhibit a high sensitivity to the uncertainties in bathymetry measurements, strongly challenging our current ability to make reliable tsunami forecasts.

Furthermore, we have enriched the theory of two-dimensional branched flows with a study of the effect of more complex correlations, both for classical rays and shallow water waves, showing highly non-trivial dependencies on the relative anisotropies and the length scale ratio. All the results presented in this work are well supported by extensive numerical simulations.

Finally, we proposed an experiment to reproduce tsunami events on laboratory scales. It would allow us to verify our results experimentally. We gave various theoretical estimates which convinced us that realistic tsunami dynamics should indeed be observable in the proposed setup.

Chapter 4 presented the primary results of this thesis. We have shown that tsunamis can be strongly focused by small structures in the bathymetry (i.e. the topography of the ocean floor). First we simulated a tsunami event in the measured bathymetry of the Indian Ocean, in an island-free region where the standard deviation of the bathymetry fluctuations is less than $7 \%$ of the ocean depth. through this we have demonstrated in a realistic model that weak scattering can indeed focus tsunamis to a multitude of their average intensity. We explained these findings by showing that tsunamis are subject to branching of the flow. To this aim, we developed a theory for the emergence of random caustics in shallow water waves. It allowed us to determine the characteristic branching length, which scales with the main parameters of the bathymetry, namely the fluctuation strength and the correlation length. This is surprisingly the same as for Hamiltonian rays, even though the ray equations are very different, as tsunami rays contain a multiplicative noise term unlike classical rays. We have performed extensive numerical simulations in computer generated random bathymetry to support our theoretical results and have shown that shallow water waves indeed follow the predicted scaling behaviour. We found that even bathymetry fluctuations of only $2 \%$ standard deviation lead to focusing on the order of a couple of thousands of kilometers, i.e. scales very relevant for tsunami forecasts. That led us to study what impact branching could have on tsunami forecast. We found that the branching patterns are highly sensitive to the uncertainties in bathymetry measurements, which can be as high as $6 \%$ of the ocean depth. The induced variability in the position of the focused regions therefore reduces considerably our current ability to forecast tsunami events.

In chapter 5, we extended the general theory of branched flow to random media that cannot be described by a correlation function with a single length scale, which is true for many physical systems, especially the ocean floor topography. We studied anisotropic media and media with multi-length-scale correlations and we quantified how the interplay of two different length scales in these random media affect the statistics of random caustics in Hamiltonian systems and in 
tsunami waves. As our results were derived for global anisotropies and very general combinations of correlations, they can be applied to a large variety of systems. We obtained analytical expressions for the characteristic length scale of branching, which exhibit highly non-trivial dependencies on the ratio of the length scales and the propagation direction. For the anisotropic case, we showed analytically that the scaling law for the average distance to the first caustics is the same for classical and tsunami rays, as was already the case in isotropic media (derived in chapter 4) and we confirmed these results with numerical simulations. In the case of media which are isotropic but correlated on more than one length scale, we were able to compute a general scaling law for the same quantity in classical rays, which was confirmed with numerical simulations in the special case of two correlation lengths. Even though we were unable to find the distance to the first caustics analytically for water waves, we numerically showed that they respect the same scaling as classical rays in the case of two correlation lengths.

In chapter 6 , we developed theoretical arguments to support our project of testing our results on tsunami focusing in a laboratory experiment by computing the impact of surface tension and viscosity on the dispersion relation of the linear shallow water wave equation. We showed that, to a reasonably good approximation, the propagation of surface waves in sulfur hexafluoride $S F_{6}$ at high pressure should exhibit the same behavior as tsunami waves in the ocean, with the advantage that it could be observed below the meter scale.

In this work, we built a theory for branching in tsunami waves and showed the importance of this effect in tsunami studies. This opens a wide field of further investigations to improve our understanding of this phenomenon. First of all, a systematic study on how small bathymetry fluctuations on top of larger bathymetry fluctuations can influence the focusing patterns to quantify the reliability of prediction is needed.

Also, the analytical results we derived here were mainly related to the mean distance to the first caustics. This quantity is of great importance in the theory of branched flow, as is gives the characteristic scale of the phenomenon and it also appears in other quantities, such as the average density of branches as a function of the propagation distance from the source, which was computed for classical rays in [37]. It could be interesting to see if such a result can also be computed for tsunami rays. Other interesting statistical quantities, such as the overall amplitude distribution, could also be investigated for tsunamis.

In chapter 3 , we introduced the shallow water equations, which are the common model for tsunami propagation in deep ocean conditions. We decided to neglect the Coriolis force, as it is very weak and not fluctuating. This approximation is generally accepted in the tsunami literature. On the other hand, neglecting the curvature of the Earth can be much more problematic. Indeed, the error on the estimate of lengths grows with distances, meaning that a correction term should be considered when dealing with very long distance propagation $(>2000 \mathrm{~km})$. It would be interesting to include the curvature in our calculation, either as a simple correction factor on the distances, or by trying to derive ray equations from the shallow water wave equations in spherical coordinates, which can be found e.g. in 75.

As this work studied the focusing of the tsunami waves far from the coasts, in the linear regime, it would of course also be of interest to extend the theory to nonlinear waves, where the interplay between the focusing we describe in this thesis and the nonlinearities could only improve our understanding of the run-up.

The theory we developed for anisotropic media has a large range of applicability, e.g. for the transport properties of semiconductors with anisotropic crystals structures or the dynamics of sound wave in anisotropic turbulent flows.

As we already mentioned, branched flow is a universal phenomenon that was studied in many systems over a large range of magnitudes in size, from the micrometer scale in quantum systems to the megameter scale in tsunami waves. In the frame of geophysics, we believe that such a theory could be successfully applied to seismic waves, which would be a good motivation for the study of three-dimensional systems. On a more imaginative note, gravitational waves might also be subject to branching when scattered by massive clouds and/or clusters, the focusing 
properties of which might be exploited to improve the possibilities of measurements.

With this work, we believe we have contributed to the general theory of branched flow and to the improvement of tsunami physics. We hope that our study will help the tsunami community to improve their forecasting abilities and encourage scientists outside this community to join the effort to improve our understanding of this phenomenon. 



\section{Appendix A}

\section{Modeling the random potentials}

Throughout this thesis, we study the effect of random focusing on wave and particle flows in the frame of branched flow. Most of our analytical results are supported by extensive numerical simulations, which most of the time require computer generated random potentials or bathymetries. The purpose of this appendix is to show how we generated them.

The general model used for those random fields is a two dimensional Gaussian random field with a correlation function. The constraints that are common to every case is the fact that we want to be able to model the correlation function $c(x, y)$ by

$$
c(x, y)=\left\langle V\left(x^{\prime}, y^{\prime}\right) V\left(x^{\prime}+x, y^{\prime}+y\right)\right\rangle=\epsilon^{2} f(x, y),
$$

with $f(0,0)=1$ and $\epsilon$ is therefore the effective standard deviation of the Gaussian point process at any location $(x, y)$. Depending on the type of study we make, the potential can have more than one correlation length, as in chapter 5 . We require $c(x, y)$ to be up to four times differentiable in some cases and its Fourier transform has to be non-negative in all cases, as the Wiener-Khinchin theorem states that the Fourier transform of the autocorrelation of a field corresponds to the power spectrum of said field, which cannot be negative.
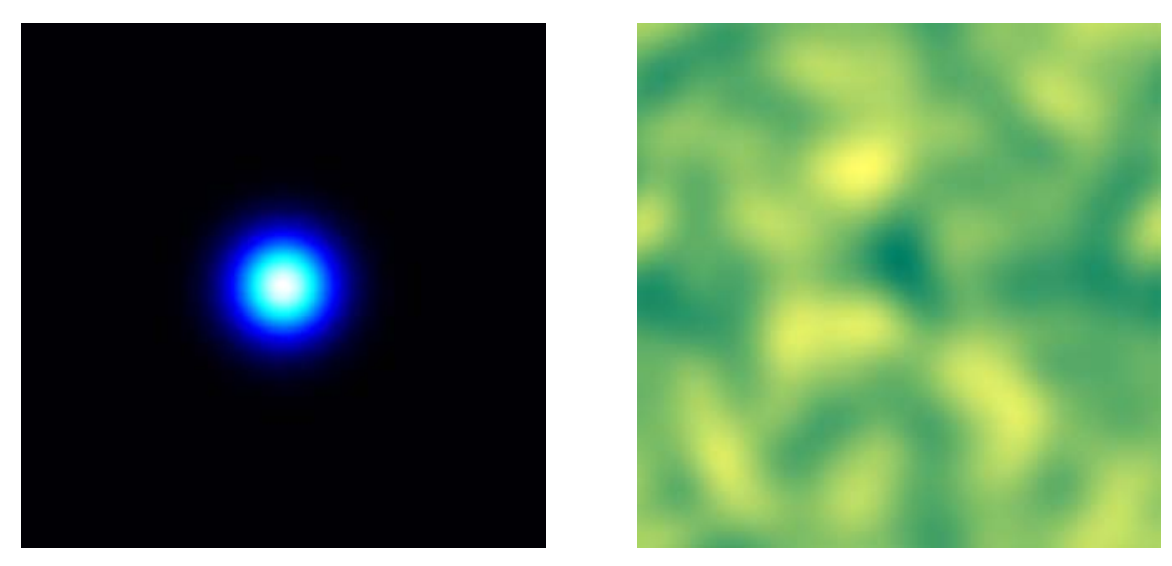

Figure A.1: On the left panel, we can see a typical two dimensional isotropic correlation function (here, a Gaussian), and on the right panel, a potential generated from this correlation function using the methods explained in Eq. A.2.

The random field is constructed in the following way: we start by taking the Fourier transform of the correlation function and take its square root. We then multiply by phases $e^{2 i \pi \varphi(x, y)}$, with $\varphi(x, y)$ distributed uniformly between $[0,1)$. Finally, we Fourier transform back into real space. Mathematically, it can be written 


$$
V(x, y)=\mathcal{F} \mathcal{T}^{-1}\left[\sqrt{\mathcal{F} \mathcal{T}[c(x, y)]} e^{2 \pi i \varphi(x, y)}\right],
$$

where $\mathcal{F} \mathcal{T}$ denotes the Fourier transform. This procedure produces a Gaussian random field $V(x, y)$ because of the central limit theorem, as the Fourier transform is here a sum of independent random variables. The result of this process is illustrated in Fig. A.1.

Numerically, we calculate the potential on a grid and points in-between are interpolated using 2D splines (code developed in $[76]$ ). 


\section{Appendix B}

\section{The Fokker-Planck equation}

In this appendix, we will show the derivation of the Fokker-Planck equation starting from a general stochastic differential equation, as this equation is central in our stochastic analysis. This presentation will be very similar to the one in [37, as it serves the same purpose. More classical references are [55,77].

Let us consider a stochastic dynamical system $\xi(t)$ (a generalization of the results to vectors will be given later) which satisfies

$$
\dot{\xi}(t)=h(\xi, t)+g(\xi, t) \Gamma(t),
$$

where $h(\xi, t)$ and $g(\xi, t)$ are deterministic and $\Gamma(t)$ is a Gaussian white noise with

$$
\left\langle\Gamma(t) \Gamma\left(t^{\prime}\right)\right\rangle=2 \delta\left(t-t^{\prime}\right) .
$$

The goal is to calculate the probability density $P(x, t)$ based on the initial probability density $P\left(x_{0}, t_{0}\right)$ and Eq.B.1. We can write

$$
P(x, t+\tau)=\int T\left(x, t+\tau \mid x^{\prime}, t\right) P\left(x^{\prime}, t\right) \mathrm{d} x^{\prime},
$$

where $T\left(x, t+\tau \mid x^{\prime}, t\right)$ is the transition probability from $\left(x^{\prime}, t\right)$ to $(x, t+\tau)$, with $\tau>0$. As the integral sums over all possible starting points $x^{\prime}$, this equation corresponds to the conservation of probability. We can obtain a differential equation for $P(x, t)$ by Taylor-expanding the integrand of the previous equation in small spatial deviations $\Delta x=x-x^{\prime}$, such that we have

$$
\begin{aligned}
T\left(x, t+\tau \mid x^{\prime}, t\right) P\left(x^{\prime}, t\right) & =T(x-\Delta x+\Delta x, t+\tau \mid x-\Delta x, t) P(x-\Delta x, t) \\
& =\sum_{n=0}^{\infty} \frac{(-1)^{n}}{n !}(\Delta x)^{n} \frac{\partial^{n}}{\partial x^{n}} P(x+\Delta x, t+\tau \mid x, t) P(x, t) .
\end{aligned}
$$

This expression can be used in Eq.(B.3), which then yields

$$
P(x, t+\tau)=\int \sum_{n=0}^{\infty} \frac{(-1)^{n}}{n !}(\Delta x)^{n} \frac{\partial^{n}}{\partial x^{n}} P(x+\Delta x, t+\tau \mid x, t) P(x, t) \mathrm{d} x^{\prime} .
$$

Using the moments $M_{n}(n \geq 1)$ defined by

$$
M_{n}\left(x^{\prime}, t, \tau\right)=\left.\left\langle[\xi(t+\tau)-\xi(t)]^{n}\right\rangle\right|_{\xi(t)=x^{\prime}}=\int\left(x-x^{\prime}\right)^{n} T\left(x, t+\tau \mid x^{\prime}, t\right) \mathrm{d} x,
$$

we can write the time variation as

$$
\begin{aligned}
\frac{P(x, t+\tau)-P(x, t)}{\tau} & =\frac{1}{\tau} \int \sum_{n=1}^{\infty} \frac{(-1)^{n}}{n !}(\Delta x)^{n} \frac{\partial^{n}}{\partial x^{n}} P(x+\Delta x, t+\tau \mid x, t) P(x, t) \mathrm{d} x^{\prime} \\
& =\frac{1}{\tau} \sum_{n=1}^{\infty} \frac{(-1)^{n}}{n !} \frac{\partial^{n}}{\partial x^{n}} M_{n}(x, t, \tau) P(x, t) .
\end{aligned}
$$


We can also Taylor-expand the moments to first order, as we want to go to the limit $\tau \longrightarrow 0$. It yields

$$
\frac{M_{n}(x, t, \tau)}{n !}=D^{(n)}(x, t) \tau+\mathcal{O}\left(\tau^{2}\right)
$$

which can be used to write

$$
\frac{\partial}{\partial t} P(x, t)=\lim _{\tau \longrightarrow 0} \frac{P(x, t+\tau)-P(x, t)}{\tau}=\sum_{n=1}^{\infty}(-1)^{n} \frac{\partial^{n}}{\partial x^{n}} D^{(n)}(x, t) P(x, t) .
$$

In our current case of $\delta$-correlated noise (white noise), the moments for $n>2$ vanish and so we obtain

$$
\frac{\partial}{\partial t} P(x, t)=\left[-\frac{\partial}{\partial x} D^{(1)}(x, t)+\frac{\partial^{2}}{\partial x^{2}} D^{(2)}(x, t)\right] P(x, t),
$$

the well-known as the Fokker-Planck equation.

For the extension to higher dimensions, we will give the F-P equation for the probability $P\left(\vec{x}, t \mid \vec{x}_{0}, t_{0}\right)$ with initial condition

$$
P\left(\vec{x}, t_{0} \mid \vec{x}_{0}, t_{0}\right)=\delta^{n}\left(\vec{x}-\vec{x}^{\prime}\right) .
$$

For this, let us consider a vector equation similar to Eq. B.1] (summation convention implied)

$$
\dot{\xi}_{i}(t)=h_{i}(\vec{\xi}, t)+g_{i j}(\vec{\xi}, t) \Gamma_{j}(t),
$$

containing now multiple white noise with

$$
\left\langle\Gamma_{i}(t) \Gamma_{j}\left(t^{\prime}\right)\right\rangle=2 \delta_{i j} \delta\left(t-t^{\prime}\right) .
$$

The associated F-P equation is then given by 55,77

$$
\frac{\partial}{\partial t} P\left(\vec{x}, t \mid \vec{x}_{0}, t_{0}\right)=\left[-\frac{\partial}{\partial x_{i}} D_{i}^{(1)}(\vec{x}, t)+\frac{\partial^{2}}{\partial x_{j} \partial x_{j}} D_{i j}^{(2)}(\vec{x}, t)\right] P\left(\vec{x}, t \mid \vec{x}_{0}, t_{0}\right),
$$

with the drift and diffusion coefficients generally given by

$$
\begin{aligned}
D_{i}^{(1)}(\vec{x}, t) & =h_{i}(\vec{x}, t)+g_{k j}(\vec{x}, t) \frac{\partial}{\partial x_{k}} g_{i j}(\vec{x}, t), \\
D_{i j}^{(2)}(\vec{x}, t) & =g_{i} k(\vec{x}, t) g_{j k}(\vec{x}, t) .
\end{aligned}
$$

It is also worth mentioning that the F-P equation can be understood as a conservation law for the probability current $J$ defined by (in on dimension)

$$
J=\left[D^{(1)}(x, t)-\frac{\partial}{\partial x} D^{(2)}(x, t)\right] P(x, t),
$$

such that we can write

$$
\frac{\partial}{\partial t} P+\frac{\partial}{\partial x} J=0 .
$$

Finally, we also need to mention that there is a backward F-P equation in opposition to the forward equation presented earlier. The forward equation describes the time evolution of the probability density $\mathrm{P}$, starting from a set of initial condition $\left(x_{0}, t_{0}\right)$, for later times $t>t_{0}$. In some situations, however, one would like to know how the initial distribution depends on fixed final conditions, as it happens to be the case for the mean first passage time calculation used in chapter 2. For such cases, on can write a reverse time evolution equation with a backward F-P equation which is given by

$$
\frac{\partial}{\partial t_{0}} P\left(\vec{x}, t \mid \vec{x}_{0}, t_{0}\right)=\left[-D_{i}^{(1)}\left(\vec{x}_{0}, t_{0}\right) \frac{\partial}{\partial x_{i}^{0}}+D_{i j}^{(2)}\left(\vec{x}_{0}, t_{0}\right) \frac{\partial^{2}}{\partial x_{j}^{0} \partial x_{j}^{0}}\right] P\left(\vec{x}, t \mid \vec{x}_{0}, t_{0}\right),
$$

associated with the final condition

$$
P\left(\vec{x}, t \mid \vec{x}_{0}, t\right)=\delta^{n}\left(\vec{x}-\vec{x}^{\prime}\right) .
$$




\section{Appendix C}

\section{Modeling a Fault source}

At the end of chapter 4, we needed to model a tsunami source that had a geometry closer to what we would find in the case of a dip-slip earthquake, which usually occurs on a fault. This topic has been studied in [78] and they propose a method that allows one to transpose the deformation of the ocean floor onto the surface of the ocean. As we used this kind of initial condition throughout this thesis, we will apply this method here, using the geometry of the source that they suggest. Considering that the fault's main axis is in the $y$-direction, i.e. the main propagation direction of the wave will be $x$, the complete expression we used is

$$
\begin{aligned}
\eta(x, y, t=0) & =\eta_{0}\left[\arctan \left(\frac{x}{d}\right)-\arctan \left(\frac{x}{d-L}\right)-\frac{(d-L) x}{x^{2}+(d-L)^{2}}+\frac{d x}{x^{2}+d^{2}}\right] \\
& \times\left[1+\tanh \left(\frac{y-D}{a}\right) \tanh \left(\frac{-D-y}{a}\right)\right] .
\end{aligned}
$$

where $\eta_{0}$ gives the initial amplitude of the wave, $d$ and $L$ are the parameters of the fault (as described in [78]) (here $\mathrm{d}=4$ and $\mathrm{L}=2$ ), $D$ is half of the length of the fault (here 400) and $a$ is steepness factor for the edge of the fault (here $\mathrm{a}=20$ ). The first brackets contain the fault function described in 78, as shown in Fig C.1. The second bracket is a combination of smooth step functions (hyperbolic tangents) which define the length of the fault. A 3d view of this initial condition can be seen in Fig C.2.

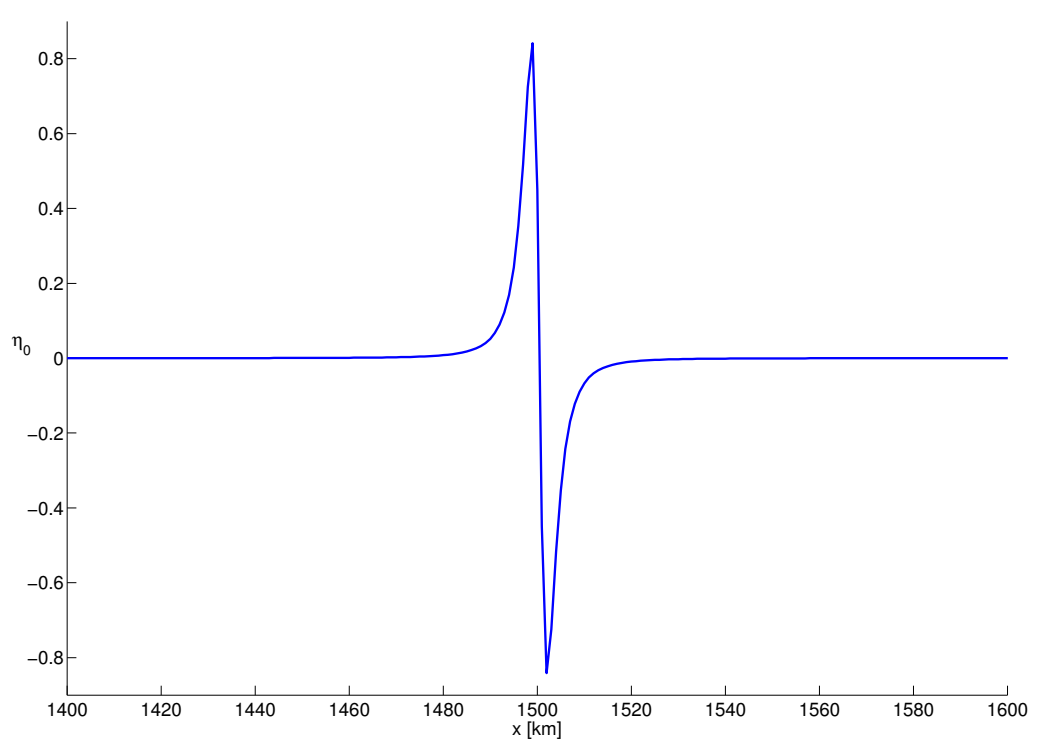

Figure C.1: The profile of the fault function. 


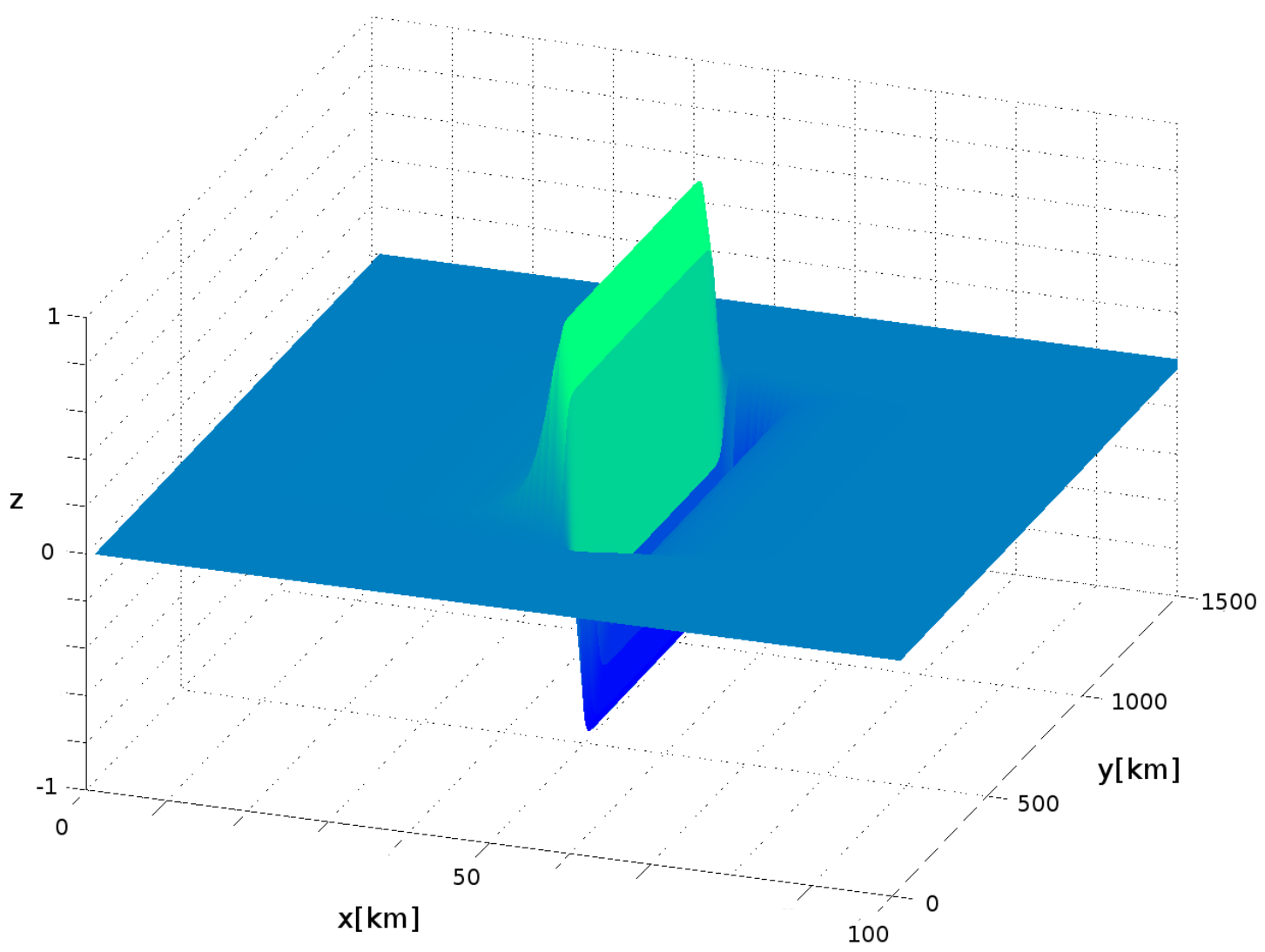

Figure C.2: A 3D view of the fault initial condition that we used. The units in $z$ are arbitrary 


\section{Appendix D}

\section{Hamiltonian for shallow water rays}

When we needed to derive the equations for the time evolution of the stability matrix elements for the shallow water rays in chapter 4, our first idea was to find a Hamiltonian and follow what we did in chapter 2. Furthermore, we could have used this Hamiltonian in order to study the evolution of the curvature of the action through the Hamilton-Jacobi equation, which would have probably made most of our derivations much easier. This, however, appears impossible with the variables $(\vec{x}, \vec{p})$, as we would be looking for a Hamiltonian function $\mathcal{H}(\vec{x}, \vec{q})$, such that

$$
\begin{aligned}
\dot{\vec{x}} & =\frac{\partial \mathcal{H}}{\partial \vec{p}}, \\
\dot{\vec{p}} & =-\frac{\partial \mathcal{H}}{\partial \vec{x}} .
\end{aligned}
$$

This can be transformed into a set of PDEs, using the known ray equations derived in chapter 3 ,

$$
\begin{aligned}
\frac{\partial \mathcal{H}}{\partial \vec{p}} & =(1-\beta(\vec{x})) \vec{p}, \\
-\frac{\partial \mathcal{H}}{\partial \vec{x}} & =\frac{c_{0}^{2} \nabla \beta(\vec{x})}{2(1-\beta(\vec{x}))} .
\end{aligned}
$$

From the first equation, we could already say that

$$
\mathcal{H}(\vec{x}, \vec{p})=\frac{1}{2}(1-\beta(\vec{x}))(\vec{p} \cdot \vec{p})+F(\vec{x}),
$$

where $F(\vec{x})$ is an arbitrary function of $\vec{x}$. This is unfortunately not compatible with the second equation, which leads to

$$
\frac{c_{0}^{2} \nabla \beta(\vec{x})}{2(1-\beta(\vec{x}))}=-\frac{1}{2} \nabla \beta(\vec{x})(\vec{p} \cdot \vec{p})+\nabla F(\vec{x})
$$

which is unsolvable since $F(\vec{x})$ is only a function of $\vec{x}$ and there is a $\vec{p} \cdot \vec{p}$ term left.

Our inability to write the shallow water ray equations in terms of a Hamiltonian has strongly complicated our analysis throughout chapter 4 .

This simple calculation does not exclude, however, that there might exist a pair of canonically conjugated variables $(\vec{\chi}(\vec{x}, \vec{p}), \vec{\psi}(\vec{x}, \vec{p}))$ for which there is a Hamiltonian formulation to this problem. Our attempts at finding such variables failed. 



\section{Appendix E}

\section{Remarks on the numerical simulations and open questions}

Most of the theoretical work presented in this thesis is supported by numerical simulations that confirmed the analytical results. As the numerics are omnipresent in this thesis and actually needed a lot of care, we believe that it is relevant to give some comments on our procedures. Even though the ray simulations were calculated using methods that were sensibly different from the ones needed for the wave simulations, there was a key component that was common to both, which is the random potential. We used the same code for both kinds of simulation and we refer the reader to appendix A for details concerning our model and the relevant references. One of the biggest challenges coming with such a tool is that we are working with a finite size grid, meaning that the correlation functions are truncated at the boundaries. This fact can lead to troubles when dealing with heavy-tailed correlation functions, such as power-laws. Indeed, as a significant part of the correlation function is cut off by the boundaries of the grid, the actual correlation function of the output potential is not the same as the one we input in the code. Typically, once we have a random potential, we can reverse-engineer the correlation function by inverting Eq. A.2 in the following way

$$
c(x, y)=\mathcal{F} \mathcal{T}^{-1}\left[|\mathcal{F} \mathcal{T}(V(x, y))|^{2}\right] .
$$

Fortunately, most of the present work was done using Gaussian correlation functions, which decay sufficiently fast such that we avoid this problem. We have to mention, though, that we had problems when we tried to confirm the correlation function factor $\kappa_{1}^{-1 / 3}$ in the scaling for the distance to the first caustics from Eq.4.60 as even the alternative correlation function $c(r)=\cosh ^{-1}\left(r / l_{c}\right)$ did not give the same result for the prefactors $C_{s}^{w}$, with values differing by $20 \%$. We have so far failed to explain why. Another issue, which is however far less problematic, comes from the fact that enough grid points per correlation length are needed in order to have a high enough resolution of the correlation function. We typically found that a minimum of 12 grid points per correlation length is needed.

On the topic of correlation function, we realized that the expression for $\sigma_{2}$ given in chapter 2 can sometimes be problematic. Indeed, with $\sigma_{2}^{2}$ given by

$$
\sigma_{2}^{2}=\frac{6 \epsilon^{2}}{l_{c}^{3} c_{0}} \kappa_{2}
$$

and $\kappa_{2}$ defined as

$$
\kappa_{2}:=\int_{-\infty}^{+\infty} d z f^{(2)}\left(z^{2}\right)
$$


we sometimes obtain negative values for $\sigma_{2}^{2}$, e.g. for $c(r)=\epsilon^{2} e^{-r^{4} / l_{c}^{4}}$, as we have

$$
\kappa_{2}:=\int_{-\infty}^{+\infty} d z\left[4 z^{4}-2\right] e^{-z^{4}}<0 .
$$

We so far failed to find a proper general constraint on the correlation functions for the diffusion approximation to be valid, but it appears that there are functions that do not allow it. For the classical ray simulations, we used the same code that was used for 37, which we also adapted to the water ray simulations, mainly by changing the ray equations and the stability matrix equations. This code produces potentials of a finite size, which are then repeated in a periodic paving. This can lead to problems when trajectories travel over long distances, as they cover several times the same potential, which can bias the statistic of the first caustics, as we observed many times when we produced the plots.

For the wave simulations, the whole grid consists in a single potential, so we did not have to concern ourselves with this issue. However, another problem is recurrent with the wave simulations, which is the resolution. In order for the waves to be smooth and to behave like waves, we need to have a minimal resolution in the wave profile. Again a minimum of 12 grid points per wavelength appears to be a good choice. Furthermore, in order to work in the geometrical optics limit and easily visualize branching patterns, the correlation length should be sufficiently larger than the wavelength, typically 10 times larger. However, branching and the associated heavy tailed intensity distribution are still present with $\lambda \sim l_{c}$. Finally, considering the above-mentioned problem of the number of correlation lengths per grid and the fact that caustics usually emerge after a few correlation lengths, this constrains us to using grids that have at least $1000 \times 1000$ grid points and sometimes manymore (up to $11000 \times 11000$ for the scaling in the point source case). This explains why we mentioned many times how demanding the wave simulations are. 


\section{Bibliography}

[1] Thorne Lay et al. The great sumatra-andaman earthquake of 26 december 2004. Science, $308,2005$.

[2] A. Kundu. Tsunamis and Nonlinear Waves. Springer, 2007.

[3] http://www.npa.go.jp/archive/keibi/biki/higaijokyo_e.pdf.

[4] http://nctr.pmel.noaa.gov/model.html.

[5] http://nctr.pmel.noaa.gov/Dart/.

[6] M. V. Berry. Focused tsunami waves. Proceedings of the Royal Society A, 463(2087):3055, 2007.

[7] U. Kanoglu, V. V. Titov, B. Aydin, C. Moore, T. S. Stefanakis, H. Zhou, M. Spillane, and C. E. Synolakis. Focusing of long waves with finite crest over constant depth. Proceedings of the Royal Society A: Mathematical, Physical and Engineering Sciences, 469(2153):20130015-20130015, February 2013.

[8] M. A. Topinka, B. J. LeRoy, R. M. Westervelt, S. E. J. Shaw, R. Fleischmann, E. J. Heller, K. D. Maranowski, and A. C. Gossard. Coherent branched flow in a two-dimensional electron gas. Nature, 410(6825):183-186, March 2001.

[9] S. Barkhofen, J. J. Metzger, R. Fleischmann, U. Kuhl, and H.-J. Stöckmann. Experimental observation of a fundamental length scale of waves in random media. Phys. Rev. Lett., 111(18):183902, November 2013.

[10] Jakob Metzger, private communications.

[11] D.R. Solli et al. Optical rogue waves. Nature, 450:1054-1057, 2007.

[12] E. J. Heller, L. Kaplan, and A. Dahlen. Refraction of a Gaussian seaway. Journal of Geophysical Research, 113:C09023, 2008.

[13] L. Lindgren. Some properties of a normal process near a local maximum. Math. Statist., 41:1870-1883, 1970.

[14] M. S. Longuet-Higgins and R. W. Stewart. The changes in amplitude of short gravity waves. J. Fluid Mech., 10:529-549, 1961.

[15] O.M. Phillis, D. Gu, and M. Donelan. Expected structure of extreme waves in a gaussian sea, part i : Theory and swad buoy measurements. J. Phys. Oceanogr., 23:992-1000, 1993.

[16] O.M. Phillis, D. Gu, and M. Donelan. Expected structure of extreme waves in a gaussian sea, part ii : Swade scanning radar altimeter measurements. J. Phys. Oceanogr., 23:2297-2309, 1993.

[17] C. Kharif and E. Pelinovsky. Physical mechanism of the rogue wave phenomenon. European Jounral of Mechanics - B/Fluids, 22:603-634, 2003. 
[18] M. Onorato, A. R. Osborne, M. Serio, and S. Bertone. Freak waves in random oceanic sea states. Phys. Rev. Lett., 86(25):5831-5834, Jun 2001.

[19] M. G. Brown. Space-time surface gravity wave caustics: structurally stable extreme wave events. Wave Motion, 33(2):117 - 143, 2001.

[20] P. Müller, C. Garrett, and A. Osborne. Rogue waves. Oceanography, 18:66-75, 2005.

[21] M. P. Jura, M. A. Topinka, L. Urban, A. Yazdani, H. Shtrikman, L. N. Pfeiffer, K. W. West, and D. Goldhaber-Gordon. Unexpected features of branched flow through high-mobility two-dimensional electron gases. Nat Phys, 3(12):841-845, 2007.

[22] L. Kaplan. Statistics of branched flow in a weak correlated random potential. Phys. Rev. Lett., 89(18):184103, October 2002.

[23] J. J. Metzger, R. Fleischmann, and T. Geisel. Universal statistics of branched flows. Phys. Rev. Lett., 105(2):020601, July 2010.

[24] K. E. Aidala et al. Imaging magnetic focusing of coherent electron waves. Nat Phys, 3(7):464-468, June 2007.

[25] E. Jakeman, E.R. Pike, and P.N. Pusey. Photon correlation study of stellar scintillation. Nature, 1976.

[26] M.V. Berry. Focusing and twinkling: critical exponents from catastrophes in non gaussian random short waves. J. Phys A, 1977.

[27] Y. Kravtsov and Y. Orlov. Geometrical optimics of Inhomogeneous media. Spinger, 1990.

[28] Y. Kravtsov. Propagation of electromagnetic waves through a turbulent atmosphere. Reports on progress in physics, 1992.

[29] E.M. Salomon. Caustic diffraction fields in a downward refracting atmosphere. The Journal of the Acoustical Society of America, 104:3259-3272, 1998.

[30] M.A. Wolfson and F.D. Tappert. Study of horizontal multipaths and ray chaos due to ocean mesoscale structure. J. Acoust. Soc. Am., 107(1):154-162, 2000.

[31] M. A. Wolfson and S. Tomsovic. On the stability of long-range sound propagation through a structured ocean. J. Acoust. Soc. Am., 109(6):2693, 2001.

[32] V. E. Ostashev. Sound propagation and scattering in media with random inhomogeneities of sound speed, density and medium velocity. Waves in Random Media, 4(4):403-428, 1994.

[33] P. Blanc-Benon, D. Juvé, V. E. Ostashev, and R. Wandelt. On the appearance of caustics for plane sound-wave propagation in moving random media. Waves in Random Media, 5(2):183-199, 1995.

[34] RA Vadov. Observation and location of caustics in the ocean at different sound frequencies. Acoustical Physics, 43(4):401-405, JUL-AUG 1997.

[35] M. G. Brown, J. A. Colosi, Tomsovic S., A. L. Virovlyansky, M. A. Wolfson, and G.M. Zaslavsky. Ray dynamics in long-range deep ocean sound propagation. The Journal of the Acoustical Society of America, 113(5):2533-2547, 2003.

[36] E. Z. Gribova. Statistics of caustics in an underwater sound channel. Acoustical Physics, 53(6):730-735, NOV 2007. 
[37] J. J. Metzger. Branched Flow and Caustics in Two-Dimensional Random Potentials and Magnetic Fields. PhD thesis, Niedersächsische Staats-und Universitätsbibliothek Göttingen, 2010.

[38] M. V. Berry and C. Upstill. Catastrophe optics: morphologies of caustics and their diffraction patterns. Prog. Opt, 18:257-346, 1980.

[39] V. I. Arnold. Catastrophe theory. Springer, 2004.

[40] R. Courant and D. Hilbert. Methods of Mathematical Physics, volume 2. Wiley-Interscience, 1962.

[41] G. B. Whitham. Linear and Nonlinear Waves. Wiley \& Sons, Inc., 1974.

[42] Manuel Delgado. The lagrange-charpit method. SIAM Review, 39(2):298-304, 1997.

[43] V.I. Arnold. Lagrangian manifold with singularities, asymptotic rays, and the open swallowtail. Functional Analysis and its Applications, 15:235-246, 1981.

[44] R.G Littlejohn. The van-vleck formula, maslov theory and phase space geometry. J. Stat. Phys., 68:7-50, 1992.

[45] H. Guggeheimer. Differential Geometry. Dover, 1977.

[46] R. Granger. Fluid Mechanics. Dover, 1995.

[47] A. N. Malakhov and A. I. Sachev. Lagrangian and eulerian descriptions of the statistical properties of light waves. Radiofizika, 19:1368-1377, 21976.

[48] V. I. Klyatskin. Stochastic equations through the eye of the physicist. Elsevier, 2005.

[49] V. A. Kulkarny and B.S. White. Focusing of waves in turbulent inhomogeneous media. Phys. Fluids, 25(10):1770, 1982.

[50] R. L Stratonovich. Topics in the theory of random noise., volume 1. Gordon and Breach, 1963.

[51] G. C. Papanicolau and W. Kohler. Asymptotic theory of mixing stochastic ordinary differential equations. Comm. Pure Appl. Math. 27, 641-668, 1974.

[52] D. A. Dawson and G. C. Papanicolau. A random wave process. Appl. Math. Optim.12, 97? 114, 1984.

[53] L. Hesselink and B. Sturtevant. Propagation of weak shocks through a random medium. Journal of Fluid Mechanics 196, 513?553, 1988.

[54] R. J. Adler and J. E. Taylor. Random Fields and Geometry. Springer, 2007.

[55] C. Gardiner. Handbook of Stochastic Methods. Springer, 2004.

[56] W. Feller. The parabolic differential equation and the associated semi-groups of transformations. Ann. Math., 55, 1952.

[57] V. I. Klyatskin. Caustics in random media. Waves in Random Media, 3, 1993.

[58] S. N Ward and S. Day. Tsunami thoughts. Recorder J. Can. Soc, 2005.

[59] R. Shankar. Retracing Tsunami Rays, chapter 4. Springer, 2007.

[60] D. L. George. Finite Volume Methods and Adaptive Refinement for Tsunami Propagation and Inundation. PhD thesis, University of Washington, 2006. 
[61] Johnson R. A Modern Introduction to the Mathematical Theory of Water Waves. Cambridge University Press, 1997.

[62] M.S. Longuet-Higgins. On the statistical distribution of the heights of sea waves. J. Mar. Res., 237:1245-1266, 1952.

[63] J.-S. Gagnon, S. Lovejoy, and D. Schertzer. Multifractal earth topography. Nonlinear Process in Geophysics, EGU, 13(5):541-570, 2006.

[64] http://www.gebco.net/.

[65] T Kramer, E. J. Heller, and R. E. Parrott. An efficient and accurate method to obtain the energy-dependent green's function for general potentials. Journal of Physics, Conference Series, 99, 2008.

[66] D. Dutykh and F. Dias. Energy of tsunami waves generated by bottom motion. Proceedings of the Royal Society A: Mathematical, Physical and Engineering Science, 465(2103):725-744, 2009 .

[67] Pengzhi Lin. Numerical modeling of water waves. Taylor and Francis, 2008.

[68] D.T. Sandwell, S.T. Gille, and W.H.F. Smith. Bathymetry from space: Oceanography, geophysics, and climate, geoscience professional services, bethesda, maryland, June 2002.

[69] J. J. Becker, D. T. Sandwell, W. H. F. Smith, J. Braud, B. Binder, J. Depner, J. D. Fabre, J. Factor, S. Ingalls, S.-H. Kim, R. Ladner, K. Marks, S. Nelson, A. Pharaoh, R. Trimmer, J. Von Rosenberg, G. Wallace, and P. Weatherall. Global bathymetry and elevation data at 30 arc seconds resolution: Srtm30 plus. Marine Geodesy, 2009.

[70] Alexei Krekhov, private communications.

[71] H Lamb. Hydrodynamics. Cambridge University Press, 1977.

[72] G.-Q. Chen and M. Perepelitsa. Shallow water equations : viscous solution and inviscid limit. The Journal of Applied Mathematics and Physics, 2011.

[73] www.lfpn.ds.mpg.de/patternform/documents/Plapp_PhD/chapt1_ps.gz.

[74] Prasanth Prabhakran, private communications.

[75] B. Levin and M. Nosov. Physics of tsunamis. Springer, Dordrecht; London, 2009.

[76] K. Broeking. Transport in micro-hallbars. Master's thesis, Georg-August University, Goettingen, 2006.

[77] H. Risken. The Fokker-Planck equation. Springer, 1996.

[78] D. Dutykh and F. Dias. Tsunami generation by dynamic displacement of sea bed due to dip-slip faulting. Mathematics and Computers Simulation, 80:837-848, 2009. 


\section{Acknowledgment}

I wish to thank Theo Geisel for offering me the opportunity to work in such a great environment. The institute wouldn't be such a nice place to work at without him. He is also the man behind the fantastic winter seminars, which every year proposed a very rich experience, both on the scientific and human level. I also want to thank him for giving me the opportunity to attend so many conferences, which not only enriched my scientific knowledge, but also gave me the chance to discover new countries and meet great people.

I would like to thank Ragnar Fleischmann for supervising me throughout my PhD studies. He was always available when I needed some guidance and his patience and optimism are admirable. He taught me countless things which, I believe, made me a better scientist. I also want to thank him for always accepting my numerous requests to work from abroad, which allowed me to spend more time with my family and friends.

I want to thank Jakob Metzger for always being there for me when I was lost. He helped me to approach a field that was completely new to me and was never short of time to guide me through the steps of what is now my thesis and to teach me how things are done, particularly when it came to programming skills (or my lack thereof). We also had great times at the various conferences we attended together, as well as during some more extracurricular activities. I want to thank Eberhard Bodenschatz for his many valuable suggestions, including discussions about the ocean's bathymetry database and its use in our numerical simulations, as well as the idea of the experiment described in chapter 6 .

I thank Prasanth Prabhakaran, Alexei Krekhov and Holger Nobach for accepting to work on our rather ambitious experiment. We had fruitful discussions about the realization of such a project. A special thank to Prasanth, who took some time to show me the laboratory and explain to me the various parts of the experimental setup.

I thank Erik Schultheis for working with me on the problem of three-dimensional rays in anisotropic potentials. Even though we have not yet succeeded, he brought very good inputs to this current research and his simulations and plots were very useful.

More generally, I want to thank all my collaborators and colleagues for contributing to the very nice working environment at the institute. We also had a lot of good times during the winter seminars and other extracurricular activities. A special thank to Stephan Eule for making my times at the APS conferences a lot funnier, and also for his very frank way of speaking, which often led me to improve my approach to my work. Another special thanks to Türeiti Keith, who proofread this thesis and made it more readable.

I also want to thank the people of the HPC and IT departments, namely Denny Fliegener, Yorck-Fabian Beensen and Barbara Guichemer. Other than just making the HPC work, Denny also helped me several times on optimizing my simulations on the cluster and most of the numerical results presented in this thesis wouldn't exist without him. Yorck and Barbara always provided me the needed assistance with every IT matters.

I also want to thank the people of the administration, especially Ayse Bolik, Vika Novak and Regina Wunderlich. They helped me countless times to find my way in the various administrative tasks, but also more generally advised me on some more practial matters for my life in Göttingen. The same goes for the people at the IMPRS office, namely Frauke Bergmann, Antje Erdmann and Tina Trost. 
Finally, I want to thank my family and close friends for supporting me throughout my time in Göttingen by finding time for me, either by coming to visit me here or by being available whenever I was. It helped me more than they can imagine. 


\section{Curriculum Vitae}

Henri-Philippe Degueldre, MSc in Physics

Born on August 30, 1988, in Courbevoie, France

Citizenship: German

$\underline{\text { Time and location }}$

Occupation

08.1994-07.2003 Delémont $(\mathrm{CH})$

Elementary and secondary school

08.2003-07.2006 Porrentruy $(\mathrm{CH})$

High School

10.2006-07.2009 Lausanne $(\mathrm{CH})$

Bachelor in Physics, EPFL

09.2009-07.2010 Lausanne (CH)

Master in Theoretical Physics, EPFL

08.2010

Gainesville, FL (US)

$-12.2010$

Master's Project on "One-loop field strengths of charges and dipoles on a locally deSitter background", UF

01.2011-07.2011 Lausanne (CH)

Sabbatical

08.2011-12.2011 Morges (CH)

Financial Consultant (FCG Group)

01.2012-07.2015 Goettingen (D)

$\mathrm{PhD}$ student at MPIDS 